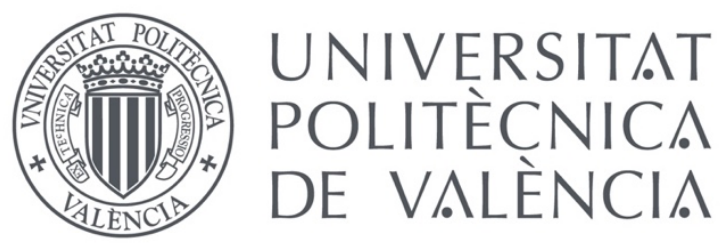

TESIS DOCTORAL

\title{
LOS VERBOS MODALES EN LAS INTRODUCCIONES Y EN LAS \\ CONCLUSIONES DE LOS ARTÍCULOS CIENTÍFICOS DE TURISMO
}

PRESENTADA POR:

María Elena Domínguez Morales

DIRIGIDA POR:

Dr. Francisco José Álvarez Gil

Dra. María Luisa Carrió Pastor

JUNIO 2021 



\section{Resumen}

El artículo de investigación en el campo de los estudios de turismo no ha sido suficientemente estudiado, a pesar de ser una disciplina que representa un sector de desarrollo económico para numerosos países. Otros géneros textuales relacionados con el registro turístico como el resumen, los folletos y las páginas webs en el registro turístico, los últimos con gran relevancia por su claro impacto social y económico de los estudios turísticos han recibido mayor atención. La investigación científica en turismo, sin embargo, provee a las organizaciones, ya sean públicas o privadas, de información relevante para la mejora de la actividad que se traducen en recomendaciones que, con frecuencia, se aportan en las conclusiones de estos trabajos. Se entiende, por lo tanto, que existe una relación directa entre el progreso del sector y la investigación empírica, lo que, a su vez, indica el interés por la publicación académica periódica. Por este motivo, en este trabajo, se incluye una caracterización formal del género en una estructura de secciones. Este estudio se complementa con un análisis de las estructuras léxicogramaticales recurrentes en cada una de las secciones del artículo científico y las variables léxicas y sintácticas que caracterizan al texto dentro del registro científico, tanto por la complejidad como por el nivel de elaboración que exhibe la lengua usada en estos trabajos. Estas estructuras persiguen indicar la perspectiva de los autores, entre otros aspectos.

Dicho esto, el objetivo de este trabajo es analizar las perífrasis modales, sus significados y la existencia de variación en las secciones mencionadas del artículo científico en un corpus de las secciones denominadas "introducción" y "conclusión" del artículo de investigación en la disciplina de turismo. Estos textos se han extraído de revistas en el campo y con un índice de impacto notorio en la especialidad. Las perífrasis modales reflejan el punto de vista del hablante. Como parte de la metodología, se usan herramientas de lingüística de corpus que permiten la gestión de los textos y su consulta, además de la extracción de ejemplos concretos que ilustren las formas, las categorías semánticas y las funciones que se encuentren en los textos estudiados. Sin embargo, dado que el contexto es fundamental para poder identificar los significados modales, parte del análisis de los textos requiere de un reconocimiento visual directo de cada caso, pues una misma perífrasis modal puede indicar diferentes significados. El análisis y discusión de los datos se nutre de estudios en lingüística funcional que permitan dar cuenta de los usos y funciones 
Las conclusiones indican que, en efecto, existe variación no solo en cuanto a las formas usadas en cada una de las introducciones y en las conclusiones, sino también con respecto al significado. En este sentido, se constata que la modalidad dinámica es más frecuente en las introducciones, según frecuencias relativas, y la modalidad epistémica es más frecuente en las conclusiones. Igualmente, se han detectado varias funciones estrechamente relacionadas con los significados modales. Así, la función de mitigación y cortesía lingüística se relaciona en mayor medida con la modalidad epistémica y lo mismo ocurre con la formulación del significado inferencial, que refiere a la necesidad epistémica. La expresión de la factualidad y de la imposibilidad se nutren de la modalidad dinámica, mientras que la predicción, la organización de los contenidos, la autoridad y la recomendación se manifiestan con el uso de la modalidad deóntica.

La tesis se estructura en cinco capítulos y las referencias bibliográficas que se incluyen al final de este trabajo. 


\section{Resum}

L'article d'investigació en el camp dels estudis de turisme no ha sigut prou estudiat, malgrat ser una disciplina que representa un sector de desenvolupament econòmic per a nombrosos països. Altres gèneres textuals relacionats amb el registre turístic com el resum, els fullets i les pàgines webs en el registre turístic, els últims amb gran rellevància pel seu clar impacte social i econòmic dels estudis turístics, han rebut major atenció. La investigació científica en turisme, no obstant això, proveeix a les organitzacions, ja siguen públiques o privades, d'informació rellevant per a la millora de l'activitat que es tradueixen en recomanacions que, amb freqüència, s'aporten en les conclusions d'aquests treballs. S'entén, per tant, que existeix una relació directa entre el progrés del sector i la investigació empírica, la qual cosa, al seu torn, indica l'interés per la publicació acadèmica periòdica. Per aquest motiu, en aquest treball, s'inclou una caracterització formal del gènere en una estructura de seccions. Aquest estudi es complementa amb una anàlisi de les estructures lèxic-gramaticals recurrents en cadascuna de les seccions de l'article científic i les variables lèxiques i sintàctiques que caracteritzen al text dins del registre científic, tant per la complexitat com pel nivell d'elaboració que exhibeix la llengua usada en aquests treballs. Aquestes estructures persegueixen indicar la perspectiva dels autors, entre altres aspectes.

Dit això, l'objectiu d'aquest treball és analitzar les perífrasis modals, els seus significats i l'existència de variació en les seccions denominades "introducció" i "conclusió" de l'article d'investigació en la disciplina de turisme. Aquests textos s'han extret de revistes en el camp i amb un índex d'impacte notori en l'especialitat. Les perífrasis modals reflecteixen el punt de vista del parlant. Com a part de la metodologia, s'usen eines de lingüística de corpus que permeten la gestió dels textos i la seua consulta, a més de l'extracció d'exemples concrets que il-lustren les formes, les categories semàntiques i les funcions que es troben en els textos estudiats. No obstant això, atés que el context és fonamental per a poder identificar els significats modals, part de l'anàlisi dels textos requereix d'un reconeixement visual directe de cada cas, perquè una mateixa perífrasi modal pot indicar diferents significats. L'anàlisi i discussió de les dades es nodreix d'estudis en lingüística funcional que permeten donar compte dels usos i funcions

Les conclusions indiquen que, en efecte, existeix variació no sols quant a les formes usades en cadascuna de les introduccions i en les conclusions, sinó també respecte al 
significat. En aquest sentit, es constata que la modalitat dinàmica és més freqüent en les introduccions, segons freqüències relatives, i la modalitat epistémica és més freqüent en les conclusions. Igualment, s'han detectat diverses funcions estretament relacionades amb els significats modals. Així, la funció de mitigació i cortesia lingüística es relaciona en major mesura amb la modalitat epistémica i el mateix ocorre amb la formulació del significat inferencial, que es refereix a la necessitat epistémica. L'expressió de la factualidad i de la impossibilitat es nodreixen de la modalitat dinàmica, mentre que la predicció, I'organització dels continguts, l'autoritat i la recomanació es manifesten amb l'ús de la modalitat dóntica.

La tesi s'estructura en cinc capítols i les referències bibliogràfiques que s'inclouen al final d'aquest treball. 


\section{Summary}

The research article in the field of tourism studies has not been sufficiently studied, despite being a discipline strongly connected with a sector of economic growth for many countries. Other textual genres related to the tourism register such as the summary, brochures and web pages in the tourism register, the latter with great relevance for their clear social and economic impact of tourism studies, have enjoyed more scholarly attention. Scientific research in tourism, however, provides organisations, whether public or private, with relevant information for the improvement of the activity, which translates into recommendations that are often included in the conclusions of these studies. It is held, therefore, that there is a direct relationship between the development of the sector and empirical research, which, in turn, shows the interest in academic publications in leading journal. For this reason, this paper includes a formal characterisation of the genre into sections. This study is supplemented with a description of the recurrent lexicalgrammatical structures in each of the sections of the scientific article and the lexical and syntactic variables that characterise the text within the scientific register, both in terms of the complexity and the level of elaboration exhibited in the language used in these works. These structures are intended to signal the authors' perspective, among other aspects.

That said, the aim of this paper is to analyse modal verbs, their meanings and the existence of variation in the "introduction" and "conclusion" sections of the research article in the discipline of tourism. These texts have been taken from leading journals in the field and with a clear impact index in the discipline. Modal verbs reflect the speaker's point of view. As part of the methodology, corpus linguistics tools are used to allow the management of the texts and their consultation, as well as the excerption of concrete examples illustrating the forms, the semantic categories and the functions found in the texts studied. However, given that context is fundamental to the identification of modal meanings, part of the analysis of the texts requires direct visual recognition in each occurrence, as the same modal verb may entail several meanings. The analysis and discussion of the data is informed by studies in functional linguistics which allow us to count on the uses and functions of the different modal forms.

The conclusions indicate that there is indeed variation not only in terms of the forms used in each of the introductions and conclusions, but also in terms of meaning. In this sense, 
it is found that dynamic modality is more frequent in the introductions, according to their relative frequencies, and epistemic modality is more frequent in the conclusions. Likewise, several functions closely related to modal meanings have been detected. Thus, the function of mitigation and linguistic politeness is most closely related to epistemic modality, as is the formulation of inferential meaning, which refers to epistemic necessity. The expression of factuality and impossibility are nourished by the dynamic modality, while prediction, content organisation, authority and recommendation are manifested using deontic modality.

The thesis is structured in five chapters, and bibliographical references are included finally in this dissertation. 


\section{Agradecimientos}

Una tesis doctoral no es más que el momento para comenzar la trayectoria académica investigadora. Al mismo tiempo, es un momento especial en el que se reconocen las voluntades que apoyan para poder llevarla a término. Es ahí donde mis mayores agradecimientos surgen. En primer lugar, quisiera agradecer la excelente labor de dirección y de corrección de este trabajo a mis directores, el Dr. Francisco J. Álvarez Gil y la Dra. María Luisa Carrió Pastor. Ambos merecen mi más sincero agradecimiento por indicarme la dirección correcta al principio de mi investigación y por sus valiosas sugerencias para mejorar este trabajo en todas sus dimensiones. Es obvio que, sin su ayuda, este proyecto no se hubiese materializado.

En segundo lugar, agradezco a la Universidad Politécnica de Valencia, a su Escuela de Doctorado y al Departamento de Lingüística Aplicada por permitirme usar los recursos disponibles para poder culminar este trabajo. También me gustaría expresar mi agradecimiento al Dipartimento di Studi Linguistici e Culturali y a la Dra. Bondi por su ayuda durante mi breve estancia en la Università degli studi di Modena e Reggio Emilia. Así mismo, quisiera agradecer a mis compañeros del Departamento de Filología Moderna, Traducción e Interpretación por su colaboración para que pudiera asistir a congresos, realizar estancias y demás labores de investigación. 
En un plano más personal, agradezco la compañía y el apoyo de mis amigas y amigos en este trayecto; les agradezco especialmente su comprensión en aquellos momentos que no pude compartir con ellas por encontrarme inmersa en mi vida académica. A mi pareja, le agradezco todos los ratos en los que lo necesitaba y lo encontraba. A mis padres, razón por la que hoy me encuentro aquí, pues siempre han apoyado todas mis empresas. A ellos les debo mucho de quien soy hoy día.

Finalmente, dedico este trabajo a mi hijo Alejandro, quien tantas alegrías me ha dado. 


\section{A Alejandro, luz y motor de mi vida}





\section{Índice}

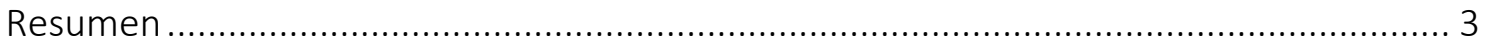

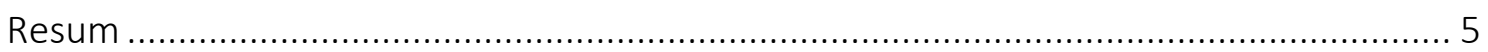

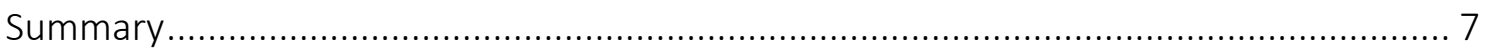

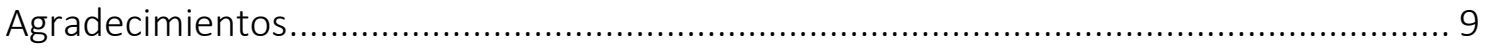

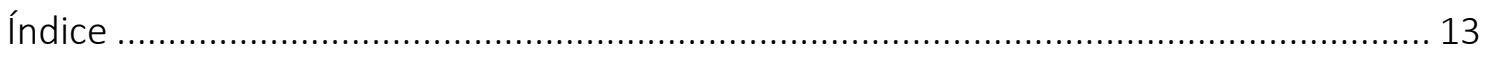

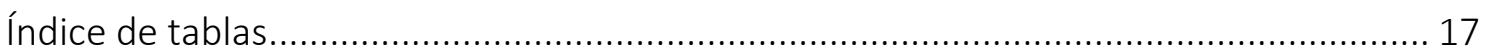

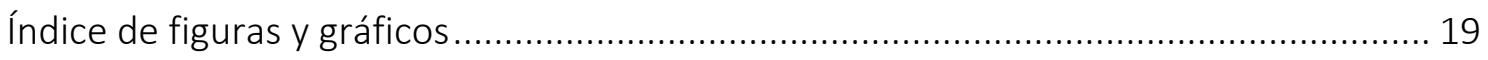

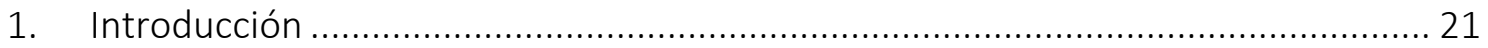

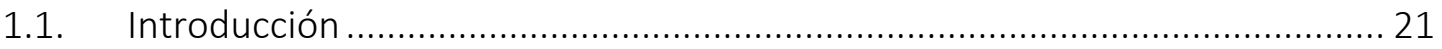

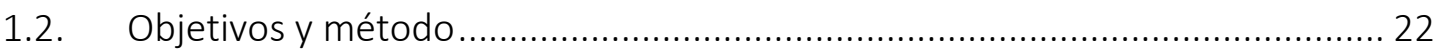

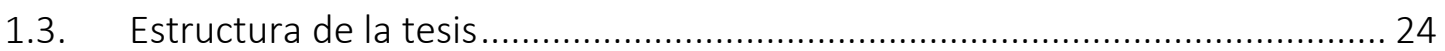

2. Marco teórico: Modalidad lingüística y el artículo de investigación en turismo como género textual 
2.1. Introducción

2.2. La modalidad lingüística. Definición, tipos y funciones.................................. 28

2.2.1. La tipología modal. Clasificación semántica ........................................... 33

2.2.1.1. Modalidad epistémica ................................................................ 35

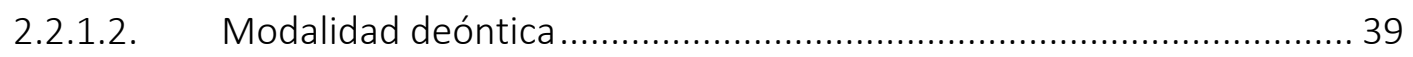

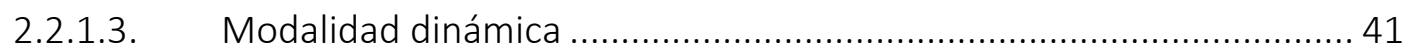

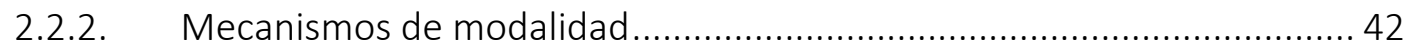

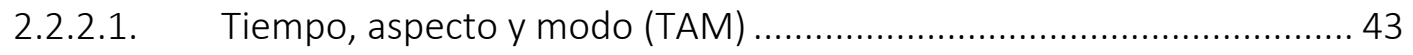

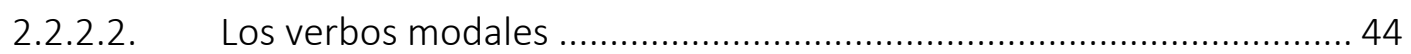

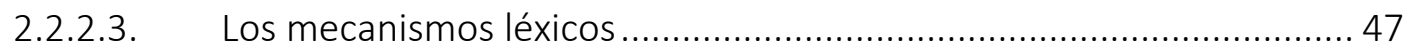

2.2.3. Objetividad, subjetividad e intersubjetividad .................................... 48

2.3. El artículo de investigación en turismo ....................................................... 50

2.3.1. Tipo de texto, género, registro en el contexto de la teoría sistémicofuncional (TSF). Descripción de conceptos .................................................... 51

2.3.2. El artículo de investigación en turismo. Estructura del artículo ............... 57

2.4. Lenguaje y secciones del artículo de investigación ......................................... 61

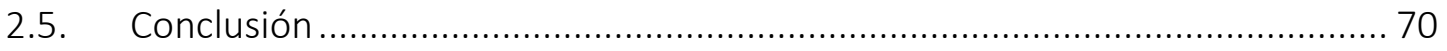

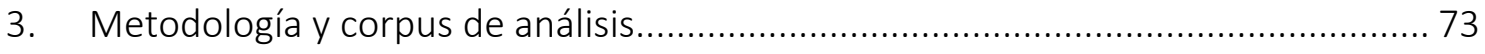

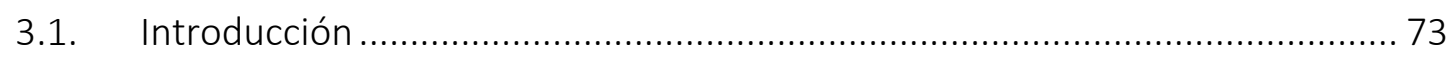

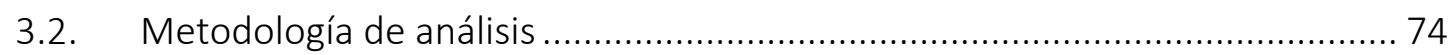


3.3. Metodología de compilación textual: Corpus de introducciones y conclusiones

de Al en turismo

3.4. Resumen 79

4. Resultados y discusión. La modalidad en introducciones y conclusiones en Al ....... 81

4.1. Introducción 81

4.2. Análisis del corpus: las formas de las perífrasis modales.....

4.3. Los significados de las perífrasis modales 104

4.3.1. Verbos perifrásticos epistémicos 106

4.3.2. Verbos perifrásticos con significado evidencial 109

4.3.3. Verbos perifrásticos dinámicos

4.3.4. Verbos perifrásticos deónticos

4.4. Las funciones de las perífrasis modales. 118

4.4.1. Mitigación, duda y cortesía lingüística

4.4.2. Función predictiva

4.4.3. Función de expresión de la factualidad 125

4.4.4. Inferencialidad

4.4.5. Indicar la organización

4.4.6. Expresión de la autoridad

4.4.7. Imposibilidad de realizar una acción

4.4.8. Formular recomendaciones para investigadores. 136

4.4.9. Formular recomendaciones para el sector turístico 
4.5. Conclusiones

5. Conclusiones.

5.1. Primera cuestión: Las formas de las perífrasis modales

5.2. Segunda cuestión: Los significados de las perífrasis modales

5.3. Tercera cuestión: Las funciones de las perífrasis modales

5.4. Investigación futura 155

Bibliografía 161

Corpus: referencias 183 


\section{Índice de tablas}

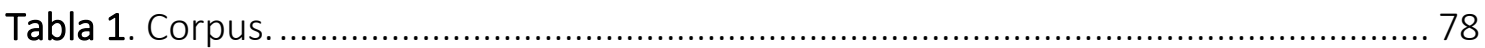

Tabla 2. FR de las perífrasis modales en introducciones y conclusiones. ....................... 82

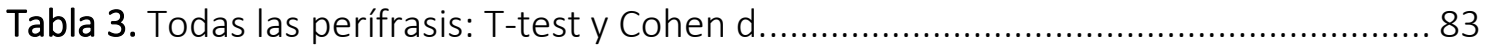

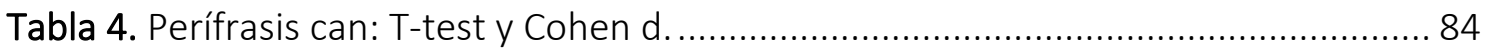

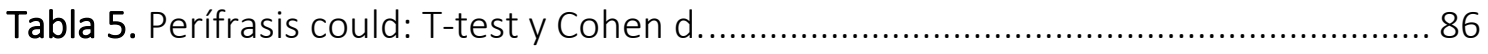

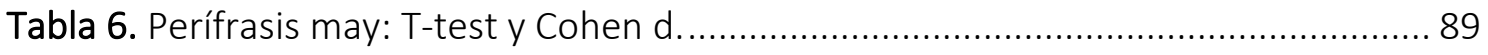

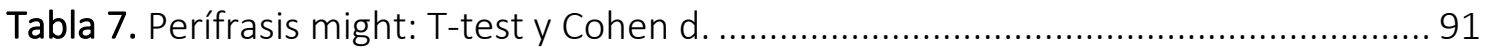

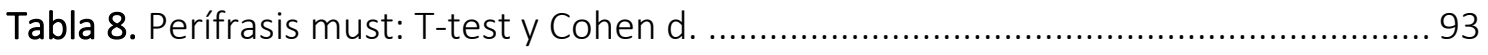

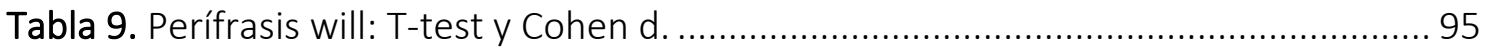

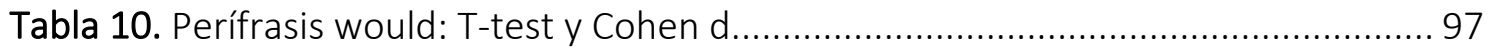

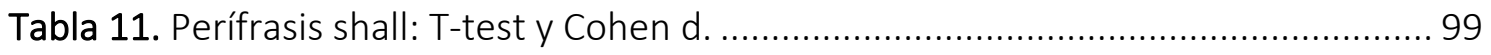

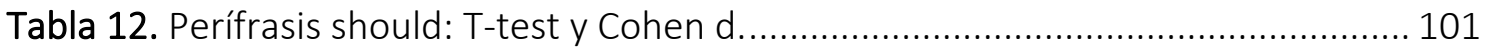

Tabla 13. Frecuencias relativas de aparición de significados modales por sección en turismo, ingeniería y lingüística. 105

Tabla 14. Frecuencias relativas de aparición de significados modales epistémicos por sección en los artículos de turismo. 106 
Tabla 15. Valores de log-likelihood y log ratio para medir el efecto de variación de presencia de perífrasis modales epistémicas. El asterisco indica mayor presencia en las conclusiones.

Tabla 16. Frecuencias relativas de aparición de perífrasis con significado evidencial por sección en los artículos de turismo

Tabla 17. Valores de log-likelihood y log ratio para medir el efecto de variación de presencia de perífrasis modales con significado evidencial. El asterisco indica mayor presencia en las conclusiones.

Tabla 18. Frecuencias relativas de aparición de significados modales dinámicos por sección en los artículos de turismo

Tabla 19. Valores de log-likelihood y log ratio para medir el efecto de variación de presencia de perífrasis modales epistémicas. El asterisco indica mayor presencia en las conclusiones.

Tabla 20. Frecuencias relativas de aparición de significados modales deónticos por sección en los artículos de turismo

Tabla 21. Valores de log-likelihood y log ratio para medir el efecto de variación de presencia de perífrasis modales deónticas. 


\section{Índice de figuras y gráficos}

Figura 1. Potencial genérico del artículo de investigación en turismo, según Alonso-

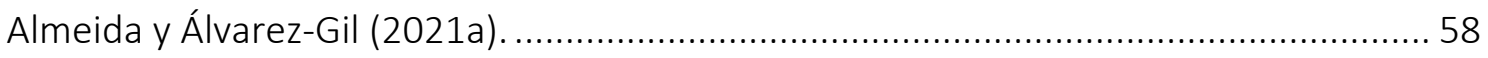

Gráfico 1. Secciones de potencial genérico y distribución, adaptado de Alonso-Almeida y

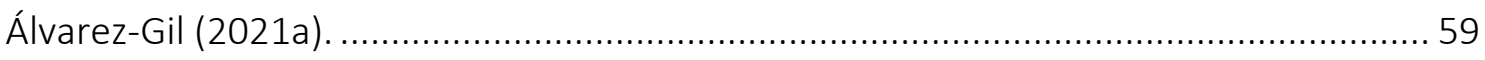

Gráfico 2. Distribución de perífrasis modales (FR 10,000 palabras). .............................. 82

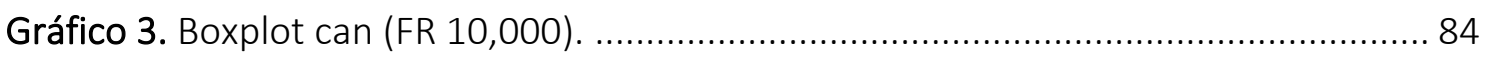

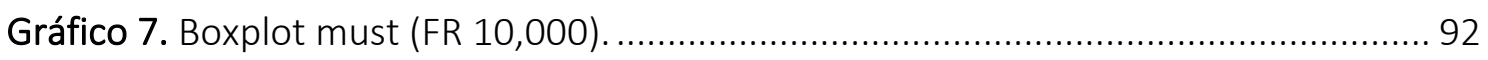

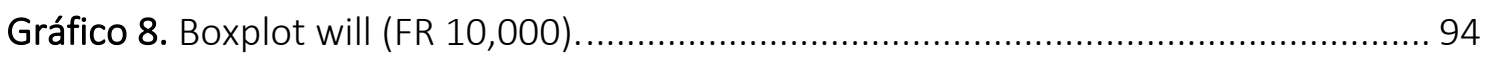

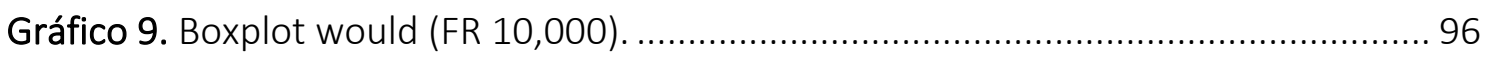

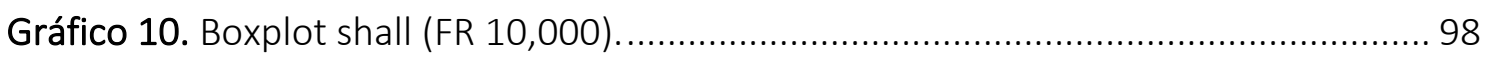

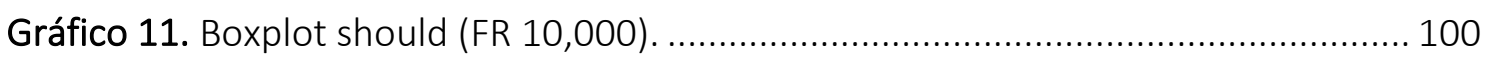

Gráfico 12. Frecuencias relativas de los significados modales por sección................... 104

Gráfico 13. Representación de la predicción a partir de la premisa partida. ................ 124 



\section{Introducción}

\subsection{Introducción}

El artículo de investigación en el campo de los estudios de turismo no ha sido un foco de atención para muchos investigadores (Lin \& Evans 2012), a pesar de ser una disciplina que representa un sector de desarrollo económico para numerosos países (Benkraiem et al. 2020: 25). Otro asunto es, sin embargo, el estudio de lengua que se ha llevado a cabo usando otros géneros textuales relacionados con el registro turístico, ya sea de manera central o tangencial como parte de un estudio interdisciplinar. En este sentido, se destacan los trabajos de Yui Ling Ip (2008), Chinanard (2008), Sulaiman (2014) y Carretero \& Zamorano-Mansilla (2015) en textos promocionales como el folleto; SuauJiménez (2007; 2012; 2016) en páginas web de turismo; Lin and Evans (2012), Dolnicar y Chapple (2015), Aluthman (2018) en textos de escritura académica; y Ahmed (2015), Sabila y Kurniawan (2020), Álvarez-Gil y Domínguez-Morales (2018) específicamente en el género abstract (resumen) de estos textos académicos.

Los trabajos mencionados dan una clara idea del impacto social y económico de los estudios turísticos. La realidad es, como se apunta en Alonso-Almeida y Álvarez-Gil (2021a), que la investigación en turismo provee a las organizaciones, ya sean públicas o privadas, de información relevante para la mejora de la actividad que se traducen en 
recomendaciones que, con frecuencia, se aportan en las conclusiones de estos trabajos. Se entiende, por lo tanto, que existe una relación directa entre el progreso del sector y la investigación empírica, lo que, a su vez, indica el interés por la publicación académica periódica. De aquí, se deduce que la forma macroestructural como la microestructural del artículo científico debieran ser analizadas y descritas con precisión con fines didácticos para presentes y futuros estudiosos en la disciplina.

Si bien se han realizado numerosos trabajos en aspectos concretos del lenguaje turístico como se acaba de mencionar, hasta la fecha solo existe un trabajo que cubre el género del artículo científico en turismo; este es Alonso-Almeida y Álvarez-Gil (2021a). En este trabajo, se incluye un análisis formal del género en una estructura de secciones, siguiendo la terminología en Martin (1984). El estudio se complementa con un análisis de las estructuras léxico-gramaticales recurrentes en cada una de las secciones del artículo científico y las variables léxicas y sintácticas que caracterizan al texto dentro del registro científico, tanto por la complejidad como por el nivel de elaboración que exhibe la lengua usada en estos trabajos. Además, los autores llaman la atención sobre el empleo de una serie de estructuras gramaticales que aparecen en las secciones con indicación de su variación significativa en cada una de ellas. Estas estructuras, como se señala en el trabajo, persiguen indicar la perspectiva de los autores, entre otras cuestiones. Así, se pueden encontrar en estas estructuras perífrasis modales, elementos intensificadores y los mitigadores, usos de voz pasiva, estructuras condicionales y las cláusulas con that.

\subsection{Objetivos y método}

Nuestra hipótesis de trabajo es que las perífrasis modales presentan funciones diferentes en las secciones denominadas "introducción" y “conclusión” del artículo de investigación 
en turismo motivadas por el interés comunicativo en cada una de ellas. Así, el objetivo principal de este trabajo es analizar las perífrasis modales, sus significados y la existencia de variación en las secciones mencionadas del artículo científico en un corpus de estas secciones en la disciplina de turismo; estos textos se han extraído de revistas en el campo y con un índice de impacto notorio en la especialidad. Las perífrasis modales reflejan el punto de vista del hablante, como se apunta en Palmer (2001) y en Biber et al. (1999), lo que la convierte en un mecanismo de comunicación interpersonal (Carrió-Pastor 2020c: 273). Dicho esto, este trabajo plantea las siguientes preguntas:

1) ¿qué formas de perífrasis modales son las que aparecen con mayor frecuencia en estas secciones del artículo académico?,

2) ¿qué significados modales son más frecuentes en la introducción y en la conclusión y si la variación existente es significativa?, y, finalmente,

3) ¿cuáles son las funciones que estas perífrasis modales cumplen en cada una de las secciones?

Para esto, se usan herramientas de lingüística de corpus que permiten la gestión de los textos y su consulta, además de la extracción de ejemplos concretos que ilustren las formas, las categorías semánticas y las funciones que se encuentren en los textos estudiados. Sin embargo, dado que el contexto es fundamental para poder identificar los significados modales, parte del análisis de los textos requiere de un reconocimiento visual directo de cada caso. El papel del contexto a la hora de especificar el significado que tiene un determinado verbo modal ha sido descrito con anterioridad (Alonso-Almeida 2015a). Una misma perífrasis modal puede indicar diferentes significados, por lo que, sin ayuda del contexto, no sería razonable esperar una categorización precisa de estas formas verbales. En otras palabras, la categorización de los verbos modales mediante un proceso 
de etiquetado automático no daría resultados, ya que el software actual en lingüística de corpus no puede analizar la semántica y la pragmática de las formas que se analizan con precisión y fiabilidad. La flexibilidad semántica de las perífrasis modales las hace únicas, pero también esto significa que debemos prestar especial atención en seleccionar los contextos adecuados para no errar en la identificación de los significados que estas formas representan. De igual manera, tampoco se puede automatizar la categorización de las funciones pragmáticas que estas formas modales tienen en cada caso en los textos.

Esto no significa que el uso de las herramientas informática no sea útil en este trabajo. Por un lado, la búsqueda manual de las perífrasis modales requeriría mucho tiempo y no estaría exenta de errores, ya que algunas formas se podrían quedar atrás. Por otro lado, las búsquedas automáticas permiten recuperar todos los casos para una consulta concreta, que puede incluir a la perífrasis modal con una o más formas concurrentes. Además, el software de búsqueda ofrece cada una de las formas encontradas en su cotexto, lo que mejora el análisis. Estas búsquedas informatizadas facilitan el cálculo de estadísticas sobre la frecuencia de formas por sección para detectar la existencia de variación. Todo ello justifica el uso de una metodología combinada para recuperar y categorizar con seguridad los verbos modales.

\subsection{Estructura de la tesis}

Esta tesis doctoral se estructura en cinco capítulos, además de las referencias que se incluyen al final del trabajo. En este capítulo, se presenta la justificación de la investigación para realizar el estudio de los verbos modales en los artículos de investigación dentro de una perspectiva del género textual que especifica las funciones de las secciones de estos textos. El capítulo segundo describe el concepto de modalidad. 
Se propone aquí la definición de este término que se usa en el análisis de las perífrasis que se encuentran en el corpus de trabajo. Además, se explica la tipología modal en la que se clasifican dichas perífrasis, así como los mecanismos modales para encuadrar el tipo de los que se analizan en esta tesis. Relacionado con el concepto de modalidad, se incluye una descripción de los conceptos de objetividad, subjetividad e intersubjetividad, que determinan la perspectiva del hablante y la manera en la que se relaciona con el texto y con la veracidad de lo que expresa. Estos términos indican, entre otros aspectos, si la información aportada en un texto representa un punto de vista único y compartido y permite explicar la razón por la que se ha usado un mecanismo modal frente a otros.

También en este capítulo, se describe el artículo de investigación en turismo y se caracteriza como género textual desde una perspectiva sistémico-funcional. Para esto, se definen conceptos relacionados como el tipo de texto, el género textual y el registro. De la aplicación de estos, se deduce una estructura del artículo científico en turismo que se sustenta, además, en un uso concreto de la lengua con una serie de expresiones recurrentes y típicas en cada una de las secciones. Muchas de estas expresiones parecen tener una relación directa con la función que cumple cada sección.

El capítulo tres se dedica a las cuestiones metodológicas y de compilación del corpus de trabajo. Se dedica aquí un apartado inicial a las cuestiones metodológicas de construcción y uso del corpus de trabajo y se explican los métodos estadísticos que se emplean en el análisis de los datos en cada una de las secciones del capítulo de resultados y discusión.

En el capítulo cuatro, se analizan las perífrasis modales en las introducciones y en las conclusiones del artículo de investigación en turismo. Se presenta una clasificación y una discusión de los resultados. Aquí, no solo se describen las formas y los significados 
modales en los textos estudiados, además se incluyen las funciones modales que cumplen los mecanismos modales analizados.

Finalmente, se ofrecen las conclusiones. Aquí, se establecen las principales aportaciones de este trabajo y su relevancia para el género del artículo científico, indicando la variación existente entre las introducciones y las conclusiones y las motivaciones para dicha variación. Se complementa este aspecto con un apartado que se dedica a la presentación de ideas para la investigación futura con textos de artículos de investigación en turismo. Al término de este capítulo se proporcionan las referencias citadas en esta tesis y un apéndice con las referencias bibliográficas del corpus analizado. 


\section{Marco teórico: Modalidad lingüística y el artículo de investigación en turismo como género textual}

\subsection{Introducción}

En este capítulo, se pretende mostrar una definición de modalidad que se seguirá en el análisis e interpretación de los verbos perifrásticos obtenidos mediante consulta informatizada de un corpus de introducciones y conclusiones de artículos científicos en el registro turístico. Aquí, se incluirá una clasificación semántica de la modalidad, siguiendo, principalmente a Palmer (2001), van der Auwera y Plungian (1998) y Nuyts (2001), entre otros. El estudio de los verbos modales, y de la modalidad en general, en textos de especialidad no es realmente novedosa, como se demuestra en la literatura existente (cf. Giltrow 2005; Efstathiadi 2010; Cheng \& Cheng 2014; Alonso-Almeida 2014; Ge 2015; Carrió-Pastor 2017; Álvarez-Gil 2018; Carrió-Pastor 2020a). La novedad de este estudio radica principalmente en la selección de dos secciones fundamentales del artículo de investigación para la recepción y el impacto de este como aportación científica, como son la introducción y la conclusión, y mi interés por los textos turísticos.

Como se ha expresado ya en Álvarez-Gil y Domínguez-Morales (2018), el artículo de investigación en turismo es con mucho uno de los menos explorados. Puesto que los textos que se compilan pertenecen precisamente a este tipo de artículos, se presenta 
también un análisis de este género en la especialidad de turismo, siguiendo la descripción realizada por Alonso-Almeida y Álvarez-Gil (en prensa, 2021a). Esta caracterización es útil para identificar las secciones "introducción" y "conclusión" en estos artículos. Con este fin, se usará como marco de referencia la teoría sistémico-funcional, según Halliday y Matthiessen (2013; 2014). Junto con el estudio del género textual, se explicarán aspectos relacionados con el registro, además de los tipos de texto que configuran los géneros textuales.

En la sección 2.2, se incluye una definición de la modalidad, que surge de estudios previos en la materia. Se incluye una taxonomía modal, según criterios semánticos, que se usará en la clasificación de los datos. Se describen cada una de las categorías incluidas en el orden tripartito seleccionado con ejemplos extraídos de fuentes reconocidas. En la siguiente sección, se ofrece una definición del artículo de investigación como género después de esbozar cuestiones relativas a todos los conceptos mencionados anteriormente de tipo de texto, género y registro en la teoría funcional, lo que permite la caracterización de los textos que se analizan en esta tesis doctoral. La última sección incluye un resumen de lo expuesto en este capítulo.

\subsection{La modalidad lingüística. Definición, tipos y funciones}

El concepto de modalidad se entiende de manera desigual en la literatura científica al respecto. Algunas definiciones de modalidad son las siguientes:

Palmer (2001: 1): Modality is concerned with the status of the proposition that describes the event.

Gotti y Dossena (2001: 10): In particular, modality enables the locutor to make important strategic choices over very subtle gradable scales for the conveyance of attitudinal aspects associated to one's degree of commitment to such issues as 
possibility, obligatoriness, ability and so on. Indeed, the concepts of 'necessity', 'possibility', 'probability' and 'impossibility' are at the core not only of linguistic studies but also of modal logic, and have been a topic of central interest and intense study since classical times...

From a linguistic point of view, modality is a complex concept to categorise and qualify in its different forms. There are various ways in which a speaker may add an overlay of meaning to the neutral semantic value of the proposition. One of these is mood; indeed, as remarked by Palmer (1986: 21), "the distinction between mood and modality is [...] similar to that between tense and time", in so far as mood is a morphosyntactic category of verb forms, expressing the degree or kind of reality assigned to a sentence. Several moods are to be distinguished, the three basic types being indicative, imperative and subjunctive.

Narrog (2005: 184): Modality is a linguistic category referring to the factual status of a state of affairs. The expression of a state of affairs is modalized if it is marked for being undetermined with respect to its factual status, i.e. is neither positively nor negatively factual.

Portner (2009: 2): Modality is the linguistic phenomenon whereby grammar allows one to say things about, or on the basis of, situations which need not be real.

Saeed (2016: 134): Modality is a cover term for devices which allow speakers to express varying degrees of commitment to, or belief in, a proposition.

Rocci (2017: 3): Modality is the semantic category associated with the basic human cognitive ability of thinking that things might be otherwise, that is thinking of alternatives: situations other than what is the case. Modality refers generally to the linguistic means that allow "one to say things about, or on the basis of, situations which need not be real" (Portner 2009: 1, emphasis is ours).

Alonso-Almeida y Álvarez-Gil (2020: 62-63): Modality is the term used in linguistics to refer to the expression of a speaker's evaluation of an event in terms of such notions as probability, possibility, obligation, permission and necessity, among other more finegrained attitudes towards the propositional content framed by the modal particle. 
Estas definiciones muestran la visión desigual que existe entre los lingüistas que se acercan a este concepto. Se trata, pues, de un fenómeno que puede representar diferentes realidades, pero todos los acercamientos coinciden en que modalidad sirve para matizar el significado de un evento lingüístico. Uno de los aspectos más interesantes es qué es lo que se modaliza. Por una parte, Palmer (2001) y Saeed (2016) hablan de proposiciones mientras que Portner (2009) y Rocci (2017) se refieren a situaciones, Alonso-Almeida y Álvarez-Gil (2020) los denominan eventos, y, finalmente, Narrog (2005, 2012) prefiere usar el término state of affair. Es relevante, entonces, señalar aquí el significado técnico de conceptos proposición y state of affairs, pues el término situación es más evidente. Así, se definen estos dos elementos en el ámbito de la lingüística de la siguiente manera:

\section{PROPOSICIÓN}

The word 'proposition' is used in pragmatics in a technical sense which comes from logic and philosophy. The term can be defined by the claim that every statement that is either true or false expresses a proposition. For example the statement that 'All men are equal' expresses the proposition that all men are equal.

The same proposition can be expressed in different languages: 'Elephants are scared of mice' and 'Les éléphants ont peur des souris' express the same proposition (Allott 2010: 156).

\section{STATE OF AFFAIRS}

Phenomena, events, situations, states of affairs are commonly supposed to be genuinely-in-the-world, and even Strawson admits events are so. Yet surely of all of these we can say that they are facts (Vendler 1967: 122) [...] It seems to me, therefore, that states of affairs due to human action can be viewed in two ways. Inasmuch as they are considered in connection with the actual or possible intention of the agent we are inclined to speak of them as results. If, however, they are considered in abstraction from such intention, we prefer to call them consequences (Vendler 1967: 161). 
De manera simple, diremos que la proposición hace referencia a la expresión verdadera o falsa de significado en el campo de la lógica-semántica, mientras que el término state of affair alude a un hecho, que puede ser resultado o consecuencia de la acción humana. En este trabajo, dado que se estudia la modalidad en los verbos modales, parece apropiado usar el término proposición, pues responden a una misma naturaleza filosófica de la lengua.

Tras esta aclaración, y volviendo a las definiciones de modalidad expuestas arriba, se destaca en todas ellas su uso evaluador y matizador de la proposición a la que la partícula modal acompaña para precisarla. Así, la percepción del hablante conforme a dicha proposición se encapsula en dicha forma modal para expresar, por ejemplo, obligación o probabilidad, según sea el caso. Este aspecto evaluativo de la modalidad implica que su análisis pueda ser encuadrado dentro de lo que se denominan estudios de perspectiva o, en inglés, stance, como se apunta en Alonso-Almeida (2015b: 2):

Stance indeed refers to different phenomena in language, and so it is generally the umbrella term for notions, such as epistemic stance ([Biber \&] Finegan 1989), commitment (Caffi 1999; Caffi 2007; Del Lungo Camiciotti 2008), mitigation (MartínMartín 2008; Alonso-Almeida 2015a), reinforcement or strengthening (Brown 2011), intensification (González 2015), authority, involvement and hedging (Hyland 2005a; Hyland 1998), assessment (Goodwin 2006), modality and evidentiality (Chafe 1986; Chu et al. 2011; Fairclough 2004; Marín Arrese 2009; Carrió-Pastor 2012; Pic \& Furmaniak 2012; Goodwin 2006), affect (Martin 2000; Martin \& White 2005), and vagueness in language (Cutting 2007).

En algunos casos, como Saeed (2016), la definición de modalidad incluye la noción de compromiso del hablante con respecto a la veracidad de la proposición expresada, lo que puede incluir cierta gradación, como se apunta en esta fuente. También se precisa en la 
definición de Gotti y Dossena (2001) la diferencia entre modalidad y modo, siendo este último de tipo morfosintáctico y que refleja aspectos de la realidad referida en la proposición. Un ejemplo sería el uso del imperativo para expresar orden frente al uso del subjuntivo para indicar hipótesis con respecto a la posible realización de la acción que se describe. Además del modo, otros elementos lingüísticos comunican modalidad como los verbos o perífrasis modales y los clíticos, como señala Palmer (1986: 33 ff). En definitiva, lo que este autor viene a afirmar es que la modalidad puede manifestarte tanto morfológicamente como con mecanismos léxicos. El caso de los modales, como así lo afirma Aikhenvald (2004), se considera a media distancia entre la gramática y el léxico.

Como se ha visto son varios los aspectos que incluyen las definiciones recogidas al comienzo de esta sección, lo que refleja, además de la multiplicidad de enfoques, la diversidad terminológica con respecto a estos aspectos, por ejemplo: evento y hecho. Es lógico, pues, que plantee una simplificación que permita el análisis de los datos que ofrezca nuestro corpus. Así, se define este concepto para el objetivo de esta tesis de la manera siguiente:

Modality is the term used in linguistics to refer to the expression of a speaker's evaluation of an event in terms of such notions as probability, possibility, obligation, permission and necessity, among other more finegrained attitudes towards the propositional content framed by the modal particle (Alonso-Almeida y Álvarez-Gil 2020: 63).

Se entiende, en este contexto, el término evaluación en el sentido expresado por Hunston y Thompson (2000: 5):

evaluation is the broad cover term for the expression of the speaker or writer's attitude or stance towards, viewpoint on, or feelings about the entities or propositions that he 
or she is talking about. That attitude may relate to certainty or obligation or desirability or any of a number of other sets of values. When appropriate, we refer specifically to modality as a sub-category of evaluation.

La perspectiva del significado es un criterio de clasificación de la modalidad, como se explicará en la siguiente sección.

\subsubsection{La tipología modal. Clasificación semántica}

La manera en la que la modalidad puede categorizarse varía según las escuelas de pensamiento y los críticos que realizan estas clasificaciones. Sin embargo, la clasificación semántica de Lyons (1977) en modalidad epistémica y modalidad deóntica parece ser de las más aceptadas y que Palmer (1986: 19) mantiene. Esta misma clasificación bipartita se encuentra en Biber et al. (1999: 485), pero las categorías se denominan modalidad intrínseca y modalidad extrínseca, que definen así:

Each modal can have two different types of meaning, which can be labeled intrinsic and extrinsic (also referred to as 'deontic' and 'epistemic' meanings). Intrinsic modality refers to actions and events that humans (or other agents) directly control: meanings relating to permission, obligation, and volition (or intention). Extrinsic modality refers to the logical status of events or states, usually relating to assessments of likelihood: possibility, necessity, or prediction.

Palmer (2001) hace otra clasificación que distingue entre la modalidad proposicional y la modalidad de evento. La primera de ellas incluye la modalidad epistémica (que se explicará en la sección 2.3.1), y la evidencialidad, que se refiere a la fuente o el modo de información. Este último fenómeno ha sido estudiado con frecuencia en estas últimas décadas (cf. Chafe 1986; Willett 1988; Leavitt, Chafe \& Nichols 1991; Lazard 2001; 
Plungian 2001; Viechnicki 2002; Boye \& Harder 2009; Mushin 2013; Haßler 2011; AlonsoAlmeida 2015a; Greco 2018) y que se analiza como un mecanismo indicador del punto de vista del hablante con respecto a la información que se ofrece. Este tipo de modalidad no será exactamente objeto de estudio en este trabajo, si bien se hará mención más adelante de un tipo específico de estos mecanismos denominado evidencialidad inferencial que van der Auwera y Plungian (1998) identifica como necesidad deóntica.

La modalidad de evento expresa una actitud con respecto a la información aportada y se divide en modalidad deóntica y modalidad dinámica que se relacionan con significados de obligación y habilidad, respectivamente, entre otros.

En este estudio, se usa una categorización tripartita donde se incluyen estos tipos junto con la modalidad epistémica, es decir: modalidad epistémica, modalidad deóntica y modalidad dinámica. Sin embargo, antes de seguir, parece conveniente hacerme eco de otros tipos de modalidad mencionada con frecuencia en la literatura. Estos son la modalidad existencial y la modalidad alética, según von Wright (1951). La modalidad existencial, que se refiere a los modos de existencia, no se debe considerar, como apunta Palmer (1990: 6), ya que este aspecto se encuentra en las otras modalidades. Un ejemplo de este tipo de modalidad recogido en Palmer (1990: 110) es el siguiente, donde might existencial presenta un uso cercano a would, como se destaca en la misma fuente:

In those days we might go for a walk through the woods.

Igualmente, Palmer (1990: 6) reconoce que la modalidad alética representa un "main concern of logicians, but it has little place in ordinary language". En su ejemplo,

John is a bachelor, so he must be unmarried. 
donde must tiene un valor alético, pues, siguiendo a Alonso-Cortés (2008: 256), este tipo de modalidad se mueve en parámetros de probabilidad y necesidad. En la misma línea de la modalidad existencial, es más útil en lógica-filosófica, pero se entiende que no tiene la misma repercusión en el lenguaje natural, como dice Palmer (1990).

\subsubsection{Modalidad epistémica}

La modalidad epistémica se relaciona con "matters of knowledge or belief on which basis speakers express their judgements about state of affairs, events or actions" (Hoye 2008), y, además, "[...] applies to assertions and indicates the extent to which the speaker is committed to the truth of the proposition" (Bybee, Perkins \& Pagliuca 1994: 179). Esto deja en claro que este tipo de modalidad se asocia frecuentemente con la idea de verdad con respecto a la información proposicional que se modula, como se aprecia en los siguientes $\operatorname{casos}^{1}$ :

Changing the climate and weather conditions of any destination may affect the tourists' comfort and travel decisions, and due to the changing demand pattern and tourist flows, tourism businesses and host communities suffer. Cancellation of any trip due to bad weather causes dissatisfaction and those affected may avoid visiting the destination again (Mahnas 2016).

Sharing conservation revenue with communities surrounding parks could demonstrate the link between ecotourism and local communities' economic development, promote a positive view of land restitution involving parks, help address skewed distribution of income in the vicinity of parks and act as an incentive for local communities to participate in conservation even more (Dikgang y Muchapondwa 2017).

\footnotetext{
${ }^{1}$ En la descripción de los tipos de modalidad de esta sección, los ejemplos se han tomado de Álvarez-Gil y Domínguez-Morales (2018).
} 
En el primer ejemplo, el verbo may tiene una clara función de mitigación del contenido proposicional, ya que los autores buscan proporcionar información que se estima que sucederá en el futuro. En este texto, los autores indican que existe la probabilidad de que aspectos como el cambio de las condiciones climáticas tengan una consecuencia en la toma de decisiones para realizar un viaje vacacional. También podría ser el caso de que "may" se use aquí como una estrategia de cortesía negativa para evitar la imposición a sus lectores (Alonso-Almeida 2014; Alonso-Almeida 2015a; Carrió-Pastor 2016; CarrióPastor 2020b). En el caso del verbo could en el segundo ejemplo, indica que existe una probabilidad indeterminada de que compartir los ingresos de conservación con las comunidades locales produzca beneficios para la industria y para cada comunidad específica.

Un caso especial de valor epistémico es el llamado necesidad epistémica por van der Auwera y Plungian (1998) que representa un espacio modal intermedio entre lo que es posible y necesario según una realidad determinada, como en el siguiente ejemplo de autoría propia:

She must have been crying. Her eyes are red and watery.

Aquí, el verbo must indica un razonamiento inferencial por el que el autor presenta el contenido proposicional como una conclusión de acuerdo a los contextos seleccionados en el proceso interpretativo; esto se considera también inferencialidad evidencial (evidentiary inferentiality), que Alonso Almeida (2015c), en su interpretación sobre el uso de seem y parecer en textos médicos modernos anteriores a 1700, describe como un mecanismo indicador del papel del hablante con respecto a la elaboración de la información. En palabras del autor, este mecanismo indica que la "information has been 
construed upon the consideration of evidentiary variables that lead the speaker to make a particular claim" (Alonso-Almeida 2015c: 130).

Como se ha sugerido anteriormente, las perífrasis modales pueden denotar la voluntad de los autores por mitigar el contenido proposicional del acto de habla, lo que está relacionado con la expresión de la duda, o la ausencia de certeza absoluta, y la cortesía lingüística. Como se apunta en Alonso-Almeida (2015a: 37), "mitigation is indeed a complex linguistic concept", pues depende, en parte, del contexto en el que se desarrolle, así como la intención del hablante en señalar el deseo de atenuar la dureza de su aseveración. En principio, la mitigación se lleva a cabo, sobre todo, mediante estructuras epistémicas como las que se han visto aquí, si bien Alonso-Almeida (2021) afirma que esta capacidad de atenuar el contenido de la proposición no es exclusiva de este tipo de modalida e incluye las formas dinámicas también, como se verá en la sección 2.2.1.3.

En la literatura científica, las estructuras epistémicas con valor atenuante también se les conoce en inglés por el término de hedging, por lo que las perífrasis epistémicas encajarían como mecanismos capaces de reducir la fuerza elocutiva de la proposición (Hyland 1998; Vassileva 2001; Hu \& Cao 2011; Carrió-Pastor 2014; Alonso-Almeida \& Carrió-Pastor 2016; Moskowich \& Crespo 2019; Flores 2020; Carrió-Pastor 2020b).

En gran medida, esta función de atenuar el contenido proposicional sigue una tradición de escritura académica que pretende evitar tanto la imposición del punto de vista, como la confrontación por razones de discrepancias. En este sentido, se relaciona con el concepto de cortesía lingüística que se explica en Brown y Levinson (1987), aunque, como dice Alonso-Almeida (2015a: 38), con respecto a esta relación entre lo epistémico y la 
cortesía, la relación no puede entenderse como un principio básico de correspondencia en todos lo casos:

Indeed the hedging function of mitigation is one of the commonest pragmatic meanings of modality in context, although it is by no means the only one (Hu and Cao 2011; Hyland 1998; Vassileva 2001). For this reason and contrary to the parallelism between mitigation and politeness put forward in Brown and Levinson (1987: 42), politeness should not be readily inferred from the use of epistemic modals, and certainly not from the use of evidentials in discourse.

La cortesía se basa en el concepto de face que se define como "the public self-image that every member wants to claim for himself" (Brown y Levinson 1987: 61). Se trataría de proteger esta imagen pública, lo que daría lugar a estrategias de cortesía positiva y estrategias de cortesía negativa. En el caso de las primeras, su uso está destinado evitar en la medida de lo posible usar lenguaje que comprometa la buena imagen de la persona que escucha o lee. En el caso de las segundas, estas estrategias evitan la imposición del punto de vista para que no se produzca una reacción por parte de la persona que escuche o lea, lo que pondría en riesgo su buena imagen. En este sentido, la mitigación presentaría la información despojada de certeza absoluta, por lo que se entenderían diferentes grados de seguridad con respecto al contenido proposicional, llegando incluso a referir duda, si bien la persona que lee o habla esté segura de lo que dice o escribe.

Estas cuestiones planteadas sobre la cortesía y la ausencia de imposición forman parte indispensable del comentario de las perífrasis modales encontradas en el corpus de trabajo en el capítulo 4. 


\subsubsection{Modalidad deóntica}

La modalidad deóntica se relaciona con la "[...] necessity of acts in terms of which the speaker gives permission or lays an obligation for the performance of actions at some time in the future" (Hoye 1997: 43). Según apunta Collins (2009: 22), la modalidad deóntica

occurs when the factors impinging on the actualisation of the situation referred to in the utterance involve some type of authority - as when a person or a set of rules or a social convention is responsible for the imposition of an obligation or a granting of permission.

La modalidad deóntica se caracteriza por la obligación o la necesidad de llevar a cabo una acción de acuerdo con una fuerza deóntica que puede ser interna o externa al hablante, como se ejemplifica en los siguientes fragmentos con should, will y must:

The results of the research help to provide a better understanding of the conditions needed to develop and manage sustainable tourism in post-conflict environments. In so doing, it should be possible to make better policy decisions, with particular reference to social and urban interventions on planning, design and entrepreneurship (Farsari 2018).

There is a need for detailed and comprehensive legislation protecting the rights and needs of disabled tourists, particularly in view of Dubai's successful bid to host Expo 2020. This need will, no doubt, be fulfilled in light of the recent announcement that Dubai will enact its own disability law intended to make the emirate fully accessible to persons with disabilities (Morris y Kazi 2014).

For golfers to 'enact' golf, in its current form, tied to designated playing fields (CeronAnaya, 2010), a material landscape must first be 'appropriated for one use and thus unavailable for others' (Klein, 1999: 214) (Kang et al. 2018). 
El uso de should en el primer ejemplo refleja la opinión de los autores sobre cómo se estima que las cosas son necesariamente como se expresa de acuerdo con la evidencia presentada en la investigación que se ha llevado a cabo. Los fuertes efectos que este modal puede traer en términos de obligación se mitigan de alguna manera por el uso de be possible después del verbo modal. Igualmente, en el caso de will en el segundo ejemplo, indica la posición firme de los hablantes con respecto al contenido proposicional, lo que, además, refuerzan con el adverbial "no doubt". En el último ejemplo, la perífrasis must muestra claramente el sentido de obligación acerca de la realización de la acción descrita en la proposición, que incluye el adverbial first incidiendo en la necesidad de que dicha acción se haga y que sea en el orden indicado.

En este sentido, la modalidad deóntica se presenta como idónea para la expresión de autoridad en el artículo académico, como explica Álvarez-Gil (2021: 261):

The functions of modal verbs driven by these notions of necessity and possibility contribute patently to the elaboration and presentation of knowledge. This includes the idea of academic authority, which stands vital in technical accounts of natural phenomena. Authority, however, is more positively on a par with expertise than with imposition... Deontic modals are contextually justified to indicate perceptions of mild obligation, desirability, volition, advisability and recommendation in the form of prescribed directions to achieve a particular goal. Precisely, the use of directives modulated by means of modal verbs, indicating necessity slightly attenuates a directive that could have been construed with an imperative form.

En la discusión de los resultados del análisis de las perífrasis modales en el corpus de introducciones y conclusiones de los artículos científicos, se notará que una de las funciones de las formas deónticas es mostrar la convicción de los autores en la 
formulación del contenido mediante nociones de necesidad, deseo o consejo, entre otras.

\subsubsection{Modalidad dinámica}

La modalidad dinámica es un término más complejo, ya que con frecuencia se confunde con la modalidad epistémica, pues ambos tipos de modalidad se refieren a lo que es posible, si bien la posibilidad referida es de diferente naturaleza. Alonso-Almeida y CarrióPastor (2017: 279) definen la modalidad dinámica de la siguiente manera:

In dynamic modality, conditions are internal, and it involves senses of willingness and ability on the part of the speaker or writer. Dynamic modality refers to the potentiality of the speaker to develop an action, whether this potentiality be internally or externally motivated, as the enabling circumstances are external or internal.

Según Alonso-Almeida (2015d), la modalidad dinámica aparece con frecuencia en los textos de carácter técnico-científico e indica que este tipo de modalidad "is used even when the context implies assurance" (Alonso-Almeida 2015d: 404). Advierte que esta seguridad y confianza en la realización del evento descrito en la proposición se consigue teniendo en cuenta las capacidades de un objeto o de una persona en particular, por lo que más que un efecto modulador, indicaría factualidad. Esto se percibe en el ejemplo siguiente donde el uso de will ilustra cómo los autores son conscientes de lo que su trabajo puede aportar a la industria alimenticia y que identifican como un hecho y que, por lo tanto, marcan mediante will.

The article will be of value to practitioners, researchers, policy makers and other stakeholders involved in the food industry (Taylor et al. 2015). 
Se tiende a confundir la modalidad dinámica y la modalidad epistémica por la dificultad que representa qué tipo de posibilidad se refiere en cada ejemplo. Para esto es fundamental el contexto, pues se podrá determinar de esta manera si se trata de un hecho posible debido a una cualidad o a una hipótesis. Además, uno de los elementos que llevan a la confusión es la posibilidad de expresar cortesía, evitando la imposición del punto de vista, a través de la modalidad dinámica, lo que normalmente es realizado por medio de los elementos epistémicos. Esta idea se recoge en el estudio que hace AlonsoAlmeida (2021) sobre las perífrasis modales dinámicas:

Entre las formas más usadas para significar cortesía están may y could, probablemente como extensión de sus equivalentes epistémicos... La función pragmática de los modales dinámicos es principalmente manifestar cortesía mediante la atenuación del contenido proposicional para evitar imponer el punto de vista de los hablantes. La modalidad dinámica, que se refiere a una posibilidad basada en la disposición y el potencial para realizar una acción, se basa en esta característica para conseguir este efecto mitigante, pues se presenta a menudo la información aportada como una perspectiva y no como un hecho constatado. En esta línea, se entiende, y es la idea que he tratado de defender en este trabajo, que la modalidad dinámica en el corpus de trabajo persigue con frecuencia mostrar la identificación del papel del hablante en la elaboración de la información, ya sea por referencia a características internas o externas de los participantes (Alonso-Almeida 2021: 545).

\subsubsection{Mecanismos de modalidad}

Son varios los mecanismos que se relacionan con la expresión de la modalidad y estos incluyen elementos morfológicos y léxicos. Entre los primeros, se encuentran la indicación de tiempo, aspecto y modo. Los verbos modales, igualmente denominados perífrasis modales, se encuentran en la intersección entre el significado gramatical y significado léxico, como indica Aikhenvald (2004), y tienen la capacidad de indicar la 
perspectiva del hablante. Los elementos puramente léxicos son, por ejemplo, sustantivos y adjetivos, si bien no son objeto de análisis en este trabajo. Se describen todos estos mecanismos en esta sección.

\subsubsection{Tiempo, aspecto y modo (TAM)}

Comrie define el tiempo como la "grammaticalised expression of location in time" (1985: 9), lo que significa que una lengua puede codificar mediante rasgos morfológicos el momento en el que se realiza una acción determinada. En los ejemplos Me quedo en casa para comer con Inés y Me quedé en casa para comer con Inés, el tiempo en el que se produce cada evento viene expresado por el uso de las desinencias $-o$ y -é, para la primera persona del singular del presente y del pasado, respectivamente. En otras palabras, el tiempo verbal posee un valor deíctico.

La relación del tiempo con el aspecto, como apuntan Hogeweg, de Hoop y Malchukov (2009: 1), no presenta gran complejidad, si bien la proximidad entre estos dos conceptos es evidente. Si bien el tiempo indica cuándo una determinada acción se lleva a cabo, el aspecto puede matizar la manera en la que se realiza una acción, incluso si es el mismo tiempo verbal, como se aprecia en estos ejemplos: Escribo una monografía sobre los verbos modales en lengua inglesa y Estoy escribiendo una monografía sobre los verbos modales en lengua inglesa. Está claro que, mientras que los dos eventos se desarrollan en el presente, existe cierta perspectivización en el uso del presente escribo y del progresivo estoy escribiendo, que vendrá definido por el contexto que determine la selección de uno u otro enunciado. El uso del aspecto permite manifestar, además, que una acción está completamente acabada, incluso si existe una conexión con el tiempo 
presente, como se aprecia en He escrito una monografía sobre los verbos modales en lengua inglesa y me la publican en una editorial londinense.

El modo puede venir recogido mediante elementos morfológicos y sirve para indicar hipótesis en contraposición a un hecho factual en la descripción de una realidad, como se desprende de las palabras de Huddleston (1984: 80):

'Mood' is then used for an inflectional system of the verb where the contrasts between the terms characteristically involve factuality vs nonfactuality, assertions vs nonassertions, main clauses vs subordinate clauses.

Teniendo en cuenta la definición de modalidad expuesta anteriormente, se aprecia la conexión del modo con los verbos modales, con la salvedad de que el primero es un fenómeno gramaticalizado. Decir No haría esa receta sin ayuda no está tan alejado de No puedo hacer esa receta sin ayuda, aunque la perspectiva se conceptualiza morfológicamente en el primer caso y mediante una perífrasis modal en el segundo. Sobre las perífrasis modales, trata la sección siguiente.

\subsubsection{Los verbos modales}

En lengua inglesa, existen nueve verbos modales centrales, como se apunta en Biber y otros (1999: 483), a saber: can, could, may, might, shall, should, will, would y must. Estos autores establecen una relación entre modales formando pares de acuerdo a un valor gramatical basado en la expresión del tiempo: can y could, may y might, shall y should, will y would (1999: 485). Sin embargo, a pesar de este uso con un origen bajomedieval, las formas en pasado de estos verbos tienen diferentes funciones pragmáticas, entre ellas, la mitigación del contenido proposicional como estrategia de cortesía, pues se evita 
imponer el criterio del hablante, como ocurre con could en These results obtained from this study could help us understand the reasons for the lack of visitors in the islands last summer. En este ejemplo, toda la proposición se atenúa por medio del uso del verbo modal que indica probabilidad de que se cumpla lo expresado. Desde luego, could no sería una forma de pasado en este caso. Sí, sería así en el ejemplo The island could host the summer music fest last year, and it was a complete success, donde la forma modal indica tiempo anterior.

A los verbos mencionados, se añaden otros que se denominan modales periféricos o modales marginales, aunque es muy frecuente su denominación como semi-modales, en inglés semi-modals. Estos son los siguientes: need (to), ought to, dare (to), y used to. Como se puede apreciar, uno de los aspectos que destaca en los semi-modales con respecto a los modales centrales es la posibilidad de que los primeros pueden ir seguidos de to más infinitivo, mientras que los segundos pueden ir seguidos de un verbo en infinitivo sin to. Semejantes a los semi-modales se encuentra un tercer grupo de expresiones idiomáticas con significación modal (Biber et al. 1999: 484): have to, had better, be supposed to...

La descripción de las perífrasis modales en lengua inglesa puede ser según criterios morfológicos, sintácticos y semánticos, según Denison (1993: 292 ff), que resultan en los siguientes parámetros:

a. las perífrasis modales en inglés no cuentan con formas finitas y, por lo tanto, no es posible encontrar formas como *to may y *to can, 
b. la distinción temporal puede darse en estas formas, como se ha explicado anteriormente, pero puede que estas expresiones en pasado tengan un significado contextual y no representen tiempo pasado,

c. los verbos modales no presentan desinencia de tercera persona del singular del presente de indicativo, o sea, las formas *she cans, *he mays, *he musts, *she wills no son aceptables,

d. muchos verbos modales presentan formas contraídas en su forma negativa (can't, won't, mustn't), y algunas incluso muestran formas fonológicas reducidas en forma de clítico, como 'll, 'd, en lugar de shall/will y de would, respectivamente,

e. estos verbos no tienen formas de imperativo,

f. los verbos modales van seguido por infinitivos sin to: She may stay,

g. los verbos modales afectan todo el contenido proposicional de la cláusula en la que aparecen: MAY [She stay],

h. desde una perspectiva dialectal, puede darse la circunstancia de que haya más de un verbo modal seguidos, como es el caso de algunas variantes del norte de Inglaterra y de Escocia, donde podemos encontrar caso de doble modal: So / say-you won't can read it lass (ejemplo tomado de Tagliamonte (2013: 24)),

i. como operadores, comparten un conjunto de propiedades conocidas con el acrónimo NICE: (a) se puede negar mediante el uso de $n^{\prime} t /$ not, (b) pueden realizar inversión sujeto-verbo, (c) admiten elisión del contenido proposicional, esto es tiene función coda, como en A: Can you come with me? B: Of course, I can, donde el uso de can en la respuesta permite elidir 
el contenido proposicional, y (d) puede ser usado en cuestiones de polaridad enfática.

\subsubsection{Los mecanismos léxicos}

Una manera de mostrar modalidad es mediante mecanismos léxicos, como apuntan Facchinetti, Krug y Palmer (2003: vi): "Modality is realized by linguistic items from a wide range of grammatical classes, covering not only modal auxiliaries and lexical verbs, but also nouns, adjectives, adverbs, idioms, particles, mood and prosody in speech." Un caso claro de estrategias léxicas para modular el contenido proposicional son lo que se conoce como hedge en inglés en el sentido propuesto en Hyland (1994) y que especifican significado epistémico. Mur-Dueñas (2011: 3070) define este concepto como: "features which limit the writer's full commitment to what is stated in a proposition and which may be the result of certain pragmatic conventions in academic writing." Ejemplos serían el uso de la matriz It is likely that o el adverbio probably que atenúan el contenido de la proposición a la que acompañan.

Huddleston and Pullum (2002: 176) se refieren a estos mecanismos como lexical modals, que ilustran y definen de la siguiente manera:

We use this term for items expressing the same kind of meaning as the modal auxiliaries, but which do not belong to the syntactic class of auxiliary verbs. It covers adjectives like possible, necessary, likely, probable, bound, supposed, adverbs like perhaps, possibly, necessarily, probably, certainly, surely, verbs like insist, permit, require, and nouns like possibility, necessity, permission, and similar derivatives.

En estas palabras, se aprecia que los vocablos con contenido modal se relacionan con los campos semánticos de probabilidad, posibilidad y necesidad. Este último incluye 
significados de obligación y permiso, estos complementarios en tanto que la noción de permiso puede indicar la ausencia de obligación o de prohibición, como en Students are allowed to come in now to the exam theatre. Aquí la noción de permiso se desprende de la ausencia de prohibición de acceso al recinto de examen.

\subsubsection{Objetividad, subjetividad e intersubjetividad}

La modalidad se entiende como un fenómeno subjetivo, como se apunta en Hyland (1997, 1994, 2002) y Carrió-Pastor (2014) y Carrió-Pastor y Calderón (2015), entre otros. Sin embargo, el lenguaje científico siempre se ha caracterizado por pretender una comunicación objetiva de los avances que se han llevado a cabo en un campo específico. Sin embargo, la propia concepción de objetividad implica la idea de subjetividad, en tanto que una comunicación objetiva implicaría la ausencia de un conceptualizador que sería responsable de la formulación del contenido, como se explica también en lo siguiente:

It has been argued that, in the objective point of view, the speaker or the writer intends (or pretends) to describe things as if there were no subject of consciousness behind the utterance (Almeida \& Ferrari 2010: 112).

Taylor y Littlemore (2014: 12) exponen mediante el ejemplo The cat is sitting on the mat que la objetividad plantea una serie de problemas, pues cuáles son exactamente las referencias que un determinado hablante hace cuando usa los términos cat y mat. ¿Se trata, por ejemplo, en el caso de mat, de un tipo de alfombra o, mejor, de un tipo de felpudo? Cuando se usa la preposición on para hablar sobre una ubicación, esta puede indicar diferentes posiciones en las que se encontraría el gato. Con esto, se evidencia que la objetividad parece difícil de comunicar en tanto que existen grados de adaptación de 
los significados a los contextos personales, así como grados de perspectivización del contenido para ajustarse a las necesidades comunicativas del propio hablante.

De lo expuesto anteriormente, se deduce que existe subjetividad en las expresiones manifestadas por los hablantes mediante la cual se manifiesta un punto de vista determinado, como se aprecia en This year the English team could be the winner. La diferencia entre The cat is sitting on the mat y el último ejemplo es el uso de la partícula modal could, por la que la visión subjetiva del hablante queda explícita y codificada lingüísticamente. Se expresa incertidumbre mediante la manifestación de una hipótesis que es probable que ocurra en el futuro.

Lyons (1994: 13) resume la subjetividad en estos términos:

[...] locutionary subjectivity is the locutionary agent's (the speaker's or writer's, the utterer's) expression of himself or herself in the act of utterance: locutionary subjectivity is, quite simply, self-expression in the use of language.

Aquí queda patente el papel que juega el hablante y la expresión de su punto de vista en la formulación del contenido proposicional. En la gramática cognitiva de Langacker (1999; 2009), la subjetividad coincide exactamente con la perspectiva acerca de la [re]construcción del escenario en la que se desarrolla el evento al que se alude, en la que el conceptualizador observa off-stage la realidad descrita, por la que ha de ser construida subjetivamente.

Por otra parte, la confluencia de varios sistemas cognitivos responde a una interpretación intersubjetiva de la información aportada. Zlatev y otros (2008: 1) definen la intersubjetividad como "the sharing of experiential content (e.g., feelings, perceptions, 
thoughts, and linguistic meanings) among a plurality of subjects" (el énfasis procede del original). Por lo tanto, la información intersubjetiva procede de la concurrencia de la perspectiva que emana de los diferentes participantes en el intercambio comunicativo:

Just as all language use can be said to be subjective in a general sense, it is intersubjective in a general sense, reflecting the impact of the speech situation which not just involves a speaker but a communicative relationship between speaker and hearer (Cuyckens, Davidse \& Vandelanotte 2010: 14).

En este trabajo, estos conceptos, en especial la subjetividad y la intersubjetividad, son fundamentales en la interpretación de los empleos que se hace de los verbos modales, pues la expresión autónoma o colegiada del punto de vista tiene una repercusión en los efectos pragmáticos para indicar, entre otros aspectos, grados de cortesía lingüística (mediante la mitigación del contenido proposicional) o la expresión de autoridad mediante el refuerzo traído por la recurrencia en el punto de vista.

Dado que esta tesis abarca un análisis de las perífrasis modales en el artículo científico en el registro turístico es pertinente una descripción del género para poder contextualizar el análisis que se lleve a cabo de las perífrasis en la siguiente sección.

\subsection{El artículo de investigación en turismo}

Como se ha dicho, en esta sección, se pretende dar cuenta de la forma que presenta el artículo de investigación en turismo. A pesar de lo que pueda parecer por la importancia que pueda tener la investigación en turismo para las economías mundiales, el género no ha gozado de gran atención en los estudios de crítica textual desde el punto de vista del género, como señalan Lin y Evans (2012). Antes de abordar la estructura del artículo, es necesario una contextualización de los fundamentos teóricos, siguiendo la teoría 
sistémico-funcional. Esta descripción es importante para la identificación correcta de las secciones correspondientes a la introducción y a la conclusión de los artículos, que se explicará en el capítulo 3, pues forman parte del corpus de trabaja para el análisis de las formas modales.

\subsubsection{Tipo de texto, género, registro en el contexto de la teoría sistémico-} funcional (TSF). Descripción de conceptos

Los tipos de texto, el género y el registro se han estudiado en el marco de la lingüística funcional y sistémico-funcional, como se puede apreciar en los trabajos de Martin (1984), Halliday and Hasan (1985) y Moessner (2001), entre otros. Estas teorías se han aplicado a estudios de géneros de escritura académica y profesional (cf. Swales 1990; Bhatia 1993; Azar 2012; 2013; 2014; 2015). La definición del primer concepto mencionado arriba, los tipos de textos, depende del enfoque (funcional, estructural, social, psicológico, etc.) que se use para precisarlo. En este trabajo, se usará una aproximación funcional, por lo que, siguiendo a Biber (1988: 70), los tipos de textos se refieren a "groupings of texts that are similar with respect to their linguistic form, irrespective of genre categories". Biber y Finegan (1989), de acuerdo con Werlich (1976), identifican los siguientes tipos textuales: descriptivo, narrativo, expositivo, argumentativo e instructivo. Los tipos de textos proporcionan información acerca de las estructuras lingüísticas asociadas a cada uno de ellos. Biber (1988) relaciona una serie de estas estructuras que funcionan como marcadores de los tipos textuales. Algunas de estas son las formas nominales, los adverbios de tiempo, las proformas, los adjetivos, la especificidad léxica, las perífrasis modales, las formas nominales, los verbos léxicos y los adverbios, entre otras estructuras ya sean morfológicas, sintácticas o léxicas. 
Puesto que se trata de elementos lingüísticos formales, su uso estará en relación directa con el tipo de texto que se trata. De ahí que un texto narrativo tenga como característica principal, por ejemplo, un uso específico de los tiempos verbales en pasado o en presente, según se la perspectiva que se pretenda en la narración. En general, diremos que un determinado conjunto de características es propio de uno u otro tipo de texto y, por lo tanto, lo caracterizan. Obviamente, esto tiene unas implicaciones pedagógicas relevantes para el aprendizaje de lenguas, pero también para el aprendizaje de lenguas de especialidad y, en concreto, para el relativo a la escritura académica. Podríamos decir que, en el caso del artículo de investigación en turismo como este se compone de algunos tipos de textos dependiendo de la intencionalidad del autor en cada momento de su trabajo, las perífrasis modales en principio serían una característica formal de la argumentación que se establecería tanto en la discusión de los datos, como en la conclusión general al trabajo que se presenta, como veremos en el capítulo siguiente.

Otro ejemplo de este tipo de artículos, y como apuntan Alonso-Almeida y Álvarez-Gil (2021a), es el uso del pasado para expresar objetividad que ocurre en los textos narrativos que se incluyen en la descripción de la recogida de datos, donde se ofrece con gran detalle relatos episódicos de cómo se ha llevado a cabo dicho proceso de la investigación. Esto se aprecia en el siguiente ejemplo tomado de un artículo de investigación en turismo que se ha usado para extraer las introducciones y las conclusiones del corpus de trabajo. Aquí, se aprecia el "narrative style" que comentan Alonso-Almeida y Álvarez-Gil (2021a) y que mencionan como caracterizador del artículo en turismo, como se explicará en la sección siguiente (las negritas en este ejemplo son editoriales). Aquí se puede observar de forma precisa el uso del pasado para relatar el procedimiento de método que se ha usado de manera muy explícita frente a la forma 
más condensada que suelen presentar los artículos de investigación en lingüística, por ejemplo. En este mismo ejemplo, el uso de presente se intercala exclusivamente para señalar una característica propia o una generalidad, como ocurre en "Romanians are not generally comfortable with being recorded" en este ejemplo.

Interviewing was undertaken in Romanian by two 'teams': the first comprised two of the authors (a Romanian woman and a British man). Both were Romanian speakers and both were broadly similar in age to the research participants. Moreover, having lived through the socialist period, the Romanian researcher had shared the broader experiences of the interviewees. The second team comprised a young, male Romanian research assistant and one of the authors (a British man who does not speak Romanian). Feminist/ gender research stresses reflexivity on the part of the researcher (Pritchard, 2014; Tickner, 2005) and we must recognise our positionality in relation to our research participants. In particular, the women that we spoke to may have been uncomfortable about discussing their experiences of working in tourism with strangers, some of whom were both men, and not Romanian. However, most proved very willing to talk about working in tourism under socialism. Indeed, they were visibly more comfortable when it was apparent that we wished to discuss the socialist past rather than the contemporary state of tourism at the Black Sea. Only one issue - surveillance by the state authorities - provoked discomfort in some participants, and we did not pursue this issue in such cases. The interviews lasted between 20 and 45 minutes and, since Romanians are not generally comfortable with being recorded, we made notes which were subsequently written up into a more detailed account. These were then analysed using standard qualitative coding methods (Dumbrăveanu et al. 2016: 156157).

En cuanto al género textual, este presenta una dimensión social y funcional, en tanto que un género tiene una misión concreta. Una nota tiene como misión dejar un mensaje rápido a alguien o a uno/a mismo/a. Un manual de instrucciones tiene como objetivo instruir sobre cómo se ha de hacer algo. Los hablantes de una determinada lengua son capaces de reconocer estos hechos lingüísticos e interpretarlos. Existen muchas 
definiciones de género, si bien todas señalan este aspecto social y comunicativo de los géneros textuales, como se puede ver en la aportada por Biber (1988: 170) cuando dice que las categorías de géneros "are determined on the basis of external criteria relating to the speaker's purpose and topic; they are assigned on the basis of use rather than on the basis of form". De aquí se deduce que la intencionalidad es fundamental en la configuración de un género y también el tema que se trate. Sin embargo, el tema no está más relacionado para algunos autores (cf. Alonso-Almeida 2008) con el concepto de registro, que veremos más adelante, que con el de género textual. En efecto, el propio artículo de investigación es un género independientemente de la disciplina que abarque, pues el género tiene entre sus peculiaridades marcar la estructura informativa en la que se desarrolla y debiera influir en las cuestiones léxicas que pueden ser más propias del tipo de texto o, mejor, del registro, como se ha apuntado.

La funcionalidad del género, el propósito, la dimensión social y la estructura son también parte de la definición de este concepto que propone Swales (1990):

Genre is a class of communicative events, the members of which share some set of communicative purposes. These purposes are recognized by the expert members of the parent discourse community, and thereby constitute the rationale of the genre. This rationale shapes the schematic structure of the discourse and influences and constrains choices of content and style. Communicative purpose is both a privileged criterion and one that operates to keep the scope of a genre as here conceived narrowly focused on comparable rhetorical action. In addition to purpose, exemplars of a genre exhibit various patterns of similarity in terms of structure, style, content and intended audience (Swales 1990: 58).

Para este autor, la estructura de los géneros se configura de acuerdo a una serie de moves y steps, siguiendo la terminología de Swales (1990). En cuanto a Martin (1984: 25), usa el 
témino stage en su definición de género textual: "a staged, goal-oriented, purposeful activity in which speakers engage as members of our culture". Traduciremos el término "stage" en este trabajo como "sección" por ser un término ampliamente usado en la literatura de los géneros textuales y, particularmente, en lo relativo a los artículos científicos. Eggins (1994: 41) establece dos tipos de estructuras descriptivas del género según el concepto de sección (o stage). La primera de ellas se denomina potencial de estructura de un género (generic structure potential), que incluye todas las secciones posibles que se pueden encontrar en un género textual $y$, con frecuencia, se indica el posible orden de estas mediante un circunflejo para señalar el orden fijo de una sección en la secuencia dada o un asterisco para indicar la existencia de un orden aleatorio de una determinada sección con respecto al resto en la secuencia; el uso de paréntesis significa que una sección es opcional. La segunda es la estructura genérica real (actual generic structure) y se refiere a la secuencia de secciones de un ejemplo de género concreto. En el caso de los artículos de investigación, la estructura que presenta un artículo es su estructura genérica real y la suma de las estructuras genéricas reales de varios artículos dan como resultado el potencial de estructura de un género.

Los géneros pueden presentar diferencias en cuanto al registro, como puede ser el tema o disciplina sobre el que se escriba. Así, los artículos pueden versar sobre ingeniería, geografía, medicina, historia, psicología o lingüística. Sin embargo, como se apuntará más adelante siguiendo los resultados sobre el estudio del género artículo de investigación en la disciplina de turismo en Alonso-Almeida y Álvarez-Gil (2021a), la variable "disciplina" puede justificar variaciones en cuanto a la presencia de las secciones en un artículo y también en cuanto al uso de la lengua. En lingüística sistémico-funcional, el término registro se define de la siguiente manera: 
A register is a functional variety of language (Halliday, McIntosh \& Strevens 1964; Halliday 1978) - the patterns of instantiation of the overall system associated with a given type of context (a situation type). 8 These patterns of instantiation show up quantitatively as adjustments in the systemic probabilities of language; a register can be represented as a particular setting of systemic probabilities. For example, the future tense is very much more likely to occur in weather forecasts than it is in stories (Halliday \& Matthiessen 2014: 29).

El registro se relaciona con tres metafunciones, según Halliday (1985a), que son la ideacional, la interpersonal y la textual. La metafunción ideacional tiene en cuenta la expresión de las experiencias y el contenido. La metafunción interpersonal concierne la manera en la que se llevan a cabo las acciones y la relación que existe entre los participantes en un intercambio comunicativo. Finalmente, la metafunción textual se refiere a la construcción del texto y las relaciones entre las partes del entramado textual. Las variables del registro son tres (Halliday 1985b): campo, modo y tenor. Flowerdew (2013: 12-13) define estos conceptos de la lingüística sistémica de la siguiente manera:

(a) campo: "the purpose of the communication and what it is about";

(b) modo: "how the language is organised and functions in the interaction, for example, whether it is written or spoken or some combination of the two (as in various electronic modalities, whether it is expository, or didactic or persuasive, and so forth"; y

(c) tenor: "the relations between the participants in the text".

El campo está relacionado con la metafunción ideacional, el modo con la con la textual y el tenor con la interpersonal, lo que se resuelve en una serie de decisiones léxicas y gramaticales en la producción de un texto. 


\subsubsection{El artículo de investigación en turismo. Estructura del artículo}

El artículo científico constituye un género textual en tanto que presenta una estructura concreta, reconocible por la comunidad de científicos, y que cumple una función específica. Swales (1990: 93) define este género como:

a written text (although often containing non-verbal elements), usually limited to a few thousand words, that reports on some investigation carried out by its author or authors. In addition, the RA will usually relate the findings within it to those of others, and may also examine issues of theory and/or methodology. It is to appear or has appeared in a research journal or, less typically, in an edited book-length collection of papers.

Siguiendo el modelo funcionalista, el artículo de investigación en turismo publicados en revistas científicas tiene como campo de estudio hechos relativos al turismo y a la hostelería. El tenor se compone de los propios investigadores, otros investigadores, estudiantes, empresarios y políticos, todos ellos con un afán de estudio y/o de mejora de la actividad en el sector. Por último, el modo es escrito en publicaciones en formato papel y/o electrónico.

La forma genérica del artículo de investigación en turismo no ha recibido mucha atención por parte de la crítica científica, como se apunta en Lin y Evans (2012) quienes argumentan la alta variabilidad estructural del discurso científico como uno de los principales escollos con los que los investigadores se encuentran para poder definir con precisión el género en este registro. Alonso-Almeida y Álvarez-Gil (2021a) han tratado de desvelar el formato del artículo de investigación turístico mediante el análisis de las estructuras reales de géneros de 74 artículos, todos ellos tomados de revistas de impacto 
en la materia y será este trabajo el que me servirá de modelo para describir el género aquí.

Aunque estos autores también encontraron gran variación en el uso, aparición y distribución de las secciones, su propuesta de potencial genérico incluye 7 de éstas. Este patrón organizativo presenta una única sección que siempre aparece, esto es la introducción, mientras que el resto son opcionales, bien por no aparecer, bien por estar subsumidas dentro de otras sin que exista la posibilidad de segmentarlas. Así, el potencial quedaría de la siguiente manera usando la terminología inglesa usada por los autores, pero que traducimos en la figura 1:

Introducción ^ (Enfoque $)^{\wedge}(\text { Diseño de la investigación })^{\wedge}(\text { Resultados })^{\wedge}(\text { Discusión })^{\wedge}$ (Conclusión)^ (Epílogo)

Figura 1. Potencial genérico del artículo de investigación en turismo, según Alonso-Almeida y ÁlvarezGil (2021a).

En este estudio, encontraron que todos los textos presentaban obligatoriamente una introducción, si bien no todas señalaban este hecho con un título de sección acorde a su contenido. Las siguientes secciones con mayor presencia fueron la approach, conclusion, y la discussion, en este orden. La sección results y design aparecen en menor proporción, menos de la mitad de las veces. La presencia de la sección denominada postlude se constata de manera anecdótica, pues solo se presentaba en uno de los artículos, pero con una función muy precisa, por lo que los investigadores decidieron mantenerla. En el gráfico 1 (adaptado de Alonso-Almeida y Álvarez-Gil, 2021a), se ofrece la distribución encontrada en el estudio mencionado y que se reproduce en el siguiente gráfico: 


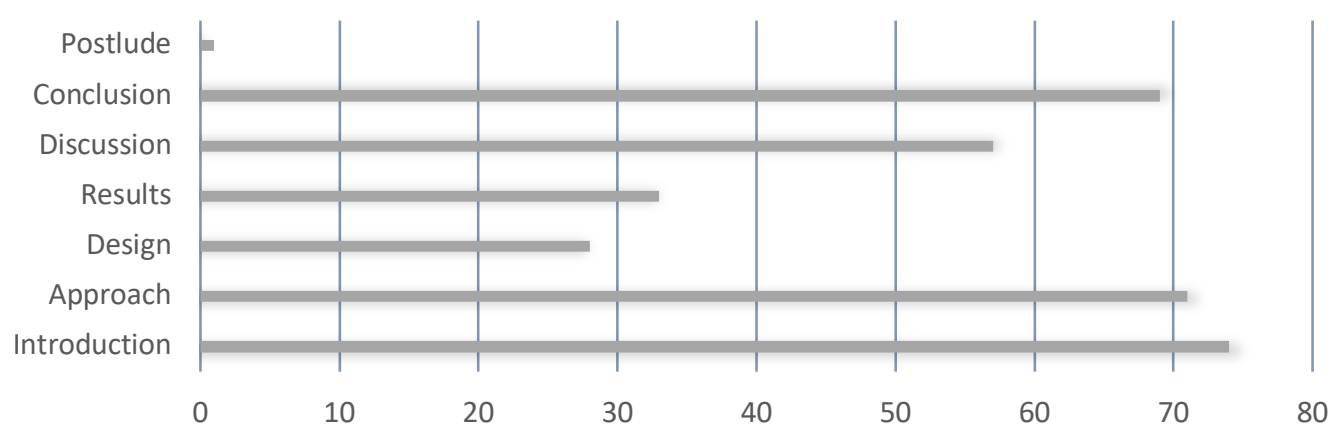

Gráfico 1. Secciones de potencial genérico y distribución, adaptado de Alonso-Almeida y Álvarez-Gil (2021a).

Las funciones de estas secciones fueron descritas en Alonso-Almeida y Álvarez-Gil (2021a). Así, la introducción (introduction), se usa para (a) exponer los motivos y justificación que conducen al estudio que se plantea, (b) indicar los objetivos, (c) plantear la hipótesis de estudio, y (d) exponer los fundamentos teóricos y los métodos que han empleado. El enfoque (approach) tiene las funciones de (a) describir los antecedentes teóricos de la investigación llevada a cabo y (b) describir la actividad de una investigación similar en turismo. En cuanto a la sección diseño de la investigación (research design) se trata aquí de describir cómo se ha llevado a cabo la actividad de investigación. En cuanto a los resultados (results), se persigue en esta sección resumir las principales conclusiones del análisis realizado sobre un conjunto de datos.

La sección discusión (discussion) tiene como objetivo (a) interpretar los posibles significados de los datos obtenidos a partir del análisis de los resultados en el contexto del marco teórico, (b) aportar inferencias a la luz de los resultados, (c) ofrecer observaciones finales, "especially in cases where a proper conclusion stage is not included", como apuntan Alonso-Almeida y Álvarez-Gil (2021a). En el caso de la 
conclusión (conclusión), su funciones son (a) describir la(s) principal(es) contribución(es) del trabajo, (b) mostrar la perspectiva del autor con respecto a los datos observados y dentro del conocimiento existente, (c) identificar los puntos fuertes y las deficiencias de la investigación que se presenta, (d) esbozar orientaciones/afirmaciones para futuras investigaciones, y (e) el epílogo (postlude), aunque solo se da un caso, según los autores, su función principal es la de ofrecer información relevante e inesperada obtenida tras la finalización del trabajo y de la investigación que se discute.

Algunas secciones se indican claramente mediante títulos que presentan ciertas coincidencias con los que usan Alonso-Almeida y Álvarez-Gil (2021a) para nombrar las secciones. Sin embargo, como se apunta en ese mismo trabajo, el artículo de investigación en turismo no siempre usa secciones que indican claramente la función que representan, bien al contrario "some section headings in some of the examined cases may describe descriptive of the content to the extent that headings become very creative and original". Algunos ejemplos en este sentido son los siguientes para la sección discusión, que indican la variación existente para una misma sección, ya sea incluyendo o no la palabra "sección", como en los casos siguientes tomados de artículos de investigación en turismo:

The familiar foreign (Tchoukarine 2016: 393).

Discussion: the gaze as arbiter in a 'liquid modern world' (Hocking 2016: 376).

Residents' attitudes towards Chinese tourists in Niseko (Nelson \& Matthews 2018: 224).

Como se ha indicado anteriormente, las secciones de un artículo de investigación no siempre son fáciles de distinguir, pues depende de los autores y las divisiones que 
consideren oportunas, a menos de que exista una política editorial rígida que exija una estructura concreta del artículo. Una de las maneras en las que Alonso-Almeida y ÁlvarezGil (2021a) justifican y reconocen las secciones genéricas es mediante el uso de patrones léxicos y morfológicos concretos, que se explican en la siguiente sección con ejemplos tomados de los mismos artículos usados para formar el corpus de introducciones y conclusiones y cuyas referencias se encuentran en el apéndice de este trabajo.

\subsection{Lenguaje y secciones del artículo de investigación}

Para caracterizar e identificar las secciones que forman parte del corpus compilado se aplicarán las fórmulas léxicas y/o gramaticales que Alonso-Almeida y Álvarez-Gil (2021a: 15) han registrado como distintivas para cada sección del artículo de investigación, si bien esto solo representa una parte muy pequeña en la descripción del género realizada por estos autores. Con esta prueba, se pretende confirmar fuera de toda duda la ideoneidad de los textos seleccionados como introducciones y conclusiones. Así, los ejemplos correspondientes a las introducciones y las conclusiones en esta sección son del corpus, por lo que se destacan mediante numeración consecutiva, como se ha explicado anteriormente. Con esto, se distinguen de aquellos que sirven para ilustrar las otras secciones y que se toman de los mismos artículos de donde se han extractado las introducciones y las conclusiones, pero que no forman parte de la compilación textual usada en el análisis de las perífrasis modales. Con todo, se consigue una caracterización en cuanto a la forma del artículo de investigación según el uso de la lengua, además de garantizar que los textos incluidos como introducciones y conclusiones en la compilación realizada sean tales. 
Muchas de las fórmulas que se han identificado como caracterizadoras en el género del artículo de investigación en turismo reflejan con claridad la función de las secciones que lo componen. En la introducción, por ejemplo, se encuentran fórmulas que manifiestan el objetivo del artículo de investigación, como (a) The (ADJ) (aim/purpose/point) of (the/this article/study) is to INF, como se muestra en los siguientes ejemplos y (b) This article's central purpose is to INF.

a) The aim of this article is to present a case study scenario for the future of hotels in larger cities that serve this new community of global citizens (Lub et al. 2016).

The key purpose of the research is to provide a tool to better explain consumers' intention to book tourism products online (Sahli y Legohérel 2016).

The purpose of the article is to examine how theoretical understandings of authenticity, staging, and negotiation play out in on-line communities of travel reviewers who have reported on their touristic experiences of "hill tribe" attractions in northern Thailand (Walter 2016).

The purpose of this study is thus to analyze the patterns of household expenditure on tourism in different life stages using the national household survey data for China (Lin et. 2015).

By analysing the narratives of individuals undertaking a geotour, the aim of this article is to explore the experiential dimension of geotourism (Aquino, Schänzel \& Hyde 2018).

b) This article's central purpose is to extend conceptualizations of existential authenticity by showcasing its scalar dimensions (Gillen 2016).

Existen otras expresiones en la introducción relacionadas con la presentación del tema o de los objetivos, pero estas contienen un verbo léxico comunicativo del tipo SPEAKING, o bien, experiencial del tipo LOOK en la clasificación de Dixon (2005), que siguen AlonsoAlmeida y Álvarez-Gil (2021a). Estas expresiones son The/This article focuses on..., The article has $X$ goals..., This/ Our article addresses..., The article presents...: 
This study focuses on music-making on the island, examining ways in which resident musicians negotiate the particular set of circumstances created by Fernando de Noronha's reliance on the tourist economy (Fitzgerald \& Reis 2016).

The investigation focuses on the ways the tourist experience is communicated in this particular context (Wang y Alasuutari 2017).

Our article addresses a significant issue in tourism policy that connects to the nature and future of its markets (Taznelli y Korstanje 2016).

Subsequently, the article presents the main results and conclusions and gives suggestions for a future research agenda (Hernmann et al. 2017).

This article thus addresses the increasingly important role of social media in creating and changing the identity of place and person, while illustrating the power of discourse in the maintenance of place identity (Weatherby \& Vidon 2018).

Therefore, this article presents a comparative qualitative empirical perspective, exploring how, and in which ways, tourists involve this kind of identity-work in practice during their visits to music-related locations (Bolderman \& Reijnders 2017).

Igualmente, se usan expresiones con verbos del tipo discuss, conclude, y report (Dixon 2005) en las fórmulas This article argues/explores/ examines/shows/demonstrates..., (In this article,) we argue/ approach/ explore/ focus (ADV) on/ (shall) examine/ theorise/ demonstrate..., This/The article draws on... y This/The article proposes/concludes... Todas estas se relacionan con la intención de mostrar y marcar la naturaleza empírica del artículo de investigación, así como los procesos cognitivos que ocurren en la argumentación, como se muestra en los siguientes ejemplos:

This article explores some of these effects, as identified by Niseko residents, and for reasons outlined below unpacks their responses to two specific tourist groups: those from Australia and China (Nelson \& Matthews 2018).

In this article, we shall examine what kind of a gateway Iceland represents by focusing on how it is promoted as a tourism destination aimed at stopover visitors... (Lund, Loftsdóttir \& Leonard 2017).

By investigating one popular rock climbing destination-Red River Gorge, Kentucky, USA - this article demonstrates that mobilities invite encounters with and enactments of place such that travel rhythms, everyday rhythms, and natural rhythms coalesce, interrupt, and even emerge anew (Rickly 2017a). 
The article first draws on and engages with the theoretical background, then provides the methodological approach and empirical analysis of the study, and concludes with discussion and further research (Bui y Wilkins 2017).

We argue that locally situated responses to globally imposed troika policies (see Robertson, 1995 on 'glocalisation'), point to a radical alteration in the country's image as a tourist destination (Taznelli y Korstanje 2016).

The paper concludes with a discussion of the implications of the findings for management practice and future research (Song et al. 2018).

Finalmente, en esta sección, se emplean una serie de fórmulas léxico-gramaticales que hacen referencia a la estructura del artículo de investigación, que son The article is structured..., The article has $X$ (parts/sections) y The article is organised... Algunos ejemplos de estas estructuras son los siguientes:

The article is organised as follows (Liston-Heyes \& Daley 2017).

This article has four parts (Torabian \& Mair 2017).

This manuscript is structured as follows (Rojas-Méndez and Michael J Hine 2017).

This article has seven sections including the previous introduction (Nilsson y Tesfahuney 2018).

En el enfoque, las fórmulas usadas contienen, como apuntan Alonso-Almeida y ÁlvarezGil (2021a: 20):

a number of impersonal matrices containing effective or epistemic nuances (cf. MarínArrese 2009) that signal epistemological information along with the authors' stance towards the information that they either want to highlight... or provide epistemic justification for... using the matrix 'it is evident that...'.

Estas son: It is necessary to... (clarify/ interact/ differentiate/ develop/ use), It should be noted that ATTRIBUTION/EVIDENTIAL, It is evident that..., como se ejemplifica a continuación. 
Before considering the particularities of tourism in Niseko though, it is necessary to develop a clearer understanding of the 'people impacts' of tourism (Wolf, 1977) and the more recent research field of resident attitudes to tourism (Nelson \& Matthews 2018: 216).

However, it should be noted some tourism scholars have explored these issues, and their work helps to set the stage for more critical investigations of travel policies and their implications for mobility (Torabian \& Mair 2017: 20).

It is evident that the microeconomic modeling of tourism expenditure has been dominated by researchers from developed countries/regions, with a very strong concentration in the USA and the UK (Lin et al. 2017: 102).

Otras expresiones en esta sección se usan para hacer referencia al campo de estudio o, incluso a las asociaciones entre disciplinas o aspectos que deban tenerse en cuenta en la investigación. Estas expresiones son ...in DET (ADJ) field of (inquiry/tourism/research)... y ...the relationship between (FIELD/ITEM) and (FIELD/ITEM)..., como se ve en los siguientes ejemplos:

Destination image has been studied in the field of tourism for more than 35 years (Salvatierra \& Walters 2015: 74).

Perhaps, the most significant theoretical evolution in the field of heritage studies is the recognition that heritage is fundamentally a dynamic and empowering process (Lundberg, Ziakas \& Morgan 2018: 92).

Few studies have looked into the relationship between accommodation space and family group dynamics, and spatiality, that is, lived and felt space (Van Manen, 1990) as a theme is absent in family tourism research (Schänzel \& Lynch 2016: 137).

Al tratarse de una exposición y descripción del posicionamiento teórico, en esta sección, no es extraño encontrarse definiciones de términos que se introducen mediante la fórmula $X(B E)$ defined (as/in), como se ha indentificado en el corpus de la tesis:

Kim, Ritchie, and McCormick (2012) proposed that a memorable tourism experience has been operationally defined as a tourism experience positively remembered and recalled after the event has occurred (Lee 2015: 159). 
For philosophers, the notion of luck has been defined in various ways which presume three conditions (Zerva 2018: 234).

La sección diseño de la investigación, como señalan Alonso-Almeida y Álvarez-Gil (2021a), presenta expresiones que comprenden sustantivos relativos al método como analysis, data, interview, method y verbos que se refieren a procesos de investigación como conducted, designed, performed, focused on, carried out y transcribed. La premodificación adjetiva del nombre data presenta lexías como anonymous, digital, empirical, formal, incomplete, initial, multimodal, qualitative, rich, statistical, visual y ethnographic, como en los ejemplos siguientes:

The empirical data were collected from consumers at different spa hotels in four major spa regions of Taiwan during July-August 2013 (Shiu 2018: 31).

The statistical data for the micro-level analysis were derived from the annual reports of the studied hotel chains for the year 2010 (Mendieta-Peñalver et al. 2018: 14).

Through visits to 53 hotels, we conducted interviews with 19 people (15 women and 4 men) who had worked in a wide range of positions (from cleaner to manager) (Dumbrăveanu et al. 2016: 156).

However, it is important to note that concurrent general branding images were also analysed providing the context for the more directed MyStopover campaign; these images were obtained from standard desktop research using an Internet search that focused specifically on Icelandair's main website (Lund, Loftsdóttir \& Leonard 2017: 151).

Se aprecia también recurrencia de la fórmula léxico-gramatical (DET) interviews PASSIVE con las formas del verbo en pasado:

First, the interviews were transcribed verbatim and consequently analysed using Atlas.ti (Bolderman \& Reijnders 2017: 170).

All the interviews were carried out personally, either face-to-face or on the phone, and lasted approximately 15-20 minutes (Beritelli \& Reinhold 2018: 432). 
La sección de resultados muestra léxico asociado a verbos relacionados con los campos semánticos "mostrar" y "descubrir", para evidenciar la naturaleza empírica de la investigación. En este sentido, se encontró en el estudio mencionado arriba los patrones siguientes que contienen verbos como reveal, show, suggest, yield, demonstrate, illustrate, plot, summarize y present, entre otros:

(DET) results (reveal(ed)/ show/(ed)/ suggest/ yield)...: Regarding to motivation and memorable experiences, the results reveal parameters for the path between personal emotion and memorable experiences $(b=.14, p=.05)$ and for that between knowledge learning and memorable experiences $(b=.08, p=.22) \ldots$ The results revealed significantly positive relationships between personal emotion and memorable experiences as well as between cultural inheritance and memorable experiences (Lee 2017: 165).

(DET) findings (suggest/ demonstrate/ show/ lend support/ illustrate/ indicate/ emphasise)...: Indeed, our findings lend support to the argument that gap year experiences are foremost represented as tools for individualised identity work (Hermann, Peters \& Van Trijp 2017: 87).

Results (are/were)(presented/summarized/plotted)...: The results are plotted in Fig. 8 (Lin et al. 2015: 111).

(DET) (PREMODIFICATION) results (are) presented in Table $X$ : ...the CFA results are presented in Table 2 (Park, Seo \& Kandampully 2016: 313).

(Fig. X/ Table X) shows that...: Fig. 10 shows that before the age of 25, people with different educational attainment levels tended to have very similar total tourism expenditure (Lin et al. 2015: 113).

As shown/illustrated in Table X... $(\leftarrow$ Adverbial): This international growth, as illustrated in Figure 4 , has been supported by much more flexible expansion formulas (involving management and franchise contracts) and on investments that require a greater level of financial risk (Mendieta-Peñalver et al. 2018: 20). 
Las estructuras que aparecen en la sección discusión se relacionan con la expresión de la perspectiva. Así, se usan fórmulas de significado epistémico, como ...NP (would seem/ seems) (that/to)..., o efectivas, según la terminología en Marín Arrese (2009), para indicar necesidad, como ...it is important to $V$....

There is no way to provide definitive answers to any of these questions, only to stress that such commitment requires time and effort and seems to currently help in small ways (Tzanelli \& Korstanje 2016: 306).

It is important to stress that the images of the workers have a very low level of sexualisation (Vanolo \& Cattan 2017: 417).

Otras fórmulas en esta sección están relacionadas con la presentación de hechos, la atribución, la ejemplificación y añadidos, entre otros aspectos, como se aprecia en los siguientes ejemplos:

The fact that...: The fact that chance meetings happen despite ample space to miss and despite travelers' apparent freewill to move about a destination space is a result of common decision heuristics (Beritelli \& Reinhold 2018: 436).

....according to $(D E T)(A D J / N)$ (terms, analysis, survey, the work of...): As discussed previously, according to feminist critical literature (see for example, Arend, 2014), the masculine gaze operates by transforming feminine subjects into kinds of sexualised objects to be observed and visually consumed, specifically through the gazes of the photographer and the reader (Vanolo \& Cattan 2017: 415).

....as (is/was/in) the case (of/in/with)...: Demonstrations of tourism-cultural capital, such as commenting on the range of interest towards the programme among group members, being attentive towards younger tour participants or, as was the case with the second tour, closing down the questions from over-enthusiastic fans, are all readable as institutionally located strategies (Garner 2017: 438).

...as well as...: The social role attached to backpacker travel as well as their frequent status as single long-term travellers led to often very long interactions (Reichenberger 2017: 272).

...in other words...: Moreover, this obligation of hospitality comes with an expectation of reciprocity when one is traveling away from their "home" area. In other words, mobilizing hospitality among the rock climbing community depends on reciprocity (Rickly 2017b: 67). 
En el caso de la conclusión, se encuentran expresiones con verbos que se refieren a las clases semánticas DISCUSS, CONCLUDE y REPORT (Dixon 2005). Es común en esta sección el uso combinado de los vocablos article, study y research (o la proforma it). Las fórmulas identificadas en esta sección son las siguientes:

...in this article I/we have (examined/presented/ sought/ developed/ implied/ deliberated)...: In this article, we have sought to advance this interdisciplinary agenda by integrating cultural theory and marketing strategy in our exploration of the complex relationship between on-screen popular culture and tourism destination place-making (Lundberg, Ziakas \& Morgan 2018).

In this article, we have deliberated on a new category of pilgrim tourist and some of its key attributes (Nilsson y Tesfahuney 2018).

In this article, we have examined the representation of the gap year product in the Netherlands through a content analysis of Dutch provider websites that link their activities to the gap year experience (Hernmann et al. 2017).

...this article (provides/ reveals/ proposes/ presents/ shows/ suggests/etc.). In unearthing the geotourism experience, this article conceptualises that geotourists' interaction with geology and landscapes is mediated by the multiple aspects of the 'gaze', enriched by their psychological responses and internalisations. This article proposes that geotourism resources at Mount Pinatubo are composed of its natural and cultural landscapes, and tourism environment which visitors have to negotiate with during their journey at the geosite (Aquino, Schänzel \& Hyde 2018).

So beyond segmenting the food lover market by demographics, which has been the focus of much research to date. e.g. Lang Research.Inc. 2001; Sparks et al., 2005; TIAA, 2006), this study reveals that nationalities seek different experiences. (see Molz, 2007) (Andresson et al. 2016).

...it could be (argued/addressed/assumed)...: It could be argued that the short duration of a tourist visit or of the contact in situ with these attractions did not generate important emotional capital, but the latter was produced after their loss, through the re-interpretation of their meanings and values (Zerva 2018).

...it/we (was/has been/have) found (that/to)...: The situational features (Argyle et al., 1981) have been found to be a useful framework to analyse the social interactions of backpackers in New Zealand in more depth and contribute to a greater understanding of how the social aspect of backpacking manifests itself and how it is perceived by participants... However, these aspects are only part of what constitutes backpacker interactions, as their personality type and relationship with the interaction partner have been found to be the core determinants of the impact that these interactions have on their travel experience (Reichenberger 2017). 
Finalmente, en esta sección, se usa con frecuencia la estructura epistémica BE likely to + $\checkmark$ para mitigar el contenido de la proposición que se presenta, como se percibe en los ejemplos siguientes:

Tourists are less likely to rehearse the scenes of Anne's later exploits not only because Anne grows up, but because Anne becomes a local (Gothie 2016).

In conclusion, this study demonstrates that image perceptions towards natural tourism icons such as the GBR are likely to change in response to human-induced environmental damage (Salvatierra \& Walters 2015).

Tourists are likely to choose a destination that aligns with their motives (Peter y Anandkumar 2016).

In the second stage, when travelers are likely to be in an exploratory, informationseeking mode they are open to suggestions to try new things... Furthermore, tourists have not fully scheduled activities and are more flexible in their planning. Accordingly, they are more likely to notice, and act on, the most credible and attractive marketing messages... However, DMOs and hotels can use some of the characteristics evident in this group to leverage tourists into desired activities. For instance, in this stage, tourists have often found 'new best friends' at the destination, and group activities involving the tourists and friends are more likely to be considered in a favorable light (Anantamongkolkul et al. 2017).

En estos ejemplos, el uso de la fórmula con be likely to se emplea con el efecto atenuador descrito anteriormente. Lo que se persigue con esto es evitar la imposición del punto de vista de los autores, por lo que sería un mecanismo de cortesía negativa, siguiendo el modelo de Brown y Yule (1987).

\subsection{Conclusión}

En este capítulo, se ha llevado a cabo una exposición de aquellos aspectos lingüísticos que guiarán el análisis de los modales que se presentan en el capítulo cuarto de este trabajo. Para ello, se ha revisado y precisado la noción de modalidad que se sigue en este 
estudio y que, como he apuntado más arriba, tiene como finalidad la evaluación de la proposición modulada, por lo que se trataría de un mecanismo de perspectivización, como se ha señalado en la literatura científica (cf. Papafragou 1998; Closs Traugott 2003; Efstathiadi 2010; Kirkham 2011). Esta evaluación puede tener diferentes efectos comunicativos y varía según el contexto y los propios significados de los verbos modales empleados. Se ha descrito también los conceptos de objetividad, subjetividad e intersubjetividad, que me permitirán explicar algunos usos de los verbos modales identificados en las introducciones y las conclusiones de los artículos científicos en la disciplina de turismo.

Además se ha realizado una descripción del artículo científico en turismo como género textual. Por este motivo, se describe la estructura del artículo de investigación en turismo siguiendo el modelo teórico de la lingüística sistémico-funcional, por la que esta configuración se indica en términos de secciones. El patrón organizativo del género en turismo ha sido descrito en Alonso-Almeida y Álvarez-Gil (2021a), que constituye el primer acercamiento al artículo de investigación en esta especialidad. En su trabajo, estos autores muestran la macroestructura del género mediante una muestra de artículos que fueron segmentados cada uno de manera autónoma para identificar correctamente las secciones. Siguiendo este estudio, se evidencian siete secciones, de las que solo la introducción aparece en todos los artículos analizados. El resto de las secciones no siempre aparecen como tales en las muestras seleccionadas, tal y como manifiesta en el potencial genérico ofrecido por estos autores.

Una parte fundamental es la caracterización de las secciones en cuanto a sus funciones y a la lengua usada y que será de mucha utilidad en la identificación de los textos que se ha llevado a cabo para el corpus, como se verá en el próximo capítulo. Si bien todas las 
secciones parecen tener funciones específicas, la discusión y la conclusión comparten alguna de ellas. Esto se debe probablemente a que algunos artículos no presentan estas dos secciones a la vez, por lo que las funciones de la discusión se asumen en la conclusión y al revés. 


\section{Metodología y corpus de análisis}

\subsection{Introducción}

Este capítulo presenta una descripción del corpus de trabajo y de la metodología seguida para la compilación textual y el análisis de la modalidad en este trabajo. Para garantizar que los textos que componen el corpus de introducciones y de conclusiones se han identificado correctamente, se lleva a cabo un análisis de la lengua de todas las secciones de los artículos de investigación en turismo seleccionados para la compilación. Con esto se justificarían todas las inclusiones de las secciones denominadas "introducción" y "conclusión" en el corpus, ya que todas responden a unos criterios funcionales y léxicogramaticales, aplicando los criterios que se recogen en Alonso-Almeida y Álvarez-Gil (2021), como se ha descrito en el capítulo anterior.

Dicho esto, la estructura de este capítulo es la siguiente. En la sección 3.2, se describe el método de trabajo y se presenta el corpus para el análisis de las perífrasis modales en la sección 3.3. En la sección 3.4, se muestran patrones específicos de lengua que se asocia con cada sección de la estructura del artículo de investigación con ejemplos concretos extraídos de los artículos completos de los que se han extraído las introducciones y las conclusiones que conforman el corpus de trabajo para el análisis de los verbos modales en el capítulo 4. 


\subsection{Metodología de análisis}

El análisis de las perífrasis modales en este trabajo se basa en las formas encontradas mediante análisis computerizado en un corpus de introducciones y conclusiones en artículos de investigación en turismo, como se ha explicado en la introducción. Estas secciones extraídas de los artículos de turismo para compilar nuestro corpus se han convertido en texto plano legible para su uso con las herramientas de CasualConc (Imao 2020) para el análisis computarizado de estos textos. El conjunto permite la búsqueda de formas modales según la cadena can/could/may/might/must/will/would/shall/should según cada una de las secciones de los artículos aquí considerados. El número de palabras del material introductorio de los artículos científicos es de 100.363, y otro conjunto de 83.156 palabras corresponde al material de conclusión de los artículos científicos. Para comparar los resultados, los datos se han normalizado a 10.000 palabras.

La identificación de las secciones de introducción y conclusión en los documentos seleccionados se ha llevado a cabo usando la información en Alonso-Almeida y ÁlvarezGil (2021a), además la mayoría de los textos incluían los nombres de las secciones, por lo que el grado de fiabilidad es alto. En aquellos casos en los que se haya usado el término "conclusion" juntamente con "discussion", nos hemos asegurado de que los contenidos que se incluyen sean exactamente los que corresponde a una conclusión. La consulta del corpus por sección aplicando las plantillas léxico-gramaticales descritas en la sección 3.4 ha devuelto los ejemplos que se incluyen a continuación, justificando así la división de las secciones realizada.

En cuanto al método, para obtener los datos de las perífrasis modales, se ha introducido la cadena can/ could/may/ might/ must/ will/ would/shall/ should en CasualConc para 
obtener las concordancias con estas formas en las introducciones y en las conclusiones. Además, estas perífrasis se han clasificado según los significados modales encontrados en estos textos. Estos significados se han identificado siguiendo la descripción ofrecida en la sección del marco teórico en el segundo capítulo para las categorías de modalidad epistémica, deóntica y dinámica.

Como ya se ha mencionado, el contexto es fundamental para identificar los significados modales y la función pragmática que desempeñan los verbos en cada una de las secciones del artículo científico que se analizan en este estudio. En este sentido, von Fintel (2006: 22-23) sostiene que "modal expressions have in of themselves a rather skeletal meaning and it is only in combination with the background context that they take on a particular shade of meaning (such as epistemic or deontic)", aunque estos "are not entirely subject to the whims of context but impose their own preferences as to what kind of modal meaning they would like to express". Siguiendo estas ideas, en los análisis, se informa sobre la frecuencia de la aparición de las formas modales que aparecen en las introducciones y las conclusiones del artículo científico en turismo. Estas ocurrencias de perífrasis modales se presentan, primero, en su conjunto $y$, seguidamente, por cada forma detectada. Para determinar la existencia de variación entre las secciones y el efecto real de esta, se usará el análisis estadístico denominado t-test, que indica si dicha variación es significativa en dos grupos de datos. Con estos datos, se calcula la variable $d$ de Cohen, que indica tres tamaños de efecto, a saber (a) pequeño: $d \leq 0,2$, (b) medio: $d$ $\leq 0,5, y(c)$ grande: $d \leq 0,8$

Para el cálculo de la variación, en el caso de los significados modales, se usa la prueba estadística de log-likelihood (LL), cuyos valores para cada forma modal se pueden calcular la Log-likelihood and effect size calculator que se encuentra disponible de manera 
gratuita en http://ucrel.lancs.ac.uk/Ilwizard.html. Esta calculadora requiere de los datos de frecuencia no normalizada para cada sección y por perífrasis modal junto con el total de palabras que componen el corpus por sección. Esta prueba LL funciona bien para determinar el efecto de variación existente entre dos grupos de variables en corpus pequeños, como en el caso de nuestro corpus de introducciones y conclusiones de artículos científicos, que no supera las 200.000 palabras.

Este es, junto con el enfoque que se desarrolla en este capítulo sobre la estructura del artículo de investigación en turismo, el método usado para el análisis de las perífrasis modales en el corpus de introducciones y conclusiones en el capítulo 4. En definitiva, los análisis de los textos permiten los cálculos estadísticos necesarios para obtener unas conclusiones cuantitativas que sirven de fundamento para un análisis cualitativo de los resultados, como se verá en el siguiente capítulo.

\subsection{Metodología de compilación textual: Corpus de introducciones y conclusiones de $\mathrm{Al}$ en turismo}

Las introducciones y conclusiones que sirven de base para esta tesis doctoral parten de un conjunto de 160 artículos de investigación encontrados en revistas publicadas entre los años 2015 y 2018 con un puntaje superior a nueve puntos en el índice de visibilidad ICDS (Secondary Composite Index Broadcasting), que puede ser consultado en MIAR (http://miar.ub.edu). Un índice de este tipo indica que una revista con nueve puntos o más de ICDS está obligada a aparecer como incluida en varias bases de datos internacionales. La pertinencia de este criterio para la selección de artículos de revistas en nuestro corpus es que garantiza, en primer lugar, que la revista goce de un reconocimiento de prestigio y, en segundo lugar, que la revista tenga una distribución 
amplia en la comunidad científica a la que sus contenidos está destinada. En principio, se entiende las revistas con este nivel mínimo de clasificación ICDS poseen normas reconocidas de investigación que aseguran la calidad en la edición y en el contenido.

En este trabajo se han seleccionado las revistas Journal of Travel Research, Journal of Vacation Marketing, Tourism Economics y Tourist Studies para extraer los textos, pues cumplen con este criterio de visibilidad. Puesto que el trabajo persigue el análisis de las perífrasis modales en inglés, será esta la lengua de los textos que se compilen. Esto no significa que la autoría provenga de una persona nativa y, por lo tanto, con lengua inglesa como L1. Por lo general, aunque utilicen alguna variedad geográfica inglesa, se tiende tendencia a establecer como criterio la variante 'nativo' frente a 'no nativo'. Sin embargo, esta diferenciación suele estar justificada, casi siempre, por unos apellidos ante el desconocimiento real de la identidad de la persona que ostenta dicho apellido. Así, alguien cuyo apellido sea Smith se le supone una educación en inglés, incluso si esa persona tiene dicho apellido por descendencia casual de una persona de origen anglosajón, pero esta persona, sin embargo, se ha criado en un país cuya L1 no es inglés y su contacto exclusivo con esta lengua es como L2. Por otra parte, nadie puede afirmar que una persona cuyo apellido sea Fernández no se haya criado en el Australia y sea de segunda o tercera generación de inmigrantes, por lo que su L1 sería la lengua inglesa. Como se destaca en Tribble (2017: 34), un apellido no puede realmente indicar si el inglés es o no la L1 de un/a hablante. Añade que lo que "can identify, however, are shared features of lexis, grammar, and discourse convention, which clearly show that the texts are grounded in specialist academic written genres".

Incluso, en el caso de los hablantes no nativos, la mayoría de las revistas exigen que el artículo sea editado por un hablante L1 antes de su publicación, y estos artículos pueden 
dar lugar a una mezcla de idiolectos en los que no siempre es posible distinguir con seguridad la identidad. Por otra parte, la experiencia de la disciplina parece anular la distinción entre nativo y no nativo, como sugieren los resultados de Römer (2009: 99): "when we deal with advanced-level academic writing, we actually move beyond the native/non-native distinction and that, in this context, experience or expertise is a more important aspect to consider than nativeness". Así, Mauranen $(2018: 113,116)$ dice que, en realidad, la noción de idiolecto es más adecuada y fiable en las descripciones de los textos. Tras lo expuesto, por lo tanto, hemos preferido analizar el idioma sin dar por sentado el origen de los/las hablantes.

Dicho esto, la composición de nuestro corpus de introducciones y conclusiones quedaría de la siguiente manera, como se ve en la tabla 1:

\begin{tabular}{|c|c|c|c|c|}
\hline Año & $\begin{array}{c}\text { Número de } \\
\text { artículos }\end{array}$ & Revistas & Sección & $\begin{array}{c}\text { Número de } \\
\text { palabras }\end{array}$ \\
\hline 2015 & 15 & Journal of Travel Research & Introducción & 100.363 \\
\hline 2016 & 58 & Journal of Vacation & & \\
\hline 2017 & 37 & Marketing Tourism & & \\
\hline 2018 & 50 & $\begin{array}{l}\text { Economics } \\
\text { Tourist Studies }\end{array}$ & Conclusión & 83.156 \\
\hline
\end{tabular}

Tabla 1. Corpus.

Como es lógico, la extracción de secciones específicas de un artículo de investigación en turismo requiere primero de una caracterización de este género que permita, de manera fiable, la identificación por razones funcionales y lingüísticas de dichas secciones. Por este motivo, siguiendo lo expuesto sobre la caracterización del artículo científico en turismo en el contexto de la teoría sistémico-funcional en el capítulo 2, se lleva a cabo un reconocimiento de las secciones contenidas en los artículos de investigación seleccionados para extraer las introducciones y las conclusiones. Los datos bibliográficos 
de estos textos se incluyen en el apéndice de esta tesis. La referencia a los textos del corpus (introducción y conclusión) se realiza siguiendo el formato apellidos de los autores seguido del año de publicación e irán precedidos de un número entre paréntesis. En el caso de los ejemplos referidos al resto de las secciones del artículo de investigación (enfoque, diseño, análisis, discusión), pero que no forman parte del corpus de trabajo, se referencian, además, incluyendo la página del fragmento.

\subsection{Resumen}

En este capítulo, se ha presentado la metodología empleada para la construcción del corpus de trabajo y para el análisis de las perífrasis modales en un corpus de introducciones y conclusiones. Para identificar las secciones que forman parte de nuestro corpus (introducción y conclusión), se ha analizado el uso de la lengua de estas secciones, pues existen una serie de expresiones léxico-gramaticales que aparecen con frecuencia y señalan las funciones de cada sección, lo que justifica su empleo a la vez que ayuda a distinguir una sección de otra. En este capítulo, se ofrecen ejemplos de estas expresiones de aquellos artículos de investigación de turismo que han servido de base para construir el corpus de introducciones y conclusión. Con esto, se verifica la existencia de estos patrones en el artículo científico en turismo y, sobre todo, esto permite garantizar que los textos incluidos en el corpus de trabajo son realmente introducciones y conclusiones. Además, y sin ser un objetivo de este capítulo, se ha evidenciado que algunas de estas estructuras se utilizan como mecanismos que manifiestan el punto de vista del hablante. Como se ha mostrado aquí, estos mecanismos aparecen sobre todo en las secciones denominadas discusión y conclusión, pues se presentan ahí el resultado cuantitativo y, especialmente, cualitativo de los análisis de los datos examinados. 
En el siguiente capítulo, se lleva a cabo el estudio de los verbos modales en las introducciones y las conclusiones de los artículos de turismo, toda vez que han quedado identificadas estas partes en los textos seleccionados en este capítulo para asegurar la inclusión exclusivamente de dichas secciones. En el caso de que aquel artículo que presenta conclusión no forma parte del corpus de trabajo para el análisis de las perífrasis modales. Como se verá, estas perífrasis también se usan para expresar la perspectiva de los autores sobre la información que aportan usando el marco explicado en el Capítulo 2. 


\section{Resultados y discusión. La modalidad en introducciones y conclusiones en Al}

\subsection{Introducción}

En este capítulo, se analizan las perífrasis modales en las introducciones y las conclusiones extraídas de artículos científicos en turismo. En la sección 4.2, contiene los resultados obtenidos del análisis de los textos siguiendo la descripción del método de estudio con indicación de las herramientas y métodos estadísticos usados que se incluye en el capítulo 3. En la sección 4.3, se realiza una discusión de estos datos organizados por significado. Finalmente, se incluyen las conclusiones de este estudio en la sección 4.5.

\subsection{Análisis del corpus: las formas de las perífrasis modales}

El análisis del corpus indica el uso de las perífrasis modales can, could, may, must, may, might, will, would, shall y should con el número real de casos (NR) y con la frecuencia relativa (FR) por cada 10.000 palabras de todas estas formas modales que se detalla en la siguiente tabla: 


\begin{tabular}{lrrrr}
\hline Perifrasis modales & \multicolumn{3}{c}{ INTRODUCCIÓN } & CONCLUSIÓN \\
\hline & FR & NR & FR & NR \\
can & 24,51 & 246 & 34,39 & 286 \\
could & 3,69 & 37 & 19,36 & 161 \\
may & 10,06 & 101 & 31,87 & 265 \\
might & 2,29 & 23 & 4,93 & 41 \\
must & 1,00 & 10 & 3,37 & 28 \\
will & 11,36 & 114 & 15,75 & 131 \\
would & 5,88 & 59 & 12,87 & 107 \\
shall & 0,40 & 4 & 0,24 & 2 \\
should & 3,59 & 36 & 18,52 & 154 \\
Total & 62,77 & 630 & 141,30 & 1175 \\
\hline
\end{tabular}

Tabla 2. FR de las perifrasis modales en introducciones y conclusiones.

Como se deduce en esta tabla, son 1.805 las perífrasis modales que se han identificado en el corpus. El número de estas formas modales es mayor en la conclusión que en la introducción llegando a doblar el número de casos tanto en los casos reales como en las frecuencias relativas. La distribución de cada perífrasis en las dos secciones es muy desigual, lo que se aprecia de manera visual en el gráfico 2.

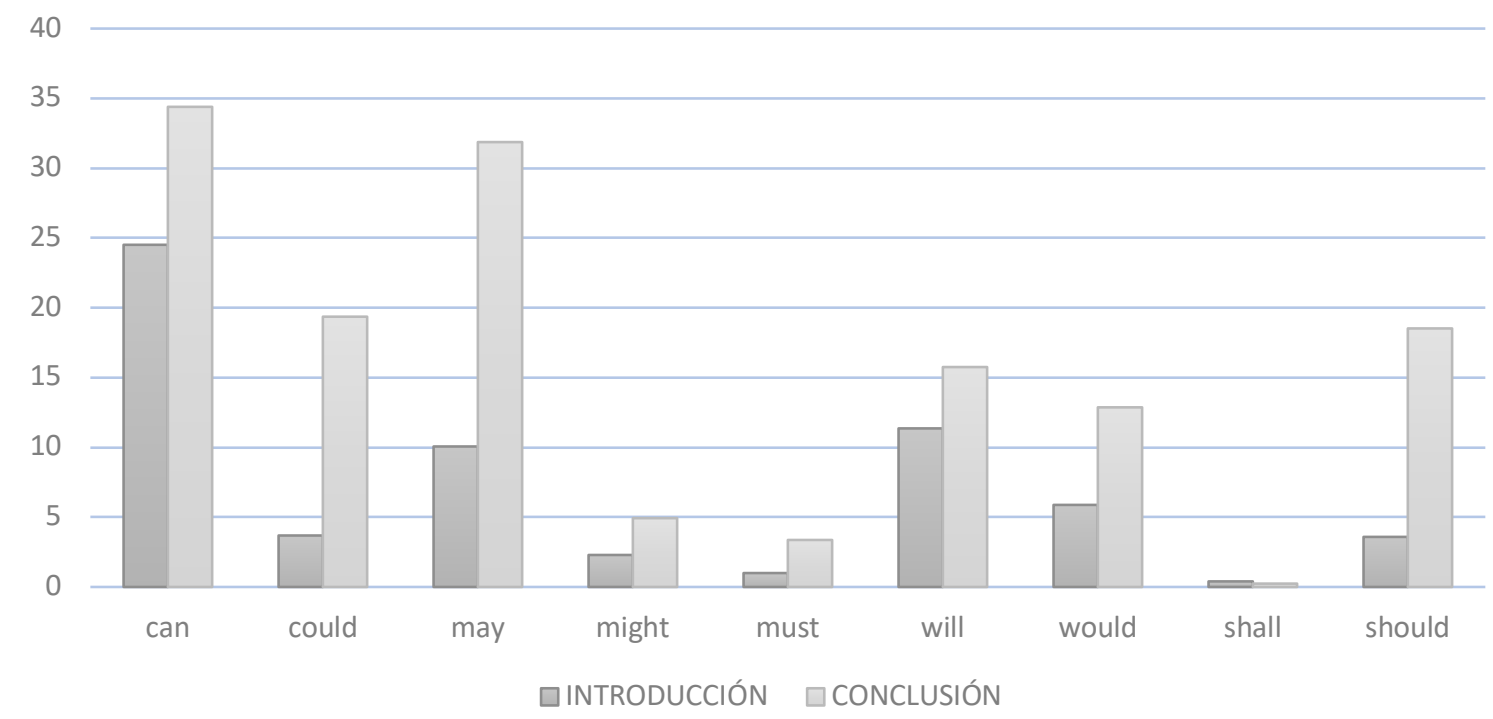

Gráfico 2. Distribución de perífrasis modales (FR 10,000 palabras). 
En este gráfico, se aprecia que las formas can, may, could, should, will y would son las más frecuentes en las introducciones por este orden. En el caso de las conclusiones, son can, will, may y would. En las dos secciones, can es la perífrasis más usada con mayor frecuencia en las conclusiones, si bien las formas que parecen marcar una diferencia más acentuada son may, should y could que registran una mayor presencia en las conclusiones.

En la tabla 3 se incluyen los datos relativos al t-test y la variable del tamaño de efecto Cohen $d$ :

\begin{tabular}{lcrrr}
\hline Perifrasis & t-test & $p$ & $d f$ & Cohend \\
\hline Todas las perifrasis & $-8,93$ & 0,001 & 211,73 & $-1,109$ \\
\hline
\end{tabular}

Tabla 3. Todas las perifrasis: T-test y Cohen d.

El análisis paramétrico mediante una prueba $t=-8,93$, con un índice de probabilidad de $p=0,01$ y un grado de libertad de 211,73 , indica que existe diferencia entre el uso de estas perífrasis en las dos secciones. La variable $d$ de Cohen es de $-1,109$, lo que indica un efecto grande en términos generales. Se constata con esta prueba las conclusiones presentan mayor variación en cuanto al número de perífrasis modales en sus frecuencias relativas, excepto en el caso de shall, que ocurre con mayor frecuencia en las introducciones que en las conclusiones. A continuación, se determinará si estas variaciones son significativas entre las dos secciones con los mismos cálculos estadísticos realizados para cada forma.

Como se indica en la tabla 2, arriba, can es, con diferencia la forma más usada en el corpus con un FR de 34,39 en las conclusiones y de 24,51 en el caso de las introducciones. Los 
valores mínimos y máximos por texto en nuestro corpus de esta forma se presenta visualmente en el gráfico 3.

$\circ$

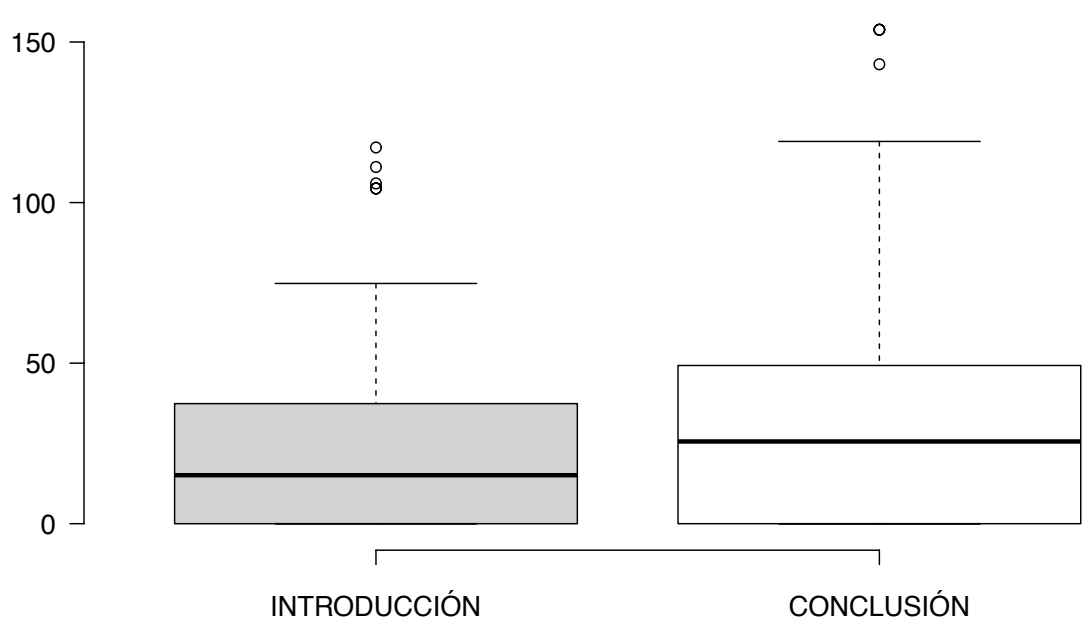

Gráfico 3. Boxplot can (FR 10,000).

En este gráfico, se aprecia que, si bien, las introducciones presentan valores máximos de can la media es mayor en el caso de las conclusiones. La tabla 4 muestra las estadísticas relativas a la variación de uso de can en la sección introducción y la sección conclusión.

\begin{tabular}{lrrrr}
\hline Perifrasis & t-test & $p$ & $d f$ & Cohen d \\
\hline can & $-2,35$ & 0,0183 & 251.07 & -0.29
\end{tabular}

Tabla 4. Perifrasis can: T-test y Cohen d. 
El valor $t=-2,35$, con un índice de probabilidad de 0,0183 y un grado de libertad de 251,07, refleja que realmente hay variación, si bien el efecto de esta es medio, como muestra el valor Cohen $d=-0,29$.

Algunos ejemplos de can encontrados en el corpus son los siguientes:

(1) (Introducción) In this view, things exist as meaningful entities independent from consciousness and experience that they have truth and meaning residing in them as objects and that careful research can reach objective truth and meaning (Crotty, 1998). Since variables and their relationships can be identified and measured, the authors of the current study approach the topic of interest with positivism as a theoretical perspective (Atadil et al. 2017).

(2) (Conclusión) This can be principally explained by the presence of China and its hotels in the sample. However, when technical-economic efficiency measures calculated through DEA are incorporated, no mediating effect of this variable can be observed. Therefore, with the available data, we can say that efficiency acts more as a covariate than a mediator (Mendieta-Peñalver et al. 2018).

Estos ejemplos muestran el uso de la perífrasis can en las dos secciones con un marcado significado dinámico en todos los casos. Esta forma se repite en dos oraciones consecutivas en las dos muestras. Como se verá en la última sección 4.4, además de indicar perspectiva, las repeticiones de las formas modales en un párrafo contribuyen a la organización del contenido del texto argumentativo. 
La perífrasis could también presenta más casos en las conclusiones que en las introducciones, como se muestra en las FR de la tabla 2 y visualmente en el gráfico 4.

\section{COULD}

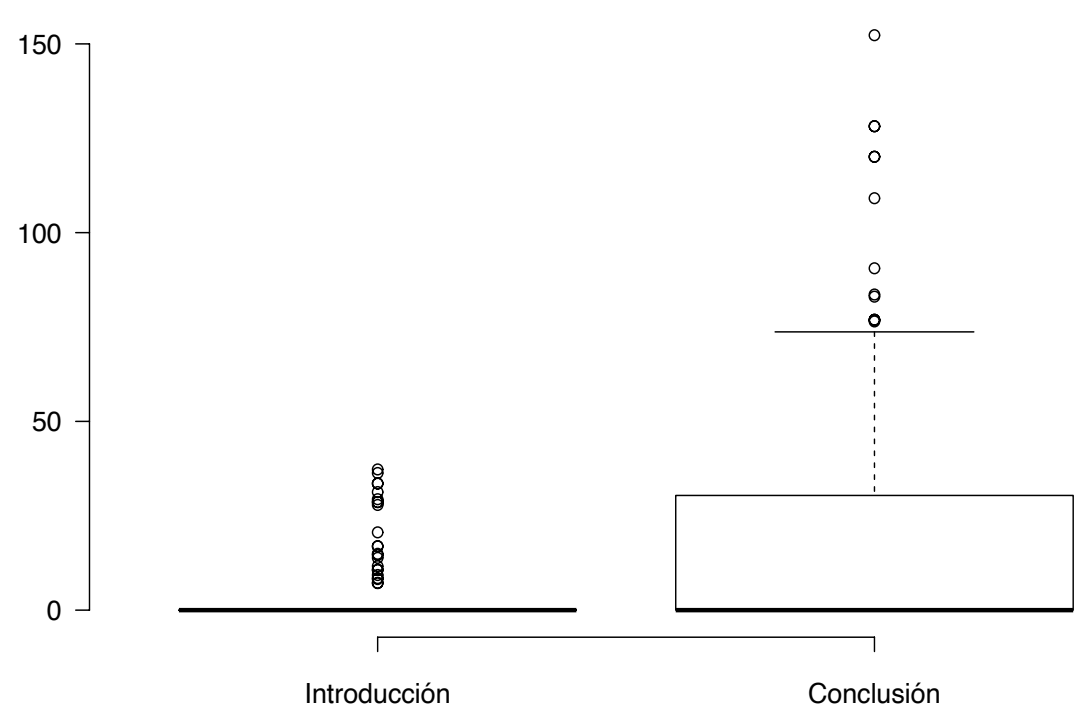

Gráfico 4. Boxplot could (RF 10,000).

En este gráfico, se aprecia con claridad que la conclusión tiene textos con mayor índice de presencia de could que la introducción. La media de textos con mayor frecuencia de esta forma es también mayor en el caso de la conclusión. Esta variación se recoge en los datos estadísticos para esta perífrasis en el corpus en la tabla 5:

\begin{tabular}{lrrrr}
\hline Perifrasis & t-test & $p$ & $d f$ & Cohen $d$ \\
\hline could & -5.8 & 0,001 & 144,24 & $-0,73$
\end{tabular}

Tabla 5. Perifrasis could: T-test y Cohen d. 
Se constata en la tabla 5 que la variación es realmente significativa con un valor $t=-5,8$, un índice de probabilidad de 0,001 y un grado de libertad de 144,24. Como muestra el valor Cohen $\mathrm{d}=-0,73$, el efecto es medio.

Los siguientes fragmentos muestran formas de esta perífrasis en el corpus, por sección:

(3) (Introducción) Moreover, as emphasized by the industry, if the replacement eventuates, affecting only against Chinese visitors, this could easily be seen as a form of market discrimination, perhaps with political implications (Pham et al. 2018).

(4) (Conclusión) For example, future studies could compare guides on the DWEWT with other examples of official and unofficial media tourism within a specific geographical area or across different regional, national and international contexts. Alternatively, further research could analyse how the status afforded to different forms of (guided) tourism are positioned within the tourism field to better understand the variety of positions available and their relationship to systems of cultural power (Garner 2017).

Estos ejemplos muestran el empleo de could en el corpus. En los dos casos, el uso es epistémico con la indicación de la probabilidad de que las acciones moduladas por esta perífrasis puedan ocurrir. La función, como se verá en la sección 4.4, es diferente en las dos secciones y el sentido de recomendación para que se lleven a cabo una serie de actividades por parte de investigadores es patente en la conclusión en el último ejemplo. 
La perífrasis may aparece con mayor frecuencia relativa, en concreto de 10,06 , en las conclusiones con respecto a las introducciones que presentan un valor de 31,87, como se ha mostrado en la tabla 2. A continuación en el gráfico 5 se presentan los valores máximos y mínimos:

\section{MAY}

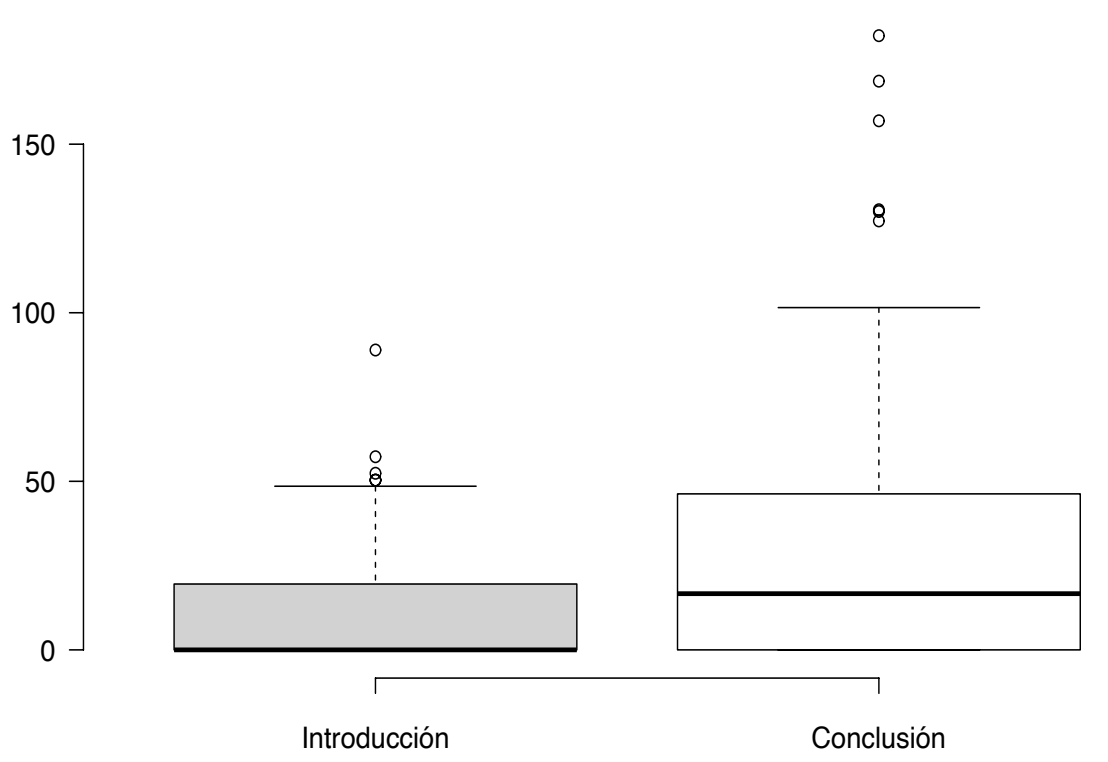

Gráfico 5. Boxplot may (FR 10,000).

El gráfico refleja visualmente que el subcorpus de conclusiones contiene más textos con la perífrasis may que en el caso del subcorpus de introducciones. La media también es mayor en las conclusiones. Los valores estadísticos incluidos en la tabla 6 confirman esta variación: 


\begin{tabular}{lrrrr}
\hline Perifrasis & t-test & $p$ & $d f$ & Cohen $d$ \\
\hline may & $-4,96$ & 0,001 & 170,93 & $-0,62$ \\
\hline
\end{tabular}

Tabla 6. Perifrasis may: T-test y Cohen d.

La prueba estadística $t$ da como resultado $-4,96$ y una probabilidad $p=0,001$ y un grado de libertad de 170,93. El valor $d$ de Cohen es -0,62 confirma, por lo tanto, que el efecto de variación en cuanto al uso de may es grande entre las secciones.

Los siguientes ejemplos contienen formas de may:

(5) (Introducción) Here, however, I want to discuss the project's framing as a heritage destination, and how such a positioning may contribute to its legitimacy, particularly as it relates to tourists' experience of the site (Jethro 2016).

(6) (Conclusión) Second, snowball sampling is a nonprobability approach that may lead to sampling bias, hence limiting the general applicability of the findings. These limitations, however, do not diminish the significant contributions this study makes to the research literature. A future study may provide a longitudinal overview of the development of IPO strategies in the travel agency industry (Huang y Chang 2018).

Como se discutirá en la sección 4.3, may se usa especialmente para indicar posibilidad y probabilidad, aunque también presenta valores dinámicos para referirse a la capacidad que tienen las personas o las cosas para llevar a cabo una acción. En estos ejemplos, su empleo es para indicar probabilidad epistémica en todos los casos de esta forma. Como 
se aprecia en el caso de la conclusión, al igual que en can y could, la perífrasis may presenta consejos de acción investigadora en el futuro.

En el caso de la perífrasis might, como se indica en la tabla 2, esta forma muestra variación en su uso en las introducciones $(2,29)$ y las conclusiones $(4,93)$, con los valores máximos y mínimos que se presentan visualmente en el gráfico 6:

\section{MIGHT}

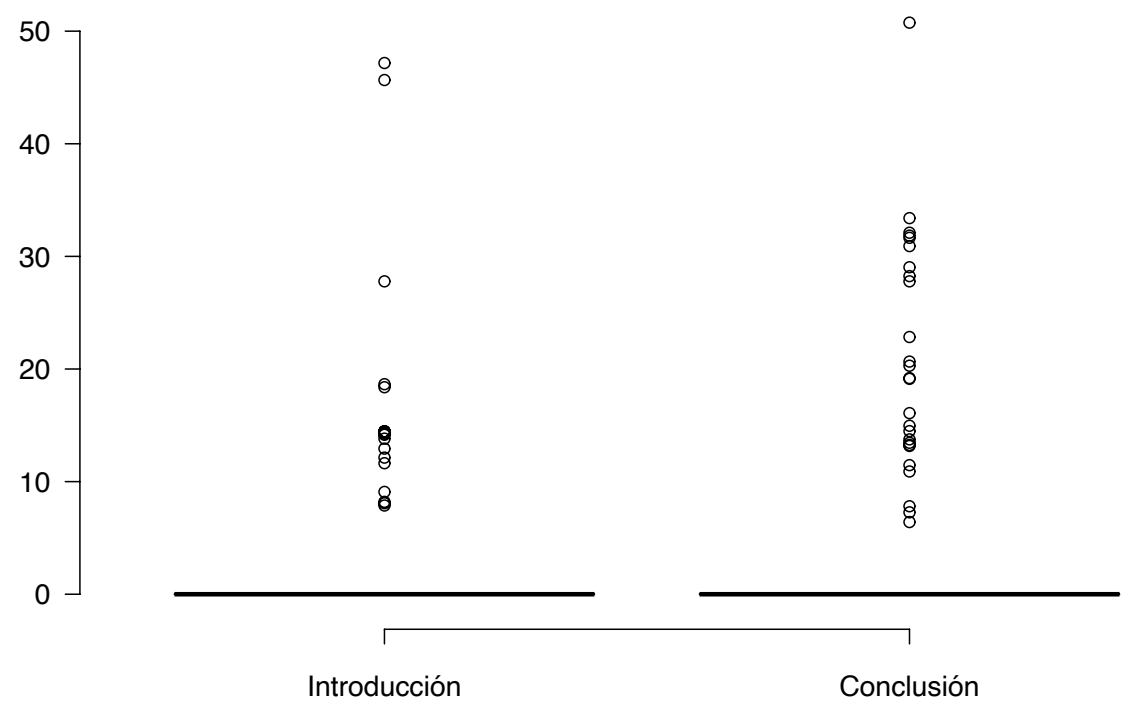

Gráfico 6. Boxplot might (FR 10,000).

Este gráfico revela claramente la similitud en cuanto a los niveles máximos, mínimos y medios en el uso de might para cada sección que componen el corpus. Los datos de variación se incluyen en la tabla 7: 


\begin{tabular}{lrrrr}
\hline Perifrasis & t-test & $p$ & $d f$ & Cohen $d$ \\
\hline might & $-1,68$ & 0,094 & 237,14 & $-0,21$
\end{tabular}

Tabla 7. Perifrasis might: T-test y Cohen d.

Como se aprecia en esta tabla, la variable de Cohen $d=-0,21$ señala un efecto pequeño en cuanto a la significación de la variación en el uso de might entre secciones. El valor estadístico de $t$ es $-1,68$, con una probabilidad de 0,094 y un grado de libertad 237,14.

Estos ejemplos ilustran su uso en las introducciones y en las conclusiones:

(7) (Introducción) The term stopover might indicate a temporary sense of placelessness, akin to the liminality apparent in Augé's (1995) notion of non- places (Lund et al. 2017).

(8) (Conclusión) This study was intentionally focussed on ways in which musicians tailor musical activities to the opportunities and imperatives associated with a tourist-orientated environment; so future research might aim to supplement this focus on musical 'producers' with a more detailed examination of the interactions between musicians and tourists in relation to the dynamics of musical events... Future research might also provide a more detailed examination of connections between musicalcultural activity and environmental activism on Fernando de Noronha and explore the implications for cultural-environmental activism within other tourist locations (Fitzgerald y Reis 2016). 
En estos ejemplos, todos los casos de might indican probabilidad tanto en la introducción como en las conclusión. En esta última sección, la perífrasis modal se repite en dos ocasiones y se emplea para proponer nuevamente sugerencias para la investigación evitando la imposición por parte de los autores, como se explicará con detalle en la sección dedicada las funciones de las perífrasis modales.

En el caso de la forma modal must, esta forma es más frecuente en las conclusiones con una frecuencia relativa de 3,37 frente a las introducciones que tienen una frecuencia de 1, como se indica en la tabla 2. Sin embargo, presenta los valores más altos en textos que corresponden a las introducciones, lo que se aprecia visualmente en el gráfico 7.

\section{MUST}

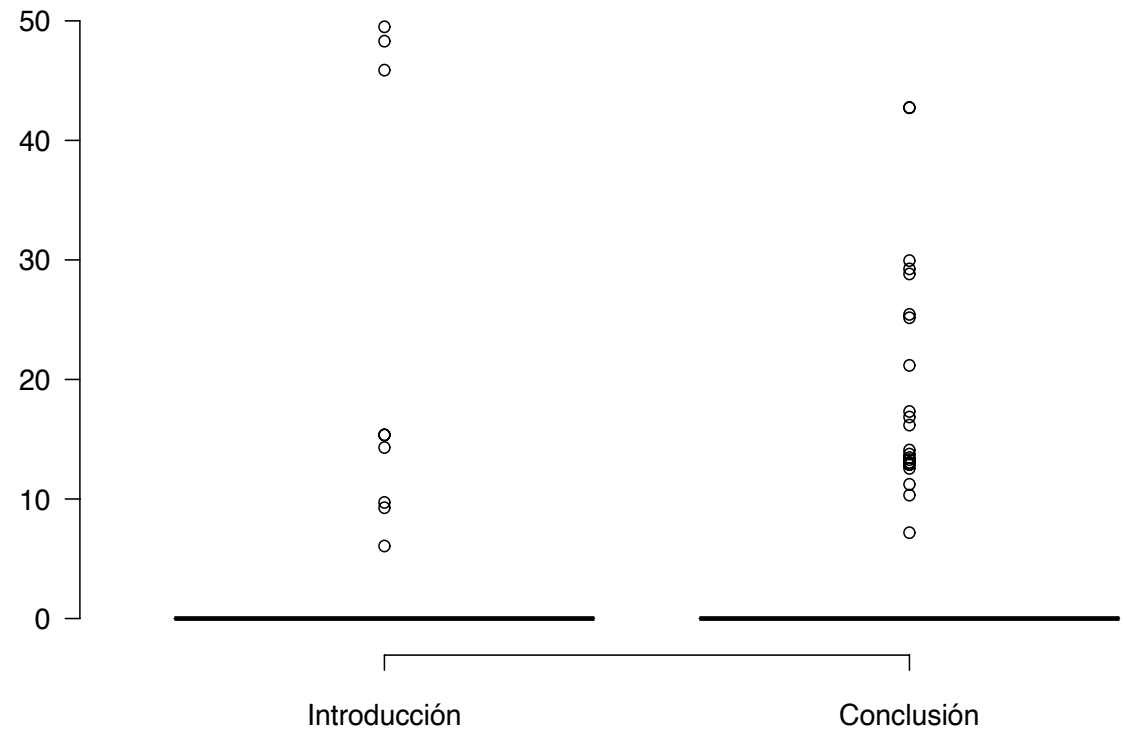

Gráfico 7. Boxplot must (FR 10,000). 
En este gráfico, los valores máximos se observan, como ya se ha apuntado, en los textos correspondientes a las introducciones, si bien también presenta los textos con los valores mínimos, aunque la diferencia es pequeña. Los valores estadísticos para estimar la existencia de variación se ofrecen en la tabla 8.

\begin{tabular}{lrrrr}
\hline Perifrasis & t-test & $p$ & $d f$ & Cohen d \\
\hline must & $-1,89$ & 0,06 & 252,65 & $-0,23$
\end{tabular}

Tabla 8. Perifrasis must: T-test y Cohen d.

De acuerdo con los datos de esta tabla, es evidente que must muestra variación significativa, si bien se trata de un efecto pequeño, esto es $d=-0,23$. El valor $t$ es 1,89 , como se indica aquí, con una probabilidad de $p=0,06$ y un grado de libertad de 232,65.

Los siguientes ejemplos presentan esta perífrasis en las dos secciones:

(9) (Introducción) As Hannam (2009) and others have pointed out, tourism must be seen as 'integral to wider processes of economic and political development' (p. 106; see also Edensor, 2007; Franklin, 2003; Sheller and Urry, 2006), and the use of a mobility lens offers much promise in this regard (Torabian y Mair 2017).

(10) (Conclusión) We do not suggest that heterosexuals cannot study homosexual people, but rather that in these scenarios, particular measures must be taken to establish trust, and to build a rapport with gay participants in 'oases' such as this gay resort (Vorobjovas-Pinta y Robards 2017). 
En los dos ejemplos que ilustran el uso de must en el corpus se nota el valor de esta forma para indicar necesidad. Si bien en el caso de la introducción, se trata de una cuestión metodológica en cuanto a la percepción del concepto de turismo en la investigación académica, en el caso de la conclusión se manifiesta la necesidad, según la perspectiva de los autores, de implementar acciones específicas en materia de lo que se denomina turismo para gays.

La forma will aparece en las dos secciones, como se aprecia en la tabla 2, arriba, con valores de 11,36 en las introducciones y de 15,75 en las conclusiones. La distribución entre los textos se percibe en el gráfico 8:

WILL

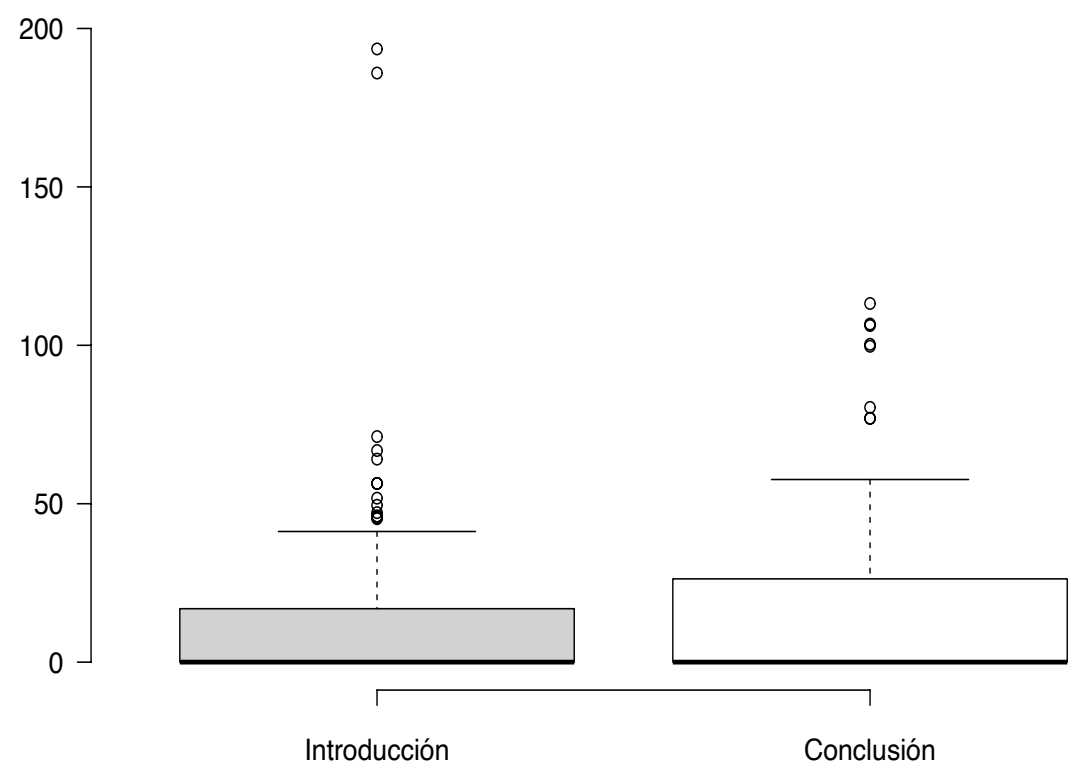

Gráfico 8. Boxplot will (FR 10,000). 
El gráfico 8 muestra, por otra parte, que las introducciones muestran los textos con las máximas frecuencias relativas, aunque la media es mayor en las conclusiones. En cuanto a los valores mínimos, existe coincidencia en los textos de las dos secciones. El grado de variación se deduce de los datos estadísticos que se incluyen en la tabla 9.

\begin{tabular}{lrrrr}
\hline Perifrasis & t-test & $p$ & $d f$ & Cohen $d$ \\
\hline will & $-0,73$ & 0,46 & 259,95 & $-0,09$
\end{tabular}

Tabla 9. Perifrasis will: T-test y Cohen d.

El análisis estadístico revela un valor de $t=-0,73$, con una probabilidad de 0,46 y un grado de libertad de 259,95. La variación es significativa con un valor obtenido para la variable de Cohen $d=-0,09$, lo que se interpreta como un efecto pequeño.

Los siguientes ejemplos incluyen esta forma:

(11) (Introducción) This article will continue with a review of relevant literature before proceeding to provide details of the data collection and methodology (Lee y Wilkins 2017).

(12) (Conclusión) This will raise a question as to whether or not the government is fully committed to the Potential target that both the government and the industry have set up... Consequently, this will lower business confidence in the sector, could affect the long-term investment strategy of investors and potentially be detrimental to trade and inflow of foreign direct investment (Neumayer, 2011; Song et al., 2012) (Pham et al. 2018). 
Las formas de will en estos ejemplos indican sentido de futuro que viene determinado por sus valores deónticos, si bien cada caso aporta un significado añadido. Esta perífrasis modal en la introducción indica la intención por parte de los autores sobre su posicionamiento teórico en el artículo. En el caso de los ejemplos de will en la conclusión, este indica consecuencia en el razonamiento que se plantea.

En cuanto a would, esta forma es más frecuente en las conclusiones con una frecuencia relativa de 12,87, mientras que la frecuencia es de 5,88 en las introducciones. El gráfico 9 muestra los valores medios, mínimos y máximos de would:

\section{WOULD}

○

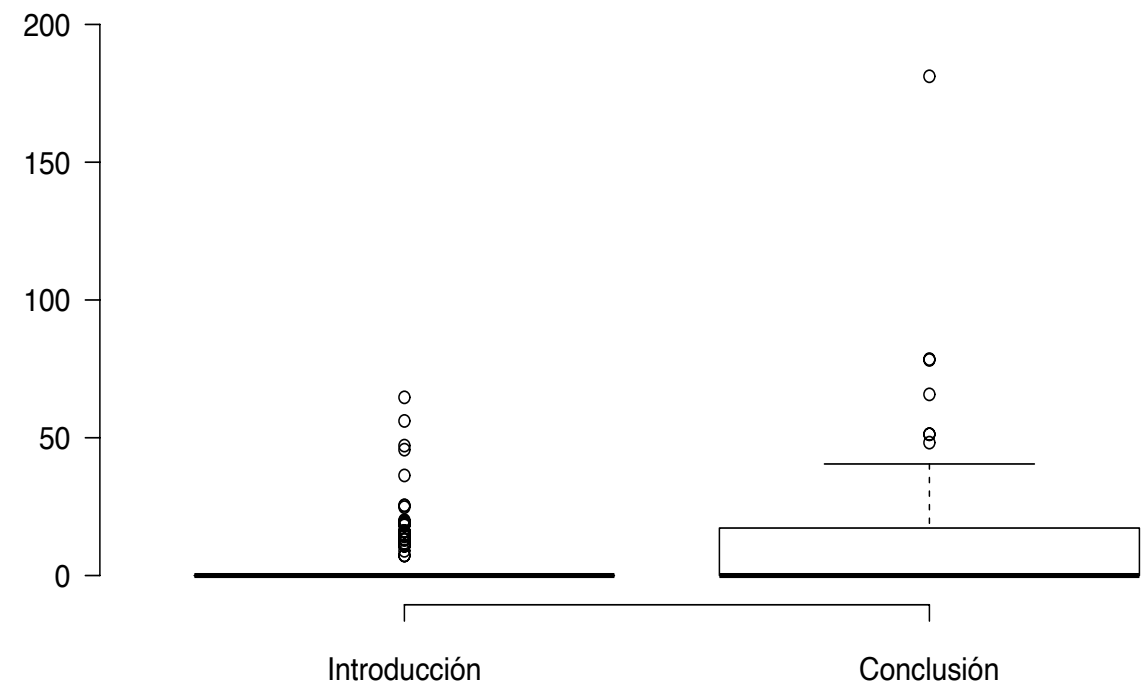

Gráfico 9. Boxplot would (FR 10,000). 
Las conclusiones muestran los niveles máximos y también la media mayor. Existen variación significativa entre el uso de esta forma en las secciones que se analizan en este trabajo, si bien el efecto es medio, como se aprecia en los datos estadístico en la tabla 10:

\begin{tabular}{lrrrr}
\hline Perifrasis & t-test & $p$ & $d f$ & Cohen d \\
\hline would & $-2,73$ & 0,007 & 150,99 & $-0,34$
\end{tabular}

Tabla 10. Perifrasis would: T-test y Cohen $d$.

Este efecto medio viene determinado por el valor de Cohen $d=-0,34$. El valor de $t$ es 2,73, con un índice de probabilidad de 0,007 y un grado de libertad de 150,99.

Los siguientes ejemplos incluyen casos de would en las introducciones y las conclusiones:

(13) (Introducción) The tourism industry in Australia has had good reasons to be concerned that the Federal government would continue to pursue revenue raising policies affecting the demand for inbound tourism, particularly as regards an expanding market such as China (Pham et al. 2018).

(14) (Conclusión) It would be a fallacious assumption that shopping is the core motive of travel to a shopping festival. An understanding of the motives would help in determining what drives a tourist towards a travel or activity. Tourists are likely to choose a destination that aligns with their motives. Research on travel motivation would enable the DMO in creating events, which would cater to the tourists' expressed motives for travel (Peter y Anandkumar 2016) 
La forma shall es con mucho la que presenta las menores frecuencias relativas con unos valores de 0,40 y 0,24, en las secciones introducción y conclusión, respectivamente, como se indica en la tabla 2, arriba. Los valores medios, mínimos y máximos se ofrecen en el gráfico 10.

\section{SHALL}

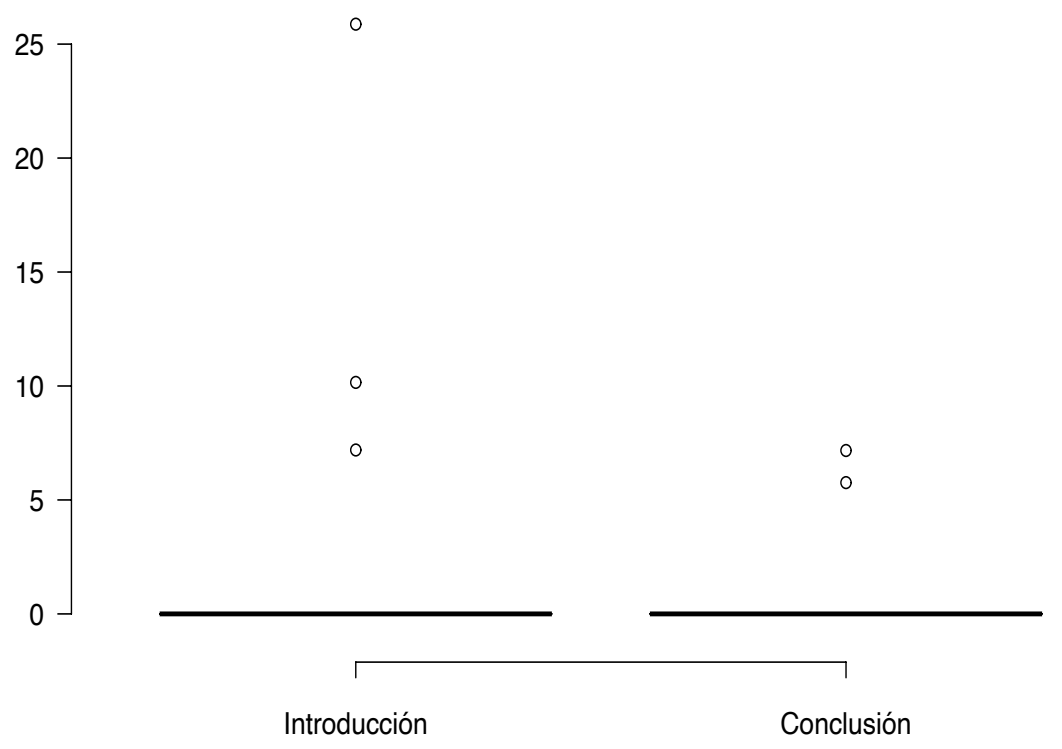

Gráfico 10. Boxplot shall (FR 10,000).

Este gráfico evidencia la escasa presencia de shall en el corpus. EL valor máximo se aprecia en los textos de las introducciones y el mínimo en los textos de las conclusiones. Los valores estadísticos para determinar si existe variación significativa se incluyen en la tabla 11. 


\begin{tabular}{lrrrr}
\hline Perifrasis & t-test & $p$ & $d f$ & Cohen d \\
\hline shall & 0,98 & 0,326 & 162,51 & 0,12 \\
\hline
\end{tabular}

Tabla 11. Perifrasis shall: T-test y Cohen d.

El análisis estadístico da como resultado un valor $t$ de 0,98, con una probabilidad de 0,326 y un grado de libertad de 162,51. El valor de Cohen $d$ es de 0,12 , lo que indica que, a pesar de existir variación en el uso de shall en las introducciones y en las conclusiones, esta es pequeña.

A continuación, se ofrecen algunos ejemplos de las introducciones y las conclusiones:

(15) (Introducción) In this article, we shall examine what kind of a gateway Iceland represents by focusing on how it is promoted as a tourism destination aimed at stopover visitors, continuously in play (Sheller and Urry, 2004), nevertheless designed and ordered to act as a gateway to 'elsewhere' (Coleman and Crang, 2002: 4; cf. Shields, 1989) (Lund et al. 2017).

(16) (Conclusión) I shall not make another inventory of the resemblances and differences between them for the Romanian case, among other things because the state of scientific research on this subject in Romania is almost non-existent (Banica 2016).

Estos ejemplos muestran casos de shall en las secciones del corpus. En los dos casos de shall, esta forma presenta un valor deóntico para expresar el deseo y la intención de los autores sobre acciones concretas. En la introducción, se indica la voluntad de los autores de llevar a cabo un un análisis específico sobre la promoción del turismo en Islandia. En 
la conclusión, shall introduce el compromiso de los autores para no realizar una nueva clasificación sobre el tema que se trata en relación al turismo en Rumanía, lo que se justifica posteriormente mediante la información la oración causal que introduce la partícula because.

Finalmente, should presenta un uso más marcado en las conclusiones con un valor de 18,52 que en las introducciones con un valor de 3,59, como se recoge en la tabla 2, arriba. Esta forma presenta los valores medios, máximos y mínimos que se muestran en el gráfico 11.

\section{SHOULD}

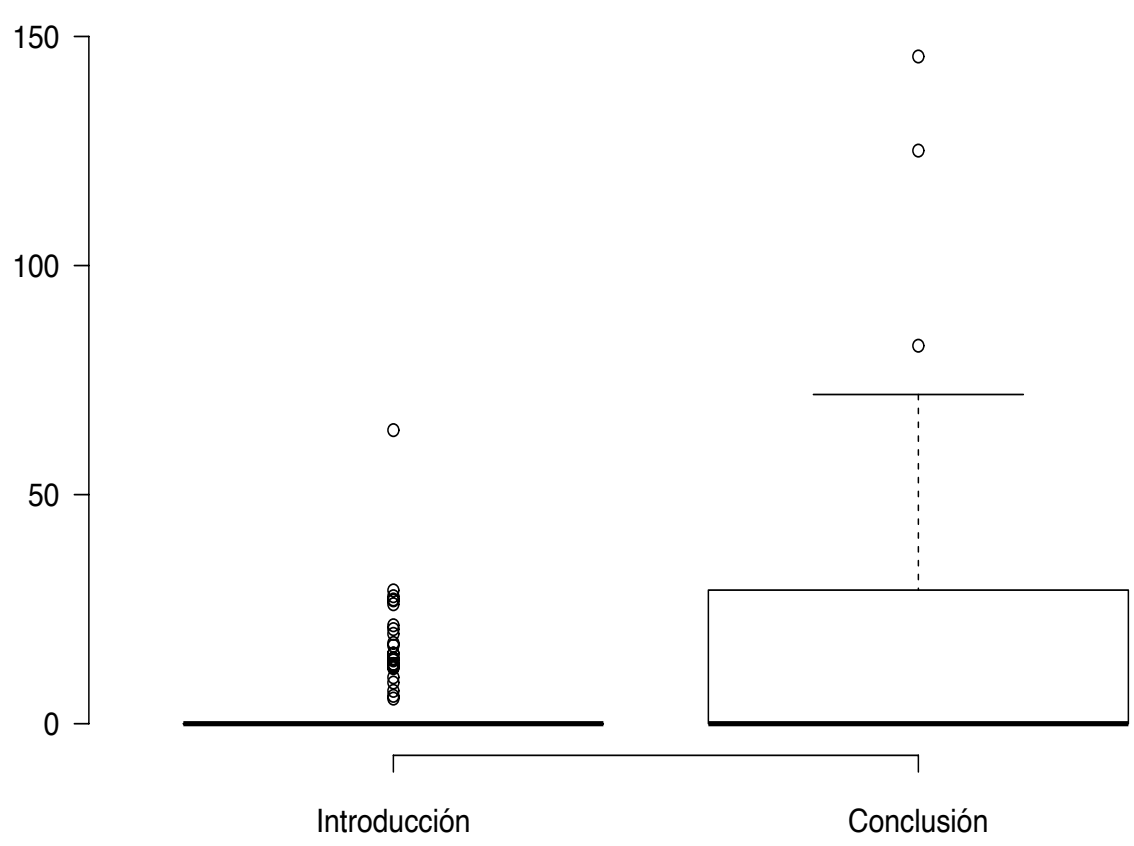

Gráfico 11. Boxplot should (FR 10,000). 
Los valores máximos se encuentran en el subcorpus de las conclusiones, al igual que el valor medio. Los valores estadísticos para determinar si existe variación significativa se ofrecen en la tabla 12.

\begin{tabular}{lrrrr}
\hline Perifrasis & t-test & $p$ & $d f$ & Cohen $d$ \\
\hline should & $-5,59$ & 0,001 & 156,92 & $-0,7$
\end{tabular}

Tabla 12. Perifrasis should: T-test y Cohen d.

La prueba estadística $t$ con un valor de $-5,59$, una probabilidad de $p=0,001$ y un grado de libertad de 156,92 revela variación en el uso de should en las dos secciones. El valor Cohen $d=-0,7$ revela que el tamaño del efecto de la variación es ciertamente grande.

Los siguientes ejemplos incluyen casos de should en la introducción y en la conclusión:

(17) (Introducción) Furthermore, Cohen and Cohen (2012) and Hannam (2008) suggested that tourism research within the contemporary mobilities paradigm should consider embodied and sensual practices of everyday from an interdisciplinary perspective (Bezzola y Lugosi 2018).

(18) (Conclusión) Aside from the need to further confirm these results through examining different cultures, some suggestions should be proposed to enhance the shopping for luxury items in HK, at least among Chinese tourists (Correia et al. 2018).

En estos dos ejemplos, se aprecia que should se usa para indicar recomendaciones. En la introducción, esta recomendación se refiere a aspectos metodológicos específicos en materia de investigación, mientras que la sugerencia en la conclusión está relacionada 
con beneficios concretos para la industria, lo que representa una función del uso de las perífrasis modales en los textos turísticos, como se describe en la sección 4.4, sobre las funciones de las perífrasis.

Alonso-Almeida y Carrió-Pastor (2017: 286-287) presentan un estudio exhaustivo de las formas verbales modales y sus significados en un corpus de artículos de investigación de lingüística y otro de artículos de investigación de ingeniería. En cuanto a la aparición de las perífrasis modales entre esos artículos y los de turismo, surgen algunas diferencias y similitudes. La forma can aparece con mayor frecuencia en la introducción en los artículos de lingüística e ingeniería, con frecuencias normalizadas de 40,72 y 36,33, respectivamente. Esto contrasta con la cifra de 24,51 en el corpus de textos de turismo. Sin embargo, existe mayor cercanía en la frecuencia de uso de can en las conclusiones con 34,39 y 30,45 en el corpus de turismo y lingüística respectivamente, aunque la diferencia es mayor en el caso del corpus de artículos de ingeniería, con una puntuación total de 40,42. En cuanto a could, esta forma es menos frecuente en las introducciones de textos de lingüística con 0,93. En cambio, su frecuencia relativa es de 3,69 en el corpus de turismo y de 2,20 en el de ingeniería. En el caso de las conclusiones, la forma could muestra una frecuencia normalizada de 19,36 en los trabajos de investigación sobre turismo y de 12,69 en los trabajos de investigación sobre lingüística. La diferencia está en el corpus de investigación de ingeniería, en el que no hay casos de could.

El modal may presenta menos casos en las introducciones de los trabajos de investigación en la disciplina de lingüística que en los otros corpus. En las conclusiones, no existe coincidencia entre ninguno de los corpus, y este modal tiene frecuencias normalizadas de 39,33 (lingüística), 31,87 (turismo) y 8,51 (ingeniería). El verbo might puede aparecer con más frecuencia en las introducciones de ingeniería y con menos frecuencia en las 
introducciones de turismo. La verdadera diferencia está en las conclusiones, con frecuencias normalizadas de 4,93 (turismo) y 22,84 (lingüística). Alonso-Almeida y CarrióPastor (2017) no identificaron casos de might en las conclusiones de ingeniería. El verbo must aparece en las introducciones de textos turísticos con una frecuencia relativa de 1 caso cada 10.000 palabras, con una presencia similar en las introducciones de textos de lingüística con 0,90. En el corpus de ingeniería, la frecuencia es mayor con 3,30. Esta forma está presente en las conclusiones de los textos en todos los corpus comparados y es más frecuente en los artículos de investigación de lingüística y turismo. La forma shall aparece en las introducciones y en las conclusiones de los artículos en turismo con un 0,40 y un 0,24 , respectivamente. No se detectaron casos en los otros corpus. En cuanto a should, aparece solo en las introducciones de turismo con una frecuencia de 3,59. En las conclusiones de los artículos en turismo, should tiene una frecuencia mayor, 18,52, que en los otros dos corpus: 14,89 (ingeniería) y 6,34 (lingüística).

Hay diferencia en la frecuencia de uso de will en todos los corpus comparados. Aparece con mayor frecuencia en los textos de lingüística con 27,76; de 13,21 y 11,36 en ingeniería y turismo, respectivamente. En las conclusiones, will aparece más a menudo en el corpus de textos de turismo con 15,75 y con una frecuencia menor en el corpus de ingeniería con 4,25 y en el corpus de lingüística con 3,81. Por último, would tiene frecuencias parecidas en las introducciones en los tres corpus mientras que la frecuencia es mayor en las conclusiones del corpus de lingüística con 25,37. En el corpus de turismo e ingeniería, muestra una frecuencia normalizada de 17,02 en ingeniería y 18,52 en turismo.

En conclusión, esta comparación con los datos obtenidos en el estudio de Alonso-Almeida y Carrió-Pastor (2017) revela que existe cierta variación de uso según la disciplina en lo 
que respecta a la forma de las perífrasis modales. Esto puede tenerse como indicio de que el significado modal que se encuentra en estas introducciones y conclusiones de los artículos de investigación en turismo en nuestro corpus es posible que también presente variación, como se analiza y describe en la siguiente sección.

\subsection{Los significados de las perífrasis modales}

El gráfico 12 presenta la distribución de los significados modales identificados en el corpus según la clasificación tripartita explicada en el capítulo 2, sección 2.3.

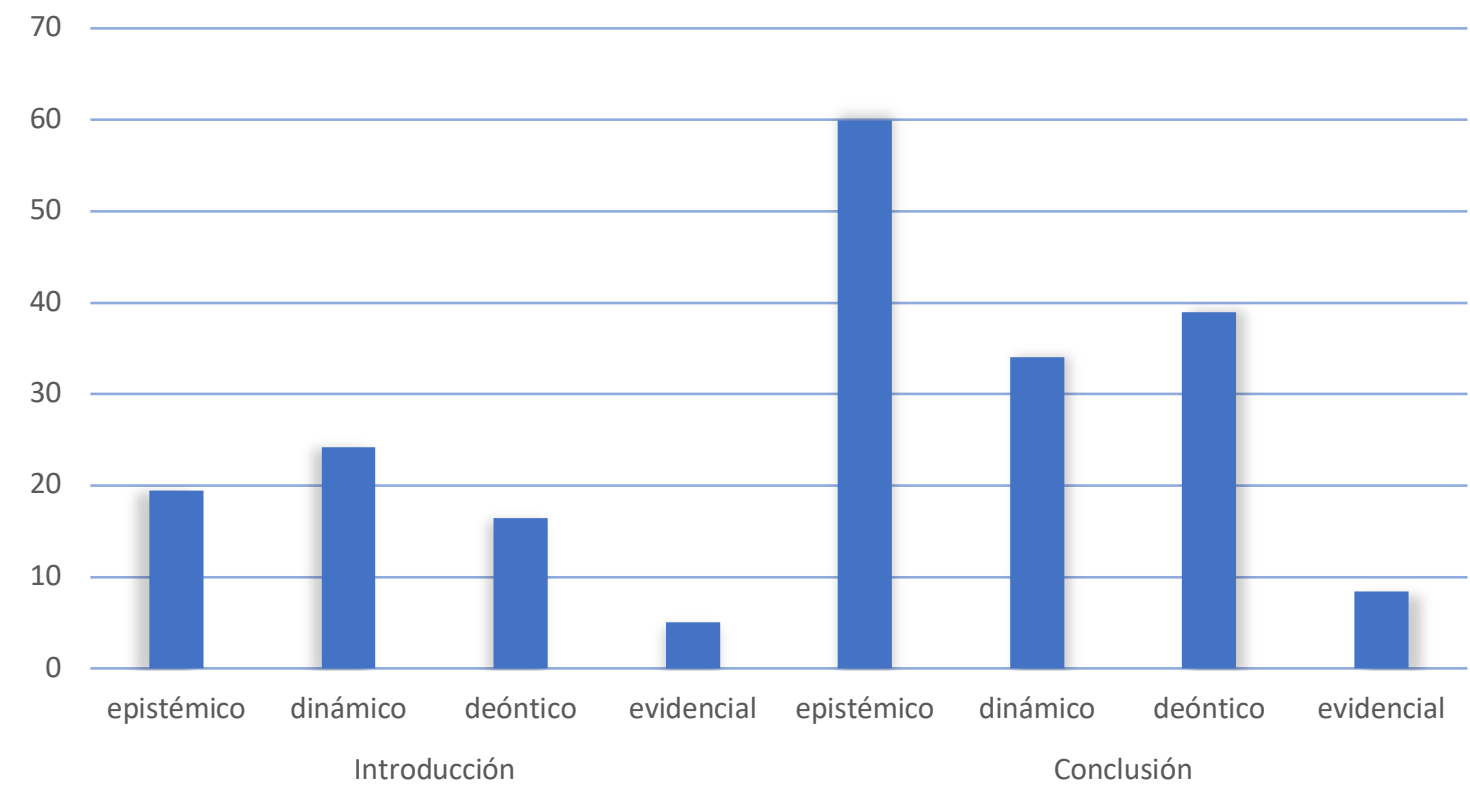

Gráfico 12. Frecuencias relativas de los significados modales por sección.

Como se muestra en este gráfico, mientras que la modalidad dinámica es el significado modal en las introducciones, la modalidad epistémica aparece con mayor frecuencia en las conclusiones seguida de la epistémica y de la deóntica. La modalidad deóntica se usa como segundo recurso modal en las conclusiones seguido del dinámico. La modalidad 
evidencial aparece en las dos secciones con una frecuencia relativa superior en el caso de las conclusiones. Para el cálculo de la variación, usaremos la prueba estadística de loglikelihood (LL), como se ha explicado en la sección 3.2, sobre la metodología de análisis.

Como se hizo en la sección anterior con las formas, la comparación de los resultados de las perífrasis modales encontradas en los análisis con los de Alonso-Almeida y CarrióPastor (2017: 290, 299), se presentan en la tabla 13.

\begin{tabular}{lrrrrrr}
\hline & \multicolumn{3}{c}{ Introducción } & \multicolumn{3}{c}{ Conclusión } \\
\hline & Turismo & Ingeniería & Lingüística & Turismo & Ingeniería & Lingüística \\
\hline epistémico & 19,43 & 34,13 & 12,03 & 59,89 & 27,65 & 98,96 \\
dinámico & 24,21 & 35,23 & 44,42 & 34,03 & 38,29 & 30,45 \\
deóntico & 16,44 & 15,41 & 38,87 & 38,96 & 21,27 & 26,64 \\
evidencial & 5,08 & 0 & 0 & 8,42 & 0 & 0 \\
\hline
\end{tabular}

Tabla 13. Frecuencias relativas de aparición de significados modales por sección en turismo, ingeniería y lingüistica.

Los datos de esta tabla revelan que la modalidad dinámica es el significado preferido en las introducciones de todos los corpus examinados, aunque no hay mucha diferencia entre los significados epistémicos y dinámicos en el caso del corpus de ingeniería. La modalidad epistémica se da con mayor frecuencia en las introducciones de los textos de ingeniería y con menor frecuencia en el corpus de turismo y de lingüística, respectivamente. La modalidad deóntica es más frecuente en las introducciones de lingüística y turismo, en este orden, y con una frecuencia ligeramente menor en las introducciones de turismo. En cuanto a las conclusiones, el significado modal más común es el epistémico en el caso de los textos de lingüística y de turismo. El dinámico es el 
segundo significado modal más frecuente en estas conclusiones en ingeniería y lingüística. En el caso del significado deóntico, este es el segundo modal más usado en el corpus de turismo por razones que se explicarán en el transcurso de este trabajo. Los valores evidenciales solo se encuentran en los textos de turismo, con mayor presencia en las conclusiones.

\subsubsection{Verbos perifrásticos epistémicos}

La noción de modalidad epistémica que se sigue en este trabajo es la formulada por Nuyts (2001: 21), que se ha explicado en el capítulo 2 de este trabajo. Así, la modalidad epistémica se refiere a la "evaluation of the chances that a certain hypothetical state of affairs under consideration (or some aspect of it) will occur, is occurring or has occurred in a possible world". Este sentido de evaluación también se destaca en Cornillie (2009: 47) quien establece que las perífrasis modales epistémicas afectan claramente al contenido proposicional: "epistemic modality evaluates the likelihood that this proposition is true". Esto significa que existe una relación directa entre la noción de verdad y este tipo de modalidad que se relaciona claramente con el conocimiento que se tenga de las cosas. Según estas definiciones, las perífrasis modales epistémicas en el corpus analizado son could, may, might y would. Estas ocurren tanto en las introducciones y las conclusiones, como se observa en la tabla 14.

\begin{tabular}{lrrrr}
\hline & could & may & might & would \\
\hline Introducción & 3,49 & 7,47 & 2,29 & 6,18 \\
Conclusión & 18,16 & 25,37 & 3,97 & 12,39
\end{tabular}

$\overline{\text { Tabla 14. Frecuencias relativas de aparición de significados modales epistémicos por sección en los }}$ artículos de turismo. 
Las frecuencias normalizadas en orden de mayor a menor de estos valores en las introducciones son may, would, could, y might. Algunos ejemplos de estas formas epistémicas son los siguientes:

Chinese tourism demands in Australia have been exponentially increasing in recent years, driven largely by increased economic growth in China, to the extent that the strong growth of this market could be misinterpreted in a way that Chinese visitors are not price-conscious (Introducción; Pham et al. 2018).

(20) The government estimated in the 2015 budget that this measure would bring $\$ 540$ million into the government coffers over the forward estimates (Australian Government, 2015) and this appears to be regarded as sufficient justification for the tax (Introducción; Pham et al. 2018).

(21) Indeed, tourism may be a powerful medium for learning that transforms tourists' habits of thought and ways of engaging with the world (Introducción; Country et al. 2017).

En estos los casos de verbos modales epistémicos, se detectó el valor evaluativo que menciona Nuyts (2001), como se puede observar en los ejemplos (52), (53) y (54). Las conclusiones presentan las mismas formas de transmitir la modalidad epistémica:

(22) This could be due to the lack of access to foods at points during travel or because encounters with unfamiliar foods, foodscapes and rituals reinforced outsider status or social distinctions... Moreover, future research could go further in acknowledging the agency of foods and the 
power of associated practices to transform tourists' conceptions of home and familiarity (Conclusión; Raisi et al. 2018).

(23) The results would be completely different had the segmentation choice been travel motivations (Pesonen, 2012) or general travel activities (Mehmetoglu, 2007) (Conclusión; Pesonen y Tuohino 2017).

(24) This may seem an arbitrary designation from outside the climbing community, but within such subcultural distinctions are used to distinguish hierarchy and award priority at the crags, in campgrounds, and within its political organizations, such as the RRGCC (Conclusión; Rickly 2017).

(25) Extraneous and social-psychological factors, such as weather, company, alcohol consumption might affect visitors' experiences (Conclusión; Andersson et al. 2017).

Las perífrasis modales epistémicas también se usan en la conclusión para expresar evaluación, como se observa en los ejemplos (56), (56), (57) y (58) donde los autores reflejan la probabilidad de que las acciones descritas puedan llevarse a cabo. Como se aprecia, el uso de estas perífrasis modales no es tan diferente en las introducciones y las conclusiones. Sin embargo, existe variación en cuanto a la presencia de este significado en las dos secciones del artículo científico, lo que se comprueba en los valores de la LL presentados en la tabla 15. 


\begin{tabular}{lcccc}
\hline & could* & may* & might & would $^{*}$ \\
\hline Log-likelihood & 101,42 & 95,46 & 4.17 & 19,46 \\
Log ratio & $-2,38$ & $-1,76$ & -0.79 & -1.00 \\
\hline
\end{tabular}

Tabla 15. Valores de log-likelihood y log ratio para medir el efecto de variación de presencia de perifrasis modales epistémicas. El asterisco indica mayor presencia en las conclusiones.

Según los datos de esta tabla, existe variación en el caso de todas las formas epistémicas de acuerdo con su presencia en la introducción y en la conclusión, con mayor frecuencia en las conclusiones como se aprecia en los valores LL. Los datos para el log-ratio, que indica el efecto en el número de veces en el que se dobla la posibilidad en la que puede darse la variación. Esto significa que, en el caso de could, la probabilidad de que esta forma ocurra con este significado en la conclusión es 4 veces mayor que en la introducción. En el caso de would, esto representa que la probabilidad sería 2 veces mayor, pues el log ratio es de 1. El uso más que notable de la modalidad epistémica en las conclusiones, especialmente a través de las formas may, could y would, se explicaría por el hecho de que la función de esta sección es proporcionar interpretaciones provisionales de los fenómenos analizados en el artículo, por lo que el uso de la modalidad epistémica permite reducir la fuerza ilocutiva de la proposición a la que enmarca, como se verá en la sección siguiente.

\subsubsection{Verbos perifrásticos con significado evidencial}

Aunque con frecuencia la modalidad evidencial se sitúa como un subdominio de la modalidad epistémica (véase Palmer 1986), aquí la hemos tratado de manera separada, pues, si bien hace referencia a cuestiones de información, su uso designa la manera en la que se ha obtenido dicha información. Los verbos que se usan con este valor en nuestro 
corpus son could, may, might, would y should. La tabla 16 presenta las frecuencias de estas formas.

\begin{tabular}{lccccc}
\hline & could & may & might & would & should \\
\hline Introducción & 0,20 & 2,29 & 2,29 & 0,20 & 0,10 \\
Conclusión & 0,48 & 6,37 & 0,96 & 0,48 & 0,12 \\
\hline
\end{tabular}

Tabla 16. Frecuencias relativas de aparición de perífrasis con significado evidencial por sección en los artículos de turismo.

Como se aprecia en esta tabla, la forma más frecuente es may en la conclusión, mientras que may y might son las formas más frecuentes en la introducción. Un ejemplo de este tipo de uso modal es may y could en los ejemplos siguientes:

(26) Given this change in policy, this research note examines the degree of persistence in US tourist arrivals, over the entire period in which data are available from January 1996 to August 2016 (which encompasses the period for the Cunado et al. (2008a) study) along with two subperiods; January 1996 to August 2001 and September 2001 to August 2016, in order to determine the extent to which the change in data measurement associated with the requirement of the INS I-94 entry form may have impacted the degree of persistence (Introducción; Payne y Alana 2018). Rather than seeing these limits as an example of transformation that could have been done better, we argue that understanding limits is an important part of the experience for visitors who are engaging in complex issues underpinned by very real ontological differences (Conclusión; Country et al. 2017). 
En estos ejemplos, las perífrasis modales may y could aparecen como parte de un texto expositivo para contextualizar el razonamiento inferencial que se produce en estos textos. Todo ello está convenientemente señalado por los marcadores de discurso como in order to, siendo este último la forma lingüística que, posteriormente, permite introducir el enunciado en el que surge may have impacted en el caso en (59), y rather than seeing en el caso en (60) donde se manifiesta necesidad epistémica (véase Van der Auwera \& Plungian 1998). Este tipo de construcciones representa lo que Boye y Harder (2009) describen como sustancia evidencial (evidential substance), esto es, un modal seguido de una forma perfectiva (have + participio) o progresiva (be + v-ing), de cuya función se hablará en la sección 4.5.

La tabla 17 presentan los valores para la evaluación de la variación que existe entre la frecuencia de los modales con significado evidencial en cada una de las secciones.

\begin{tabular}{lrrrrr}
\hline & could* & may* & might & would* & should* \\
\hline Log-likelihood & 1,11 & 18,48 & 5,02 & 1,11 & 0,02 \\
Log ratio & $-1,27$ & $-1,48$ & 1,25 & $-1,27$ & $-0,27$
\end{tabular}

Tabla 17. Valores de log-likelihood y log ratio para medir el efecto de variación de presencia de perífrasis modales con significado evidencial. El asterisco indica mayor presencia en las conclusiones.

Como se aprecia en esta tabla, las formas presentan variación en su uso en las dos secciones con mayor frecuencia en las conclusiones, excepto en el caso de might. La forma que presenta mayor variación es may con un LL de 18,48 y un log ratio que permite interpretar que la variación representa más del doble en el caso de la conclusión con respecto a la introducción. Esto mismo sucede con may y would. Sin embargo, en el caso de might, esto ocurre al contrario, pues presenta mayor probabilidad de frecuencia de aparición de esta perífrasis en la introducción frente a la conclusión. La forma que menos 
variación ofrece es should con un $L L$ de 0,02 y un log ratio bajo, esto es 0,27 , que indica una escasa probabilidad de variación entre las dos secciones.

\subsubsection{Verbos perifrásticos dinámicos}

Las perífrasis modales dinámicos utilizadas en las introducciones de los textos son can, could, may, will y would que presentan la distribución que se indica en la tabla 18.

\begin{tabular}{lccccc}
\hline & can & could & may & will & would \\
\hline Introducción & 23,32 & 0,00 & 0,10 & 0,70 & 0,10 \\
Conclusión & 33,67 & 0,24 & 0,12 & 0,00 & 0,00
\end{tabular}

Tabla 18. Frecuencias relativas de aparición de significados modales dinámicos por sección en los artículos de turismo.

Se muestra tanto en la tabla como en el gráfico que, sin lugar a duda, can tiene los valores dinámicos más altos tanto en la introducción como en la conclusión. En el caso de las conclusiones, los sentidos dinámicos también aparecen en este mismo conjunto de verbos, aunque con una frecuencia muy diferente. La proporción LL revela que la presencia de los modales dinámicos es, en efecto, muy significativa, como mostraremos a continuación. En ambas secciones, el modal más frecuente es can. En los ejemplos (28) a (31), a continuación, se dan casos de esta modalidad en contexto, donde can implica características que son específicas y se refieren al objeto al que se refiere en cada caso:

(28) Thus, based on the IP addresses of consumers, a hotel company can advertise a given price in a particular country but not make that price available to users from other countries (Introduction; Song et al. 2018). 
(29) Some notable examples include Cooper et al. (1998), Goeldner and Ritchie (2003), and Hudson (1999), each questioning how a model can predict travel behavior as tourists travel with different motivations on different occasions (Introduction; Litvin y Smith 2016). Representational performances can offer a venue to reimagine how the myth is presented, and ask important questions that should be asked of all national myths (Conclusion; Zhang y Ryan 2018).

(31) This paper has provided an importance index and identified the 30 most important web-sites within the West Australian tourism industry. This can assist key policy makers and managing bodies of the destination to have a better understanding of important hubs, where they are located in the network, and how their structural powers can be used for the better management of the network. Moreover, it can help hubs to better understand their position, and more strategically plan their networking on the Internet (Gardiner y Kwek 2017).

En estos ejemplos, Las cualidades de los objetos this ('an importance index'), company, examples, performances y structural powers que aparecen como sujetos del modal can en cada caso permiten entender que las acciones se llevan a cabo gracias a estas propias cualidades. En ocasiones, se puede entender la existencia de algunos matices epistémicos en el uso de can, ya que también puede deducirse cierto grado de probabilidad de que la proposición precedida por can se realice. Esto depende de cada hablante y qué premisas contextuales se tengan en cuenta en la interpretación de la forma modal. Un ejemplo claro podría ser el último caso de can en (31), pues se puede juzgar que can indica la 
probabilidad que existe de que an importance index sea de ayuda, en vez de que esto tenga solo la capacidad de ayudar. En la segunda interpretación, se refiere a un valor dinámico que habilita la acción descrita.

Este mismo significado de posibilidad dinámica para llevar a cabo una acción se encuentra en el uso de las perífrasis modales, como se ejemplifica en los ejemplos (32) y (33) con will y may, respectivamente. El sentido de futuro en (32) es ciertamente inevitable, ya que la afirmación se asemeja a la formulación de una promesa en el sentido en que se describe en Searle (1969). En este sentido, la voluntad de querer llevar a cabo la acción parece indicar que se cumplirán las condiciones necesarias para esto. En (33), la forma may indica el resultado lógico resultante de lo que se ha dicho anteriormente en el texto y, por lo tanto, su uso indica la capacidad del sujeto para realizar la acción.

(32) The successful incorporation of AR into the tourism model will contribute to understanding the rapidly evolving technologies travelers will face in the future (Introducción; Chung et al. 2018).

(33) To continue with this line of research, we suggest to study whether the presence of foreign direct investment in the hotel industry gives rise to an increase in the competitiveness of the destination of the investment, due to knowledge transfer. Accordingly, efficiency may explain the reverse direction of causality (Conclusión; Mendieta-Peñalver et al. 2018).

La variación en el uso de la modalidad dinámica en las introducciones y conclusiones se presenta en términos del cálculo de LL en la tabla 19. 


\begin{tabular}{lccccc}
\hline & can* & could* & may* & will & would \\
\hline Log-likelihood & 17.31 & 3,17 & 0.02 & 8,45 & 1,21 \\
\hline Log ratio & -0.53 & $-2,27$ & $-0,27$ & inf. & inf. \\
\hline
\end{tabular}

Tabla 19. Valores de log-likelihood y log ratio para medir el efecto de variación de presencia de perifrasis modales epistémicas. El asterisco indica mayor presencia en las conclusiones.

Como se muestra en esta tabla, la modalidad dinámica parece ser más frecuente en las conclusiones de los artículos de investigación en turismo. Los datos obtenidos del cálculo de LL indican que las formas can, could y may son más probables en las conclusiones, posiblemente porque permiten la presentación de ideas sin imponer de manera directa el criterio de los autores. Sin embargo, las perífrasis will y would con sentido epistémico solo aparece en las introducciones y no en las conclusiones, por lo que no se obtienen datos de log ratio, de ahí "inf." en la tabla 19.

\subsubsection{Verbos perifrásticos deónticos}

La modalidad deóntica es la menos frecuente de los tres tipos de modalidad en los textos que se han analizado. Las perífrasis que se han identificado en las introducciones y las conclusiones se ofrecen en la tabla 20.

\begin{tabular}{lcccccr}
\hline & can & could & must & will & shall & should \\
\hline Introducción & 1,10 & 0,00 & 1,00 & 10,46 & 0,40 & 3,49 \\
Conclusión & 0,72 & 0,48 & 3,37 & 15,75 & 0,24 & 18,40
\end{tabular}

Tabla 20. Frecuencias relativas de aparición de significados modales deónticos por sección en los artículos de turismo.

Como se indica en esta tabla, la modalidad deóntica se expresa por medio de las formas modales can, must, will, shall y should, en el caso de las introducciones, y can, could, 
must, will, shall y should en las conclusiones del corpus. Los siguientes son ejemplos con will extraídos del corpus donde se puede intuir nociones de necesidad y de predicción o, incluso, expectativa.

(34) Doing this will allow for a more detailed comparison between different discourses on a particular issue, leading to a better understanding of the nuanced relations between different social agents (Xue y Kerstetter 2018).

(35) The complexity of the voluntourist event can be seen from multiple and unexpected perspectives and our contribution will (we hope) support future re/theorisation of an increasingly popular and commodified activity (Bone y Bone 2018).

Los siguientes ejemplos contienen otras formas de perífrasis deónticas como must, should y shall, cuyos significados giran en torno a sentidos de necesidad y la obligación:

For a relationship between the tourism destination and AR to truly exist, the incorporation of $A R$ and a place must collectively affect a traveler (Introducción; Chung et al. 2018). Performing arts managers must refine the service concept, the service marketing direction and the market positioning strategy as being essential components of the service product strategy for the performing arts. This service strategy must include focus on the peripheral aspects of the entire service experience (Conclusión; Song 2016). Unfortunately, there is often a social expectation that people should stay connected to work after hours or on vacation, making it 
challenging for people to distance themselves from work demands and stress (Introducción; Chen et al. 2018).

(39) This research agenda should be informed by primary data collected from hoteliers and peak industry bodies specifically for the purpose of developing robust future hotel employment scenarios and analysed using advanced foresight techniques in order to address the limitations of the current exploration (Solnet et al. 2016).

En todos estos casos de perífrasis deóntica, la idea de necesidad es un valor clave de los modales deónticos en las conclusiones. Charlow (2016: 47 y sig.) menciona la tradicional división de los modales según la necesidad en modales de necesidad débiles y fuertes. La forma modal must correspondería al grupo de modales de necesidad fuerte en los ejemplos (36) y (37), mientras que la forma should en los ejemplos (38) y (39) se clasificaría como un modal de necesidad débil. La autora afirma, sin embargo, que no existe un modal de necesidad fuerte, por lo que resuelve "to treat strong deontic necessity as a special case of weak deontic necessity" (Charlow 2016: 47).

Se incluyen los valores de LL y de log ratio para los sentidos de modalidad deóntico en las introducciones y en las conclusiones en la tabla 21.

\begin{tabular}{lcccccc}
\hline & can & could & must* & will* & shall & should* \\
\hline Log-likelihood & 0,7 & 6,33 & 12,6 & 9,84 & 0,36 & 103,76 \\
Log ratio & 0,6 & $-3,27$ & $-1,76$ & $-0,59$ & 0,73 & $-2,4$ \\
\hline
\end{tabular}

Tabla 21. Valores de log-likelihood y log ratio para medir el efecto de variación de presencia de perífrasis modales deónticas. 
Los datos que se reflejan en esta tabla sugieren que could, must, will y should parecen tener mayor probabilidad de presentar variación en las conclusiones que en las introducciones. En este sentido, la forma could muestra un efecto mayor de que esto ocurra, esto es 3,27, que indica que la probabilidad de aparición de este sentido modal es seis veces mayor en el caso de las conclusiones. En el caso de should, el log ratio indica que esta probabilidad es de casi cuatro veces y de dos veces en el caso de must, frente a will cuyo efecto es realmente pequeño. Esto mismo ocurre con can y con shall, cuyos valores reflejan que existe variación en cuanto a su uso, si bien el efecto es pequeño, no llegando a ser doble la probabilidad de que esto suceda. Con todo, shall presenta la probabilidad más alta de aparecer en la introducción que en la conclusión.

\subsection{Las funciones de las perífrasis modales}

Sin lugar a duda, las perífrasis modales tienen como función expresar la perspectiva del hablante con respecto al contenido proposicional. Si bien este valor de la modalidad refleja, en gran medida, la propia evaluación del hablante no es la única finalidad de un elemento modal. Por el contrario, el uso de estos modales manifiesta una intención específica que se entiende gracias al contexto donde se produce el acto de habla en concreto. Entre otras, se distinguen estos usos en el corpus de introducciones y conclusiones de los artículos científicos: (a) función de mitigación y cortesía lingüística, (b) función predictiva, (c) expresión de la factualidad, (d) significado inferencial, (e) indicación de la organización, (h) expresión de la autoridad, (i) imposibilidad de realizar una acción, (j) formulación de recomendaciones a autores, y la (k) formulación de recomendaciones dirigidas al sector turístico. En lo que sigue se describen estas funciones con ejemplos tomados del corpus. 


\subsubsection{Mitigación, duda y cortesía lingüística}

El uso de las perífrasis modales como mecanismo de cortesía lingüística para atenuar el contenido proposicional es una de las funciones de estas formas modales. En los siguientes ejemplos, se emplea la forma modal could con esta función tanto en las introducciones y las conclusiones:

(40) ...because it could help them develop more appropriate and competitive marketing strategies to attract new guests while ensuring repeat business from existing ones (Introducción; Alnawas y Altarifi 2016).

(41) What is more, even though some authors claim consumers to represent 'rational utility maximizing agents' (Sampaio et al., 2012: 235), others point out that consumers could actually be persuaded to engage in more sustainable behaviour, for instance, by using subtle cues (e.g. see De Groot and Steg, 2007) or strengthening relevant normative goals (Conclusión; Melissen et al. 2016).

(42) If we assume that online chatting with friends is not entirely unlike people sharing their travel experiences around a kitchen table, we could argue that SNSs just give us easy access to how it is done in practice (Conclusión; Alnawas y Altarifi 2016).

El uso could en los ejemplos (403) y (41), could help them develop y could actually be persuaded (42) son ejemplos de formas epistémicas con las que se pretenden modular el contenido proposicional para evitar la imposición del punto de vista de los autores. En el caso de could en (42), could argue, no sería un ejemplo de modalidad epistémica sino 
dinámica, aún así se trataría de una estrategia de cortesía negativa. Los autores mitigan el contenido proposicional mediante la manifestación del contexto que expresa la perífrasis modal could, que indica que se dan las circunstancias que habilitan a los autores estar 'en posición' de argumentar sobre lo expresado en la cláusula introducida por that. La diferencia con el resto de los ejemplos es que el uso de could no parece indicar duda o ausencia de certeza, como se apunta en Alonso-Almeida (2021).

En los siguientes ejemplos con may, se percibe la intención de los autores por evitar la imposición de su punto de vista, por lo que se tratarían de mecanismos de cortesía negativa:

(43) The expanding nature of branding strategies and corporate culture is an example of how the Authorised Heritage Discourse (Smith, 2006) cannot be linked to a 'top-bottom' dichotomy but rather to a heritage regime that is being naturalised as the unquestioned way of being in the world (Alonso González, 2013; Bendix et al., 2012). It is not just a matter of an elitist discourse that may (or may not) be assumed by local population (Introducción; Jiménez-Esquinas y Sánchez-Carretero 2018).

(44) Thirdly, with the use of student sample, the generalizability of the results is decreased since the views held by students toward Pakistan may not be representative of the general population of their respective countries. Moreover, the financial ability of the students may be limited, which restrict their future intentions to visit another country (Hsu and Sung, 1997) (Conclusión; Yousaf y Samreen 2016). 
Como ocurre con varias formas de could descritas anteriormente, todos los casos de may en (43) y (44) muestran un uso mitigador del significado de las proposiciones a la que acompañan. Si bien es cierto que estas formas, parecen indicar cierta duda o falta de certeza por parte de los autores, esto puede ser simplemente una estrategia de cortesía, sin que necesariamente signifique que los autores no están convencidos de la realidad que están describiendo. La perífrasis modal might también se usa con una función atenuadora en los siguientes ejemplos:

(45) From the outside, this juxtaposition might seem quite odd, being part of the individual way of the Romanians to make a travail de mémoire (Introducción; Banica 2016).

(46) After mutual selection, the couchsurfers who share the same value and perform properly online are selected to the stage of offline performance, while others might be excluded from the face-to-face interaction of Couchsurfing... To avoid a negative reference, couchsurfers create a backstage to hide the "improper behaviours" which might conflict with the social conventions of Couchsurfing (Conclusión; Chen 2018).

En todos estos casos de might en (45) y (46), existe una dimensión evaluativa con la que se indicaría la probabilidad de que la acción descrita en la proposición pueda cumplirse. Como señala Collins (2009: 92), mediante estos usos, se da a entender una "lack of confidence in the truth of the proposition", y este es, en efecto, lo que Hyland refiere como hedging, pues estas permiten presentar "a proposition as an opinion rather than a fact" (Hyland 1998: 5). En definitiva, el uso de estas estrategias se entendería como una declaración de la falta de compromiso de los autores con la proposición expresada 
(Coates 2003: 334), aunque esto no es necesariamente así, como se explicó con anterioridad. En términos de cortesía, se clasificarían, por lo tanto, como estrategias de cortesía negativa para evitar imponer la perspectiva de los hablantes.

\subsubsection{Función predictiva}

Las perífrasis modales con significado deóntico se usan para expresar predicción en las introducciones y en las conclusiones, como se observa en el ejemplo que se ofrece a continuación:

In drawing out the reciprocities between travel motivations and their subsequent manifestations, more light will be shed on backpackers' needs and sought benefits and the circumstances of their potential fulfilment. Both scholars and practitioners alike can benefit from an increased transparency that contributes to our insight into this visitor type. Results of the study will challenge previous interpretations of how travel motiva- tions such as interests in foreign cultures and sociability are fulfilled through social behaviour and will simultaneously illustrate how social encounters can influence the perception of tourism products, attractions and services, thus offering a new perspective on the assigned importance of the social element in the context of visitor satisfaction (Introducción; Reichenberger 2017).

Este ejemplo representa parte de una introducción de un artículo sobre turismo para mochileros. El uso de la perífrasis modal will con significado deóntico y con una función predictiva persigue un efecto de presentación de los contenidos, por una parte, y un 
efecto promocional, por el otro. Esto es especialmente evidente en Results of the study will challenge previous interpretations... and will simultaneously illustrate... donde se origina expectación al lector ante la promesa que refleja los usos de esta perífrasis modal en este contexto.

Lo mismo ocurren en la conclusión, como se observa en el siguiente ejemplo: Imposing a 10-year visa fee of $\$ 1000$ for Chinese visitors is a risky approach if this scheme replaces the existing 3-year visa fee, as this increase will erode the demand from the largest market of the Australian inbound sector. It will effectively defeat the efforts that other reforms have made. The range of negative impacts is large for the tourism sector to overcome. This will raise a question as to whether or not the government is fully committed to the Potential target that both the government and the industry have set up. Changes to conditions of visa application such as administrative procedures, complexity of requirements and fees and charges could restrict or open up market access for foreign nationals (Liu and McKercher, 2014). Consequently, this will lower business confidence in the sector, could affect the long-term investment strategy of investors and potentially be detrimental to trade and inflow of foreign direct investment (Neumayer, 2011; Song et al., 2012) (Conclusión; Chen 2018).

En este ejemplo, el uso de will tiene la misma función de evaluar la situación resultante de un contexto donde se cambie el sistema actual de un visado de tres años para los 
visitantes chinos en Australia por uno de diez años con un coste de diez mil dólares. Los autores relacionan las consecuencias de este cambio en un hipotético escenario que refleja con will y sustenta mediante el empleo de ciertos elementos textuales que indican resultado (as, consequently), además de la perspectiva de los hablantes sobre los hechos que se concluyen (effectively). Una representación diagramática la podemos ver en el gráfico 13:

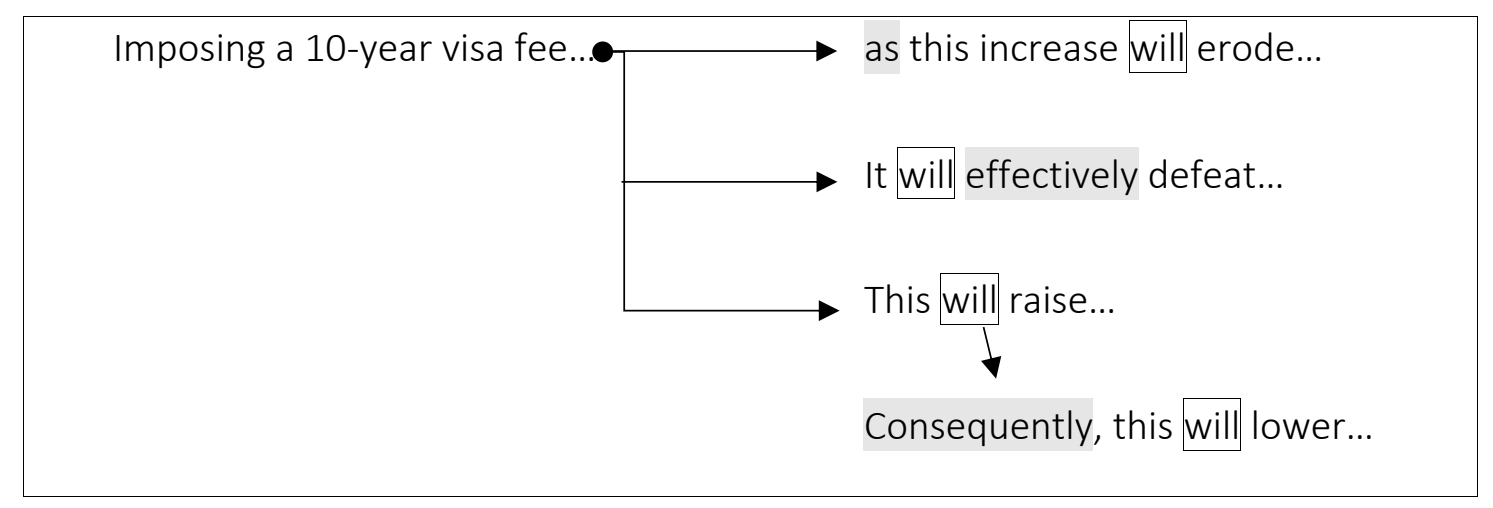

Gráfico 13. Representación de la predicción a partir de la premisa partida.

Estos valores deónticos de predicción, especialmente en la conclusión de los artículos de investigación, suelen tener también cierto valor de resultado que sucede a modo de conclusión del trabajo presentado, como ocurre en los ejemplos siguientes: Taking a gap year involves physical mobility to a periphery destination, which will lead to social mobility after returning to the gapper's centre (Conclusión; Hernmann et al. 2017).

$$
\text { For example, to overcome the security concern that is hindering }
$$
Internet adoption, TAs in Dubai can form alliances in order to develop a more secure and effective transaction-based website. Additionally, creating alliances with other industry facilitators such as airlines, tour 
operators, hotel chains, international TAs and cruise lines will certainly increase their competitiveness and their market share. Another significant barrier that may be hindering e-commerce adoption is the lack of IT skilled labour. This could be overcome by sending employees in TAs to attend professional training courses and sessions related to ICT (Conclusión; Zaidan 2017).

En esto ejemplos, la forma modal will se combina con elementos léxicos que indican razonamiento lógico como lead to en (49) y las estructuras adverbiales como for example y additionally en (50) que contribuyen a este sentido de resultado de la investigación sustentado por las evidencias que se han considerado en la elaboración del conocimiento. Siguiendo esta misma línea de razonamiento, en este mismo ejemplo, se explicaría el adverbial evidencial certainly, que refuerza el punto de vista de los autores, así como la estructura evidencial introducida por la perífrasis may seguida de aspecto progresivo may be hindering que se remata en el texto con la sugerencia presentada con la forma modal could, en This could be overcome.

\subsubsection{Función de expresión de la factualidad}

Desde una perspectiva pragmática, el uso de las perífrasis modales dinámicas en las conclusiones puede tener un efecto fortalecedor en la comunicación, ya que este significado modal hace referencia a un hecho verídico basado en la presunción de que una persona, un evento o un objeto tiene la capacidad intrínseca o se dan las condiciones externas para que realicen la acción descrita en la proposición. De ahí que la información se ofrezca como una conclusión. Sin embargo, aún así, estas perífrasis pueden mitigar la 
fuerza elocutiva del contenido proposicional (véase Depraetere (2017: 16)), ya que los hechos expresados se basan en hechos potencialmente posibles y/o habilidades. Algunos ejemplos son los siguientes:

Este es el caso de can y may en los ejemplos siguientes:

(51) Destination image in relation to tourism can be defined as a continuous mental process by which one holds a set of impressions, emotional thoughts, beliefs, and prejudices regarding a destination due to information obtained from different channels (Crompton, 1979; Liou, 2010; Milman, 2011; Reynolds, 1965) (Introducción; Kim y Chen 2016).

(52) In particular, we highlight works that can help us to use a (critical) mobility lens to sharpen our understanding of the forces shaping the development of these policies as well as the implications thereof (Introducción; Torabian y Mair 2017).

(53) Such research directions can facilitate the development of important research streams, e.g. segmentation, latent class/profile analyses), which together can provide the theoretical insight that is necessary to explicate the differential adoption patterns of various population groups (Conclusión; Morosan 2018).

(54) Photos and visual processing may, after all, be more basic to human existence than the processing of verbal information, and photos may therefore evoke deeper elements of consciousness than words (Harper, 2002) (Conclusión; Andresson et al. 2016). 
En el ejemplo (51), la perífrasis can se usa para introducir una definición, por lo que, en absoluto, expresa duda. Por el contrario, hace referencia a una posibilidad real y verídica que representa al concepto destination image in relation to tourism. En realidad, en este contexto, podría no ser necesario el uso de esta forma modal, al igual que ocurre en el resto de los ejemplos, ya que hacen referencia a un hecho cuya posibilidad radica en las características internas de los sujetos o a las condiciones que, en efecto, se dan. La presencia de estas perífrasis, como se indicó anteriormente, se mantiene para contextualizar la información evitando la imposición (Brown \& Levinson 1987) de manera explícita de la perspectiva y previene, así, críticas futuras en la literatura científico. En el caso de las otras formas de can en los ejemplos (52) y (53), también hacen referencia a la posibilidad real fundamentada en la capacidad que tienen los objetos o eventos mencionados, works en (52), such research direcctions en (53) y research directions y research streams también en (53), para realizar las acciones descritas en cada una de las proposiciones precedidas por la forma modal. En el caso del ejemplo en (54), may en las dos ocasiones en la que aparece expresa la posibilidad de que se lleven a cabo las acciones escritas basándose en las cualidades de los sujetos responsables de estas acciones.

\subsubsection{Inferencialidad}

El uso de las perífrasis modales para expresar significado inferencial mediante la combinación con el aspecto perfectivo o el aspecto progresivo ha sido identificado como un mecanismo recurrente en la retórica de los textos científicos, como se indica en los trabajos de Alonso-Almeida (2015b) y Alonso-Almeida y Carrió-Pastor (2017), entre otros. En su estudio sobre los modales suecos, Beijering (2017: 53) describe este tipo de 
construcciones como la representación de un razonamiento lógico a la luz de las pruebas existentes, como también se aprecia en los ejemplos siguientes:

(55) Given this change in policy, this research note examines the degree of persistence in US tourist arrivals, over the entire period in which data are available from January 1996 to August 2016 (which encompasses the period for the Cunado et al. (2008a) study) along with two subperiods; January 1996 to August 2001 and September 2001 to August 2016, in order to determine the extent to which the change in data measurement associated with the requirement of the INS I-94 entry form may have impacted the degree of persistence (Introducción; Payne y Gil-Alana 2018).

(56) Moreover, the Greek government rejected a one-million euro offer from the brand Gucci to host a fashion show at the Acropolis in Athens, which could have aided the government's restoration efforts (New York Times 2017) (Introducción; Biraglia et al. 2018).

(57) Given the complexity of the contexts involved (of which I have only offered an outline), it is hardly surprising that the representation of tourism should have taken on a variety of shapes and meanings (Conclusión; Sampaio 2017).

(58) Additional participants could have been sought, but our analysis suggested that findings were converging and that little variation would be achieved with additional data (Conclusión; Cheok et al. 2016). 
En estos ejemplos, la función pragmática de lo que Boye y Harder (2009) ha denominado evidential substance es atenuar el contenido de la proposición, así como para ofrecer el punto de vista de los escritores y su participación en la elaboración del significado, como destaca Alonso-Almeida (2015a). En otras palabras, se trata de utilizar una estrategia de cortesía negativa para evitar la imposición a los destinatarios del mensaje (Brown \& Levinson 1987: 70; Haugh, Kádár \& Mills 2013: 18). Esto se logra cuando los autores comparten su propio razonamiento en el proceso de investigación con las estructuras inferenciales may have impacted (55), could have aided (56), should have taken (57) y could have been sought en (58). Con esto, los autores informan a los lectores y a las lectoras tanto de su implicación real en la elaboración del conocimiento, como de la naturaleza cognitiva del mismo reduciendo así la fuerza de la imposición en cierta manera al revelar que se trata de un proceso deductivo y, como tal, subjetivo, por lo que está sujeto a validación.

\subsubsection{Indicar la organización}

Uno de los usos más evidentes de la modalidad es la indicación de la organización del artículo de investigación, en tanto que su empleo radica en manifestar la intención con respecto a la evolución del propio trabajo, como se muestra en los ejemplos siguientes:

(59) I will discuss the conceptual, theoretical relations between visitor experience and the idea of the heritage destination in the next section, "Heritage destinations." Thereafter, I will use the example of a Freedom Park tour to show how a particular narrative journey was used to frame ideas about the site's South African and African distinctiveness and how tourists actually engaged with such claims (Introducción; Jethro 2016). 
(60) In doing so, this study will be divided into five sections (Introducción; Bruttomesso 2018).

(61) Accordingly, the primary objective of this article is to combine four distinctive and unique constructs (i.e. brand identity, brand-lifestyle congruence, $\mathrm{CHBI}$, and brand love) into one model to delineate the consumer's psychological path to brand loyalty in the hotel industry and to test its predictive power in explaining brand loyalty (see Figure 1). The rest of the article is structured as follows: the second section provides a theoretical background on the key constructs of the current study, while the third section proposes the framework of the current study and develops the research hypotheses. This will be followed by analyzing and testing the research hypotheses. The last section concludes the findings. Managerial implications and direction for future research are also provided (Introducción; Alnawas y Altarifi 2016).

(62) We shall first provide a theoretical discussion that acknowledges how destinations are mobile entities and in a constant state of becoming. We then will provide an historical overview of the destination image of Iceland suggesting that the changing image of Iceland is crucial to understanding the present state of affairs (Introducción; Lund et al. 2017).

En los ejemplos anteriores, se muestran usos de will y shall con valor deóntico que persiguen mostrar la estructura del trabajo y la voluntad de incluir ciertos contenidos. Este tipo de información suele darse lógicamente en la introducción, lo que daría 
respuesta a varias de sus funciones que consisten en la presentación de los objetivos y el planteamiento del propio estudio. En el ejemplo (59), will indica la voluntad de los autores en comunicar qué aspectos se van a tratar en el texto. La indicación temporal predomina para marcar el orden en el que estos aspectos se sucederán a lo largo del artículo de investigación. En este mismo ejemplo, esto se marca, además, por los adverbiales thereafter and in the next section, mientras que, con la misma función, aparece while en (61), and first and then se emplean en (62) para reforzar este aspecto cronológico. El sentido de voluntad en cuanto a la estructura se percibe bien en el ejemplo (60), donde la perífrasis deóntica will indica la intención estructural del artículo, esto es be divided into five sections. Estas formas modales combinan con formas de presente para lograr el fin de presentar la organización del artículo, como se observa en el ejemplo (61): is... is structured... provides... proposes... will... concludes... are provided...

Finalmente, en el ejemplo (62), la combinación de shall en la primera línea y de will en la estructura formularia de carácter intersubjetivo we + (will/shall) + provide + SN sugiere no solo que estas formas modales tienen un valor semejante, sino que su uso puede estar determinado por cuestiones de estilo y no por su significado o por su función. Con esta fórmula se consigue también dar textura al texto mediante su repetición, lo que parece ser una función importante de los elementos modales, como apuntan Alonso-Almeida y Carrió-Pastor (2017) y Alonso-Almeida y Álvarez-Gil (2021b).

\subsubsection{Expresión de la autoridad}

A pesar de que existe una conciencia por parte de los autores por mitigar sus afirmaciones para evitar críticas por parte de la comunidad científica ante una aseveración directa que puede entenderse como una imposición (Martín-Martín 2008; Carrió-Pastor 2012; 
Czerwionka 2012; Loi, Lim \& Wharton 2016; Carrió-Pastor 2017), es igualmente necesario mostrar el grado de implicación como especialista para garantizar la fiabilidad de la información que se presenta en el ámbito científico. Los ejemplos siguientes ilustran este aspecto:

(63) Tourism scholars must question the ways in which travel policies and programmes work 'to determine the mobility of those people whose "credentials" to travel are often deemed "risky," "suspect" or "illegitimate"' (Bianchi and Stephenson, 2014: 98-99) and to unpack the ideological forces shaping the development and normalization of those policies and programmes (Introducción; Torabian y Mair 2017).

(64) Despite the voluminous literature devoted to the relationship between pilgrimage and tourism, there is a disagreement in what constitutes the difference between pilgrimage and tourism from the religious perspective, the perspective of the pilgrims themselves, and the perspective of the tourism industry and researchers (Timothy and Olsen, 2006). I shall not make another inventory of the resemblances and differences between them for the Romanian case, among other things because the state of scientific research on this subject in Romania is almost non-existent (Conclusión; Banica 2016).

(65) These experiences should not be understood as discrete human and non-human actors provoking two passive visitors, but rather as an act of more-than-human engagement and co-constitution (Conclusión; Country et al. 2017). 
although they are widely used, they are scarcely formally planned; and mobile applications must be considered in a stage 1 of development, as its low use is combined with low perceived usefulness by DMO managers (Conclusión; Fernández-Cavia et al. 2017).

Como se ha sugerido anteriormente en la descripción del marco teórico, la expresión de autoridad puede realizarse de manera directa mediante el uso del imperativo o mediante las perífrasis modales deónticas. En los ejemplos anteriores, las formas modales usadas son must en (63) y (66) y shall y should en (64) y (65), respectivamente. En el caso de must en (63), se usa para expresar lo que parece ser una obligación a la comunidad científica para que se plantean cuestiones destinadas a la mejora, lo que los autores plantean tras el análisis de situación. En efecto, queda patente el sentido de autoridad que, además, queda reforzado mediante el uso de mecanismos de atribución, reflejados aquí por la referencia bibliográfica, que apoya la perspectiva del hablante.

En los ejemplos (64) y (65), la idea de autoridad surge por la combinación de la forma modal con el adverbio de negación not: shall not y should not. Se ha demostrado que este adverbio modifica el significado de la forma modal o del contenido proposicional (véase de Haan 1997). En los casos de shall not, se indica que hay intención de no llevar a cabo la acción descrita en la proposición: shall [NOT (make another inventory)]. En el caso de should not, el adverbio de negación parece llevar a cabo la misma función, aunque los sujetos que conceptualizan esta obligación, en este caso los autores, permanezcan implícitos: should [NOT (these experiences) (make another inventory)]. 
Otra manera de mostrar autoridad en los textos académicos es mediante el uso de la matriz it should be noted, como en los ejemplos siguientes:

(67) It should be noted that the literature on needs and motivation is ample; however, for the purposes of the current study, only those seminal conceptual pieces and the studies specifically developing a needs or motivation measurement tool are reviewed here (Introducción; Tasci y Ko 2017).

(68) Third, though authenticity has served as a powerful analytical concept, it should be noted that in the discursive field, the different types of authenticity are usually inseparable in a topical conversation (Conclusión; Wang y Alasuutari 2017).

En los ejemplos (67) y (68), la matriz ya mencionada muestra un significado modal efectivo (effective modality), siguiendo la terminología en Marín-Arrese (2009) cuya intención es la de manifestar necesidad, consejo u obligación. En este sentido, el contexto en los ejemplos (67) y (68) parece indicar la necesidad y la conveniencia de considerar lo que se describe en las oraciones con that. En otras palabras, las matrices en los dos casos que se analizan aportan la evaluación subjetiva de los autores, si bien estos aparecen implícitos en la configuración de estas fórmulas como los sujetos de donde procede la fuerza deóntica.

\subsubsection{Imposibilidad de realizar una acción}

Las perífrasis modales se usan en el corpus analizado para expresar la imposibilidad de llevar a cabo la acción que se describe en la proposición. Dicha imposibilidad puede ser 
debido a la acción de una fuerza externa o interna en el caso de las perífrasis deónticas, mientras que, en el caso de las perífrasis dinámicas, esto ocurre por la falta de habilidad, capacidad o por la ausencia de condicionantes que permitan la consecución de la acción contenida en la proposición. Algunos ejemplos del corpus son los siguientes:

It cannot, however, be denied that new patterns of tourism-related consumption were also emerging during these years (Introducción; Sampaio 2017).

(70) We simply cannot know, and thus the extent to which my model can be tested through this approach is questionable (Introducción; Litvin y Smith 2016).

(71) Second, the study used a random sampling technique for different types of nature-based tourism destination, which is a rigorous approach. However, the data are cross-sectional and may not be generalized to long-term ecotourism behavior (Unanue et al. 2016) (Conclusión; Lee y Jan 2018).

(72) Regarding our case studies, we cannot consider the branding strategies uncritically because, as Silva (2013) says, the making of heritage may give rise to two opposing impacts simultaneously- increased social cohesion and place pride, on the one hand, and envy and competition (and, thus, social atomisation), on the other hand- and residents are totally cognizant of the tension between the two. p. 14)... Branding is a form of essentialising, unifying and including part of a population under the umbrella of cultural identity and simultaneously; it is also a form of 
excluding those who cannot be protected under that umbrella (Conclusión; Jiménez-Esquinas y Sánchez-Carretero 2018).

(73) Local norms cover everything from the grammar and vocabulary of the language in use, to what is considered to be appropriate attire, matters of taste in cuisine, music, and humor; to laws proscribing who one may and may not marry; and to far from universal prohibitions against heinous crimes (Introducción; MacCannell 2016).

Las formas modales usadas son can y may. En todos los casos de can en los ejemplos (69), (70), (71) y (72), el significado es dinámico, pues parece no darse las condiciones o las circunstancias para que se puedan realizar las acciones descritas en cada ocasión: it cannot be denied..., we cannot know..., the data may not be generalized... y those who cannot be protected... Por otra parte, la perífrasis modal may not en el ejemplo (73), laws proscribing who one may and may not marry, la fuerza deóntica es externa. Esta fuerza es, en este caso, de orden legal, por lo que la realización solo puede ser llevada cuando no exista dicha fuerza deóntica. En este sentido, la posibilidad de casarse no depende de fenómenos aleatorios que pueden cumplirse en el futuro, esa posibilidad depende de la aplicación de una ley o normativa que decide con qué persona se puede casar un/a ciudadano/a del país que se describe en el artículo.

\subsubsection{Formular recomendaciones para investigadores}

Los siguientes ejemplos del corpus con should y must ilustran el uso de las perífrasis modales para presentar recomendaciones a colegas del ámbito académico: 
(74) ...tourism researchers, those most likely to analyze the theory, should applaud the model for what it is and not criticize it for what it is not (Introducción; Litvin y Smith 2016).

(75) Therefore, tourism scholarship seriously should consider new forms of protest, the affected areas and different forms in which cultural and social manifestations act strategically in urban areas that are experiencing abrupt changes, both symbolically and materially (Introducción; Bruttomesso 2018).

(76) Performing arts managers must refine the service concept, the service marketing direction and the market positioning strategy as being essential components of the service product strategy for the performing arts (Conclusión; Song 2016).

En el ejemplo (74) extraído de una introducción, los autores muestran su postura con respecto a un aspecto metodológico de su investigación. A diferencia de lo que sucede con la modalidad epistémica en la que los autores tratan de mitigar el posicionamiento, el uso de should en este caso particular busca un efecto que evidencie su compromiso con su observación final, a saber, que todas las fuentes de conocimiento disponibles requieren análisis. Jaime y Pérez-Guillot (2015: 294) encontraron que el modal should, similar al verbo modal must, es bastante común en el inglés técnico, en contraposición al inglés general, para expresar obligación y necesidad, y este significado ha sido atestiguado en nuestro corpus y se evidencia en las muestras presentadas aquí.

En el caso de should en (75), el valor deóntico indica una recomendación para que se lleve a cabo una mejora en aspectos de investigación. El ejemplo (76) ofrece un caso de 
must en una conclusión. Los autores explican la necesidad de que se lleve a cabo la mejora del concepto de servicio, de la dirección de marketing del servicio y de la estrategia de posicionamiento en el mercado se lleve a cabo para mejorar la situación descrita en el texto. Para Charlow (2016: 47), como se mencionó anteriormente, el uso de modales deónticos que indican necesidad justifica los significados deónticos con un sentido de necesidad en la idea de mejora o "betterness", usando su terminología. Desde una perspectiva formal, esto puede representarse de la siguiente manera:

P es mejor que $\neg \mathrm{P}$ si P permite

P es mejor que $\neg$ P si P evita la ambigüedad en la declaración

El significado más evidente de estas formas modales es el de obtención de un beneficio en el sentido de que las conclusiones que introducen estas formas modales se presentan como recomendaciones que deberían ser emprendidas por la industria turística o los gobiernos locales para mejorar la situación actual y, por consiguiente, ser más pertinentes desde el punto de vista económico, como ilustran los ejemplos analizados.

En todos estos casos, las perífrasis modales should y must indican la conveniencia de las acciones descritas en el contenido proposicional de las declaraciones en las que aparecen estos verbos. La idea de mejora sigue siendo fuerte en el uso de estas formas modales en muchos ejemplos con significado deóntico, especialmente en las conclusiones. El ejemplo (77) incluye unas pocas líneas de concordancia que contienen la expresión future research seguido de una perífrasis modal. 
(77) ...the study. Future research can corroborate (Peter y Anandkumar 2016).

...the data. Similarly, future research could adopt... (So et al. 2018).

...its findings. Therefore, future research may encompass tourists... (Correia et al. 2018).

... environment; so future research might aim to supplement... (Fitzgerald y Reis 2016).

...classification. Future research should also focus on... (Zaidan 2016).

Estas líneas de concordancia presentan los modales can, could, may, might y should que se combinan con verbos relacionados con procesos de investigación, como corroborate, aim, adopt, encompass, y supplement en estos ejemplos. Otros casos en el corpus incluyen las formas look at, address, adopt, test, employ, extend, focus on, aim to, attempt to, examine, investigate y validate, que corroboran esta asociación. Esta fórmula aparece con frecuencia en las conclusiones y, si bien puede parecer que indica cierto grado de autoridad, la intención es fundamentalmente manifestar líneas futuras de investigación, lo que suele ser algo establecido en esta y en otras disciplinas sobre todo en la sección dedicada a la conclusión.

\subsubsection{Formular recomendaciones para el sector turístico}

La función de formular recomendaciones para el sector turístico es, en esencia, igual que la descrita en la sección anterior. Sin embargo, la relevancia que tiene esta función en los artículos académicos en turismo determina que tenga una sección aparte. De hecho, el sentido utilitario de estos trabajos para colaborar con el tejido productivo en este sector es patente en todas las conclusiones analizadas en este trabajo. Los siguientes ejemplos son una evidencia de esto: 

IPO strategy by raising barriers to competition in order to strengthen their branding value and align their firm organization culture (Conclusión; Huang y Chang 2018).

(79) In addition to competition, a possible reason for low reciprocity is that most high-degree central websites are public organizations and information services that link many other businesses as a way of promoting and introducing them, whereas small businesses do not often link back to those public and information services. Thus, the highly central websites should consider requesting those small businesses they link to, to link back to them (Conclusión; Raisi et al. 2018).

Como se puede comprobar, el ejemplo (78) presenta la perífrasis modal must que introduce el consejo de mejora y, posteriormente, se incluye la consecuencia de esto con la estructura in order to. Se trataría en este caso de mejorar una estrategia de actuación con la que se potenciaría la imagen de la marca. De la misma manera, en el ejemplo (79), la forma modal should encuadra la recomendación consider requesting those small business they link to. La consecuencia se resuelve con el infinitivo de propósito to link back to them. Este uso de marcadores indicando propósito no es común, sin embargo, en la función de formular recomendaciones para los investigadores.

Juntamente con esta función de hacer recomendaciones, se aprecia en algunos ejemplos una función que persigue crear textura, como se aprecia en el siguiente ejemplo con la perífrasis modal should: 
(80) The findings in this study allow any pertinent recommendations for management of spa hotel. First, in today's world of intense competition in spa hotel industry, management and advertising objective should focus on both individual rationality and efficiency in increasing customer net value by improving overall perceptions of product and service quality with the setting of quality standards, for example, and/or by providing customers preferential treatment in price, as another. Second, male customers prefer higher quality of service compared to females; therefore promotion for both genders should be managed with different quality settings. Third, customers, who live in the urban areas. e.g. Northern and Central Taiwan), are more sensitive to the salient value of quality and price, however, are more eligible for promotional campaigns compared to the ones who live in the rural areas e.g. Southern and Eastern Taiwan). Finally, customers with more consumptive experience tend to have a higher willingness to pay for spa service than ones with fewer experiences, but the WTB/net value ratio tends to decrease when the consumptive frequency increases; therefore, the loyalty programme should be managed to improve customer retention (Conclusión; Shiu 2018).

En este ejemplo (80), se crea textura mediante la repetición de la misma forma modal should. Se ha marcado esta relación en el texto con cajas y líneas de unión. Se trata de un ejemplo donde persigue relacionar los hallazgos del estudio que se ha llevado a cabo en el artículo científico. Así, el uso de should para expresar consejos a partir de la 
investigación se refuerzan mediante el uso de marcadores textuales como first, second, therefore, y finally. Igualmente, se usa las perífrasis modales con esta misma función en (81):

(81) Up till now, they have made a modest use of the Internet in the tourism sector: $25 \%$ of hotels are present online and $10 \%$ of the restaurants, $50 \%$ of travel agencies, and $23 \%$ of car rental agencies have a Web site. They should provide an enjoyable Web site with easy to use navigation while providing the functionality needed to book online. They should also include space for the exchange of opinions and increase users' confidence through explicit safety measures particularly in light of this sector's relatively low take-up of online market- ing. Following the leads indicated in this study should help Web site designers increase the viewers' intention to book online... Tourism companies must first increase direct sales, through their Web sites, and also develop partnerships with online travel agents. In the meantime, tourism authorities should keep on investigating consumers' habits, and their perception of the Internet and of all technologies, as an e-commerce developing country such as Tunisia will move very quickly from traditional sales and distribution networks, to e- and m-distribution. The T-WAM, and future improved model and tools, adapted to the new social and technological context, contribute to a better understanding of travelers booking habits (Conclusión; Yousaf y Samreen 2016). 
En este ejemplo, es posible que se origine textura mediante la combinación de varias perífrasis modales que presentan una misma función. En este caso, las formas incluyen should, must y will, que presentan recomendaciones que son necesarias para la mejora del sector: they should provide an enjoyable Web site..., they should also include space for..., Following the leads in this study should help Web site designers..., Tourism companies must first increase direct sales..., tourism authorities should keep on investigating... La última perífrasis modal enmarca la conclusión que se desprendería del contexto de mejora descrito: an e-commerce developing country such as Tunisia will move very quickly from traditional sales and distribution networks, to e-and m-distribution. Se desprende, por lo tanto, de estos dos ejemplos el valor de las perífrasis modales para organizar la información que se presenta en el párrafo.

\subsection{Conclusiones}

Este capítulo se ha centrado en el estudio de las perífrasis modales en las introducciones y en las conclusiones de los artículos de investigación en turismo que pretende ser una contribución a los estudios de la modalidad, en general, y de las perífrasis modales en lengua inglesa, en particular, en el campo del discurso especializado. Por este motivo, los análisis se han centrado exclusivamente en el material extraído de revistas científicas en turismo que se han seleccionado siguiendo criterios basados en la calidad y la visibilidad de la investigación. Sin que este haya sido un objetivo trazado para este trabajo, se ha comparado los datos obtenidos en este estudio con los aportados por Alonso-Almeida y Carrió-Pastor (2017) en artículos científicos de lingüística e ingeniería. Además, de acuerdo con el objetivo principal de esta tesis, se ha identificado variación en el uso de los verbos modales en las secciones de los artículos de investigación sobre turismo examinadas aquí. 
La primera conclusión de este estudio está relacionada con las formas que se han detectado en cada una de las secciones. Así, las formas más frecuentes que aparecen en las conclusiones, según las frecuencias relativas, son can, will y may, por este orden. En las conclusiones, las frecuencias relativas de formas modales son mayores que en las introducciones. Las formas más comunes son can, may, could y should. En la mayoría de los casos, existe variación significativa en el uso de cada una de las perífrasis en las dos secciones. Sin duda, la forma que presenta un efecto estadísticamente grande es should con un uso muy marcado en las conclusiones.

Otra conclusión relevante es que la modalidad epistémica aparece con mayor frecuencia en las conclusiones, siendo segunda en las introducciones, mientras que la modalidad dinámica es más frecuente en las introducciones. Este valor dinámico es, sin embargo, el tercero más común en las conclusiones. En el caso de la modalidad deóntica, es más común en las conclusiones que en las introducciones. Los mecanismos evidenciales presentan mayor frecuencia relativa las conclusiones que en las introducciones, probablemente debido a que, en esta sección, pueden aparecer razonamientos para justificar las conclusiones que se aportan.

En cuanto a las funciones, se han detectado varias que estas perífrasis cumplen en los textos. Estas funciones están estrechamente relacionadas con los significados modales. Así, la función de mitigación y cortesía lingüística se relaciona en mayor medida con la modalidad epistémica y lo mismo ocurre con la formulación del significado inferencial, que refiere a la necesidad epistémica. En otras palabras, la modalidad epistémica puede usarse para mostrar el análisis lógico que subyace en la argumentación de los autores. En general, la pragmática de este tipo de modalidad en las secciones analizadas es la 
manifestación de una falta de compromiso con la información presentada con el fin de evitar la imposición.

Otras funciones de las perífrasis modales que se han detectado en las introducciones y las conclusiones son la expresión de la factualidad y la de la imposibilidad de realizar una acción; estas dos suelen vincularse con la modalidad dinámica. En el caso de la modalidad deóntica, esta suele aparecer relacionada con las funciones predictiva, la indicación de la organización, la expresión de la autoridad y la formulación de recomendaciones a autores y las dirigidas al sector turístico. En ocasiones, se ha mostrado la manera en la que el empleo de las formas modales se combina con otros elementos textuales para reforzar la intención comunicativa de los autores.

Además de todas estas funciones y sin realmente estar asociada a ningún tipo de modalidad, se ha constatado el empleo de formas modales en un párrafo con la función de crear textura. De esta manera, se consigue estructurar el contenido de un texto al mismo tiempo que se presenta la perspectiva de los autores. El uso de varias formas modales puede darse repitiendo la misma forma en el desarrollo de una idea o formas diferentes, pero cuya función es la misma en el párrafo. 



\section{Conclusiones}

Esta tesis tenía como objetivo principal el análisis de las perífrasis modales, sus significados en las introducciones y las conclusiones en los artículos de investigación en la disciplina de turismo, además de detectar la variación existente en el uso de estas formas verbales entre estas secciones. En este sentido, han sido tres las preguntas que se formularon al comienzo de esta investigación y que se responderán de manera resumida en esta sección, de acuerdo con los análisis hechos y expuestos en este trabajo. Estas cuestiones se usan para organizar la información en este capítulo.

Para poder analizar las perífrasis modales, se ha presentado la definición y los tipos modales que guiaría la clasificación de las formas encontradas en los textos. Así, Palmer (1990, 2001) aparece como una fuente de referencia y, de ahí, esta tesis toma las categorías modales epistémica y evidencial, deóntica y dinámica. De la misma manera, se ha definido lo que es una perífrasis modal central, siguiendo los criterios recogidos en Denison (1993). Junto con esto, se definió siguiendo el modelo de estructura genérica recogido en Alonso-Almeida y Álvarez-Gil (2021a), lo que ha permitido caracterizar los textos usados para formar el corpus de trabajo mediante la identificación previa de las introducciones y las conclusiones usando también las fórmulas léxico-gramaticales que aparecen de manera recurrente en estas secciones. 


\subsection{Primera cuestión: Las formas de las perífrasis modales}

La primera cuestión propuesta buscaba saber qué formas de perífrasis modales son las que aparecen con mayor frecuencia en estas secciones del artículo académico. En este sentido, un análisis global de estas formas modales señaló la existencia de variación entre su frecuencia de uso en las introducciones y las conclusiones. De manera detalla, can es la forma más usada en el corpus con una mayor presencia en las conclusiones con respecto a las introducciones. Esto ocurre también con la forma could. En el caso de may, se encontraron más casos en las conclusiones con un efecto grande en cuanto a la variación existente entre las secciones. Igualmente, might presenta mayor frecuencia en las conclusiones. En los casos de must, will, would y should, estas formas exhiben más casos en las conclusiones. La forma shall es más común en las introducciones, aunque el efecto de variación es pequeño.

Una comparativa con los datos extraídos de artículos de lingüística y de ingeniería muestra las diferencias y las similitudes. La forma can presenta mayor frecuencia en la introducción en los Al de lingüística e ingeniería. Sin embargo, la frecuencia de uso de can en las conclusiones es mayor en los textos de ingeniería seguido del de turismo y de lingüística. En cuanto a could, esta forma es menos frecuente en las introducciones de textos de lingüística seguida del de ingeniería y el de turismo. En las conclusiones, la forma could muestra una frecuencia mayor en los trabajos de investigación en turismo y de 12,69 en los trabajos de investigación en lingüística. Por otra parte, no hay casos de could en los textos de ingeniería.

El modal may presenta menos casos en las introducciones de los trabajos de investigación en lingüística que en turismo e ingeniería. En las conclusiones, no existe coincidencia 
entre ninguno de los corpus, y esta paráfrasis modal tiene las frecuencias más altas en lingüística, seguida por las de turismo normalizadas y, finalmente, las de ingeniería. El verbo might puede aparecer con más frecuencia en las introducciones de ingeniería y con menos frecuencia en las introducciones de turismo. La diferencia está en las conclusiones, con las frecuencias normalizadas más bajas en la disciplina de turismo y sin casos en ingeniería. El verbo must aparece con más frecuencia en los textos de ingeniería, seguido de los textos de turismo y de lingüística, en este orden. En la conclusión, esta forma está presente en todos los corpus comparados, con mayor frecuencia en los artículos de investigación de lingüística y turismo. La forma shall aparece más a menudo en las introducciones y en las conclusiones de los artículos en turismo, mientras que no se detectaron casos en los otros corpus. En cuanto a should, aparece solo en las introducciones de turismo. En las conclusiones de estos artículos, should tiene una frecuencia mayor, seguido por el de ingeniería) y el de lingüística.

Se comprobó la existencia de diferencia en la frecuencia de uso de will en todos los corpus comparados. Aparece con mayor frecuencia en los textos de lingüística, seguido por el de ingeniería y turismo. En las conclusiones, will aparece más a menudo en el corpus de textos de turismo y con una frecuencia menor en el corpus de ingeniería y en el corpus de lingüística, por este orden. Por último, would tiene frecuencias parecidas en las introducciones en los tres corpus mientras que la frecuencia es mayor en las conclusiones del corpus de lingüística mientras que el corpus de turismo e ingeniería presentan menos casos de esta forma modal, en este orden. 


\subsection{Segunda cuestión: Los significados de las perífrasis modales}

La segunda cuestión plantea qué significados modales son más frecuentes en la introducción y en la conclusión y si la variación existente es significativa. La modalidad dinámica es el significado modal preferido en las introducciones, mientras que la modalidad epistémica aparece con mayor frecuencia en las conclusiones. La modalidad epistémica y la modalidad deóntica, en este orden, son las siguientes más comunes en las introducciones. La modalidad deóntica se usa como segundo recurso modal en las conclusiones seguido del dinámico. Los mecanismos evidenciales aparecen en las dos secciones con una frecuencia relativa superior en el caso de los textos en las conclusiones

Tras el análisis de los datos, se han comparado los resultados de las perífrasis modales encontradas en los análisis realizados con los de Alonso-Almeida y Carrió-Pastor (2017). Así, se revela que la modalidad dinámica es el significado modal preferido en las introducciones de todos los corpus examinados, pero no hay mucha diferencia entre los significados epistémicos y dinámicos en el caso del corpus de ingeniería. La modalidad epistémica se da con mayor frecuencia en las introducciones de los textos de ingeniería y es menos frecuente en el corpus de turismo y de lingüística, respectivamente. La modalidad deóntica es más frecuente en las introducciones de lingüística y turismo, en este orden, y con una frecuencia ligeramente menor en las introducciones de turismo. En cuanto a las conclusiones, el significado modal más habitual es el epistémico en el caso de los textos de lingüística y de turismo. El dinámico es el siguiente significado modal más frecuente en estas conclusiones en ingeniería y lingüística. En el caso del significado deóntico, este es el segundo modal más usado en el corpus de turismo. Los valores evidenciales solo se hallaron en los textos de turismo, con mayor presencia en las conclusiones. 
Las perífrasis modales epistémicas en el corpus analizado son may, would, could, y might en las introducciones y las conclusiones, en este orden de frecuencia. En todas las perífrasis modales epistémicas, se identificó un valor evaluativo con una intención de proporcionar interpretaciones provisionales de los fenómenos analizados en el artículo, en el caso de las conclusiones, con un valor atenuante del contenido proposicional. Se constató que existe variación en el caso de todas las formas epistémicas de acuerdo con su presencia en la introducción y en la conclusión, con mayor frecuencia en las conclusiones. La forma could presenta la mayor probabilidad de que se produzca esta forma con significado epistémico en la conclusión. En cuanto la modalidad evidencial, las formas que se usan con este valor en nuestro corpus son could, may, might, would y should. La forma que presenta mayor variación es may con una mayor probabilidad de que aparezca en la conclusión que en la introducción. En el caso de might se comprobó que hay mayor probabilidad de frecuencia de aparición de esta perífrasis en la introducción que en la conclusión.

Las perífrasis modales dinámicas utilizadas en las introducciones de los textos son can, will, could, may, will y would en este orden de frecuencia. Esta forma aparece con una frecuencia relativa mayor en el caso de las conclusiones. El uso de can se refiere a la posibilidad dinámica en cuanto a la realización de la acción descrita en la proposición basándose en las características que son específicas de las personas, objetos y eventos referidos. En términos generales, la modalidad dinámica es más frecuente en las conclusiones de los artículos de investigación en turismo con mayor probabilidad de que aparezcan las formas can, could y may en las conclusiones. Este uso se debe a que estas estructuras favorecen la presentación de ideas evitando la imposición de la perspectiva de los autores. 
La modalidad deóntica es la menos frecuente de los tres tipos de modalidad en las introducciones y las conclusiones de los artículos de investigación. La modalidad deóntica se expresa por medio de las formas modales can, must, will, shall y should, en las introducciones, y lo mismo ocurre en las conclusiones, además de could. Las nociones deónticas que se han constatado son la necesidad, la predicción y la expectativa. Siguiendo a Charlow (2016), se comprueba que, en las conclusiones, el uso de este modal se fundamenta en la idea de "mejoría" que subyace en la expresión de la necesidad deóntica de llevar a cabo una acción que, como consecuencia, ofrezca beneficios al sector turístico. Se aprecia en los análisis realizados que could, must, will y should presentan mayor probabilidad de presentar variación en las conclusiones que en las introducciones.

\subsection{Tercera cuestión: Las funciones de las perífrasis modales}

La tercera, y última, cuestión gira en torno a qué funciones cumplen estas perífrasis modales en los textos. En principio, la función de las perífrasis modales es expresar la perspectiva del hablante con respecto al contenido proposicional, lo que implica una evaluación del contenido proposicional. Sin embargo, existen una serie de funciones específicas de las perífrasis modales que justifican su empleo por los hablantes. En este trabajo, se han identificado las siguientes funciones en el corpus de introducciones y conclusiones de artículos científicos analizados en esta tesis, a saber: mitigación y cortesía lingüística, predicción, factualidad, significado inferencial, indicación de la organización del texto, autoridad, imposibilidad de realizar una acción, recomendaciones tanto a colegas académicos y a empresas y organizaciones públicas en el sector turístico.

En el corpus, la mitigación se lleva a cabo principalmente mediante estructuras epistémicas, Esta función pretende evitar tanto la imposición del punto de vista, como la 
confrontación por cuestiones de discrepancias. Además, el uso de estas estrategias implica la falta de compromiso de los autores con la proposición expresada, lo que, en términos de cortesía, se clasificarían como estrategias de cortesía negativa para evitar imponer la perspectiva de los hablantes, ya que se indicaría duda o ausencia de certeza. En cuanto a la función de predicción en las introducciones y en las conclusiones, se usan perífrasis modales con significado deóntico para este fin. Se observó que algunas formas modales combinan con elementos léxicos y otras estructuras morfológicas para indicar un razonamiento lógico en la elaboración del conocimiento. La expresión de la factualidad en el corpus se expresa mediante el uso de perífrasis modales dinámicas en las conclusiones para reforzar el punto de vista en la comunicación, pues este significado modal hace no hace referencia a una posibilidad aleatoria, sino a una posibilidad basada en la presunción de que una persona, un evento o un objeto tiene la capacidad intrínseca o en las condiciones externas para que realicen la acción descrita en la proposición. Con todo, estas perífrasis pueden mitigar el contenido proposicional, al expresar hechos potencialmente posibles y/o habilidades con lo que la perspectiva del hablante está basada en una percepción objetiva de la realidad.

En cuanto al uso de las perífrasis modales con significado inferencial, se usan como mecanismos de estrategia de cortesía negativa para evitar la imposición a los destinatarios del mensaje mediante la expresión del razonamiento deductivo usado por los autores y las autoras en la elaboración de nuevo conocimiento. Se revela, por lo tanto, la naturaleza cognitiva del procedimiento lo que mitiga, además, la fuerza elocutiva de la proposición al presentar el hecho como un elemento subjetivo.

Especialmente en las introducciones, las perífrasis modales deónticas indican la organización del artículo de investigación, pues el empleo de estas formas manifiesta la 
intención de los autores en el diseño de su propio trabajo. Esta función se apoya, además, en otros marcadores adverbiales y elementos léxicos, como se ha mostrado en este trabajo. La modalidad deóntica también se usa para expresar autoridad. Igual que se puede mitigar el contenido proposicional para evitar críticas posteriores ante una aseveración directa es posible mostrar el grado de implicación como especialista de los autores para garantizar la fiabilidad de la información que se ofrece. De otra manera, la información parecería carecer de validez por exceso de atenuación que impida comprobar la implicación de sus autores con la veracidad de la información que se desprende de la investigación que se haya realizado.

Otra función que se ha identificado en este corpus es la expresar la imposibilidad de llevar a cabo la acción que se describe en la proposición. Por lo general, esta imposibilidad se manifiesta en la incapacidad o la ausencia de condiciones que promuevan la realización de dicha acción. Este uso se encuadraría como un mecanismo de cortesía negativa para evitar la imposición del punto de vista, pues la conclusión refleja un hecho objetivo basado en las propias cualidades del sujeto, o bien en los condicionantes que lo permitan.

Se ha constatado igualmente que la recomendación es una función clave en los textos turísticos, pues no solo se dirige a colegas del ámbito académico, sino a empresas y organizaciones del sector turístico, con el significado que tiene para el avance del sector. Estas recomendaciones se formulan usando perífrasis modales deónticas, preferiblemente should, en el corpus analizado. Así, los autores explican la necesidad de que se lleve a cabo una mejora del ámbito académico o del ámbito profesional en la industria turística. Esta idea de mejora es notoria en el uso de estas formas modales, sobre todo en las conclusiones. Esto es fundamentalmente igual en el caso de las recomendaciones destinadas al sector turístico. Este tipo de función de la modalidad se 
basa en el sentido utilitario de los artículos de investigación para colaborar con el tejido productivo en este sector, por lo que no es extraño que se produzca en las conclusiones de estos trabajos. La perífrasis con esta función puede ir seguidas de estructuras que indiquen la razón o consecuencia de realizar la recomendación expresada.

Finalmente, se observó de manera tangencial que la repetición de una forma modal o el uso de varias en un mismo texto puede contribuir a la creación de textura, lo que redunda, por una parte, en una organización meditada de los contenidos y, por otra, permite reforzar el posicionamiento de los autores del artículo científico en la investigación realizada.

\subsection{Investigación futura}

Esta tesis ha demostrado que existe variación en cuanto a las formas y a los significados modales que presentan las perífrasis modales en las introducciones y las conclusiones de los artículos de investigación en turismo. De la misma manera, se ha confirmado que estas formas modales cumplen una serie de funciones específicas que contribuyen a la elaboración del conocimiento científico. Sin embargo, este estudio solo representa una aproximación a la caracterización textual del artículo científico que permita extraer conclusiones con fines didácticos tanto para especialistas en el ámbito turístico como para estudiantes en esta disciplina.

A pesar del estudio planteado por Alonso-Almeida y Álvarez-Gil (2021a), es necesario emprender más trabajos como este que permita conocer con más detalles la estructura genérica de los artículos de investigación en un corpus grande de estos textos. Esto permitirá, a su vez, poder acometer nuevos análisis de las perífrasis modales en todas las 
secciones del artículo en turismo para conocer con exactitud estos usos y la razón de su empleo.

En este estudio, se ha observado que la modalidad interactúa con otros marcadores textuales para reforzar un determinado aspecto de la investigación. De aquí, se desprende la necesidad de llevar trabajos sobre elementos textuales y de interacción (en el sentido de Hyland (2005b)) que, en su totalidad, den una imagen tanto de las funciones que cumplen como de la manera en la que se relacionan, así como la frecuencia en que esto ocurre. Estos datos pueden ser comparados con los datos obtenidos para otras disciplinas para averiguar la variación que existe en cada caso.

Igualmente, sería muy interesante comprobar la relación existente entre las formas perifrásticas modales y la expresión de la cortesía. Se ha visto en esta tesis que, si bien parece existir cierta correlación en el uso de las perífrasis modales epistémicas y la mitigación lingüística como mecanismo de cortesía para evitar la imposición del punto de vista, esto no parece ser exclusivo de esta categoría modal, pues otros significados, como el dinámico, parece igualmente adecuado para indicar esta función, como señala AlonsoAlmeida (2021). Esto se podría llevar a cabo en un corpus de artículos científicos en el registro turístico.

Finalmente, sería también muy interesante comprobar si realmente existe una recepción real mediante transferencia de las contribuciones de los artículos académicos en el sector turístico para poder saber si esto se hace de manera directa a través de estos artículos o por medio de otros géneros textuales, como puede ser el informe. La presunción de la lectura de estos textos por parte de las personas interesadas del sector turístico justificaría la expresión clara y directa de las recomendaciones. En otras palabras, las 
recomendaciones en las conclusiones se expresan solo mediante el uso de perífrasis modales deónticas con una elaboración textual causa-efecto sin otros adornos estilísticos que puedan oscurecer el significado. Así, se validaría la idea manifestada en el análisis de las funciones sobre la importancia que tiene la modalidad deóntica, si bien es la menos usada de las tres, en las conclusiones de los trabajos. 

Bibliografía 



\section{Bibliografía}

Ahmed, Shabbir. (2015). Rhetorical Organization of Tourism Research Article Abstracts. Procedia - Social and Behavioral Sciences,vol.208, 269 - 281. https://doi.org/10.1016/i.sbspro.2015.11.203

Aikhenvald, Alexandra. (2004). Evidentiality. Oxford: Oxford University Press.

Allott, Nicholas. (2010). Key Terms in Pragmatics. Nueva York: Continuum.

Almeida, Sandra \& Lilian Ferrari. (2010). Subjectivity, Intersubjectivity and Epistemic Complementation Constructions. En Christopher Hart (Ed.), Selected Papers from the 3rd UK Cognitive Linguistics Conference. Volume I, 110-127. http://www.ukcla.org.uk/files/proceedings/Almeida \%0Dand Ferrari.pdf [Acceso: 10/05/2020].

Alonso-Almeida, Francisco. (2008). The Middle English medical charm: Register, genre and text type variables. Neuphilologische Mitteilungen, vol. 109(1), 9-38. http://www.jstor.org/stable/43344333

Alonso-Almeida, Francisco. (2014). Evidential and epistemic devices in English and Spanish medical, computing and legal scientific abstracts: A contrastive study. Linguistic Insights, vol. 187, 21-42. Bern, Berlin, New York: Peter Lang.

Alonso-Almeida, Francisco. (2015a). On the mitigating function of modality and evidentiality. Evidence from English and Spanish medical research papers. Intercultural Pragmatics, vol. 12(1), 33-57. https://doi.org/10.1515/ip-2015-0002

Alonso-Almeida, Francisco. (2015b). Introduction to stance language. Research in Corpus Linguistics, vol. 3, 1-5. 
Alonso-Almeida, Francisco. (2015c). The functions of seem and parecer in early medical writing. Discourse Studies, vol. 17(2), 121-140. https://doi:10.1177/1461445614564517

Alonso-Almeida, Francisco. (2015d). Sentential epistemic and evidential devices in Spanish and English texts on computing. En Juan Rafael Zamorano-Mansilla, Carmen Maíz, Elena Domínguez \& María Victoria Martín de la Rosa (Eds.), Thinking Modally: English and Contrastive Studies on Modality, 383-408. Newcastle upon Tyne: Cambridge Scholars Publishing.

Alonso-Almeida, Francisco. (2021). Los modales dinámicos en textos de historia en lengua inglesa (1700-1900). Signos. Revista de lingüística, vol. 54(106), 529-548. https://10.4067/S0718-09342021000200529

Alonso-Almeida, Francisco \& Francisco J. Álvarez-Gil (2019). Modal verb categories in CHET. En Isabel Moskowich, Begoña Crespo, Luis Puente-Castelo \& Leida Maria Monaco (Eds.), Writing History in Late Modern English: Explorations of the Coruña Corpus, 150-165. Amsterdam: John Benjamins Publishing Company. https://doi.org/10.1075/2.225.08alo

Alonso-Almeida, Francisco \& Francisco J. Álvarez-Gil. (2020). 'so that it may reach to the jugular'. Modal Verbs in Early Modern English Recipes. Studia Neofilologiczne, vol. XVI, 61-88. https://doi.org/10.16926/sn.2020.16.04

Alonso-Almeida, Francisco \& Francisco J. Álvarez-Gil. (2021a, en prensa). The macrostructure of tourism research articles and associated language features. JEAP.

Alonso-Almeida, Francisco \& Francisco J. Álvarez-Gil. (2021b, en prensa). Developing Argumentation in History Texts: Epistemic Modality and Evidentiality. Pragmalingüística, vol. 29.

Alonso-Almeida, Francisco \& María Luisa Carrió-Pastor. (2016). Evidentiality and Modality in European Languages. En Juana I. Marín-Arrese, Julia Lavid-López, Marta Carretero, Elena Domínguez Romero, M ${ }^{a}$ Victoria Martín de la Rosa \& María Pérez Blanco (Eds.), Evidentiality and Modality in European Languages: Discoursepragmatic perspectives, 187-208. Bern: Peter Lang.

Alonso-Almeida, Francisco \& María Luisa Carrió-Pastor. (2017). Variation and Function of Modals in Linguistics and Engineering Research Papers in English. En Juana I Marín- 
Arrese, Julia Lavid-López, Marta Carretero, Elena Domínguez Martín de la Romero, $M^{a}$ Victoria Rosa \& María Pérez Blanco (Eds.), Evidentiality and Modality in European Languages. Discourse- pragmatic perspectives, 277-311. Bern: Peter Lang.

Alonso-Cortés, Ángel. (2008). Lingüística. 2nd ed. Madrid: Cátedra Ediciones.

Aluthman, Ebtisam Saleh. (2018). A Cross-disciplinary Investigation of Textual Metadiscourse Markers in Academic Writing. International Journal of Linguistics, vol. 9(6), 164. https://doi.org/10.5296/ijl.v9i6.12310

Álvarez-Gil, Francisco J. (2018). Epistemic modals in early Modern English history texts. Analysis of gender variation. Revista de Lingüística y Lenguas Aplicadas, vol. 13(1), 13-20. https://doi.org/10.4995/rlyla.2018.7801

Álvarez-Gil, Francisco J. (2021). Authority and deontic modals in Late Modern English. In Isabel Moskowich, Inés Lareo \& Gonzalo Rioboo-Camiña (Eds.), "All families and genera". Exploring the Corpus of English Life Sciences Texts, 249-264. Amsterdam: John Benjamins Publishing Company.

Álvarez-Gil, Francisco J. \& Elena Domínguez-Morales. (2018). Modal verbs in the abstract genre in the field of tourism. Revista de lenguas para fines especificos, vol. 24(2), 27-37. https://doi.org/10.20420/rlfe.2018.232

Álvarez-Gil, Francisco J., Payet, Karine Marie Muriel, \& Ángeles Sánchez-Hernández (2020). Analyse de la modalité dans quelques textes touristiques aux Îles Canaries. Studii şi cercetări filologice. Seria Limbi străine aplícate, vol. 19, 9-20.

Álvarez-Gil, Francisco J. \& Marina Bondi (2021). Introduction to the monographic section: Metadiscourse devices in academic discourse. Revista Signos. Estudios de Lingüística, vol. 54(106), 518-528.

Álvarez-Gil, Francisco J. \& María Elena Domínguez-Morales. (2021). Modal verbs in academic papers in the field of tourism. Revista Signos. Estudios de Lingüística, vol. 54(106), 549-574. https://10.4067/S0718-09342021000200549

Aquino, Richard S., Heike A. Schänzel \& Kenneth F. Hyde. (2018). Unearthing the geotourism experience: Geotourist perspectives at Mount Pinatubo, Philippines. Tourist Studies, vol. 18(1), 41-62. https://doi.org/10.1177/1468797617717465

Auwera, Johan Van der \& Vladimir A. Plungian. (1998). Modality's semantic map. 
Linguistic Typology, vol. 2(1), 79-124. https://doi.org/10.1515/lity.1998.2.1.79

Azar, Ali Sorayyaei. (2012). The Self-Promotion of Academic Textbooks in the Preface Section: A Genre Analysis. Atlantis, vol. 34(2), 147-165.

Beijering, Karin. (2017). Grammaticalization and (inter)subjectification: The case of the Swedish modals må and matte. En Daniël Van Olmen, Hubert Cuyckens \& Lobke Ghesquière (Eds.), Aspects of Grammaticalization (Inter)Subjectification and Directionality, 47-80. Berlin/Boston: Walter de Gruyter GmbH. https://doi.org/10.1515/9783110492347-003

Benkraiem, Ramzi, Amine Lahiani, Anthony Miloudi \& Muhammad Shahbaz. (2020). A new look at the tourism development and economic growth nexus: International evidence. Tourism Economics, 1-29. https://doi.org/10.1177/1354816620938482

Beritelli, Pietro \& Stephan Reinhold. (2018). Chance meetings, the destination paradox, and the social origins of travel: Predicting traveler's whereabouts? Tourist Studies, vol. 18(4), 417-441. http://doi:10.1177/1468797617748292

Bhatia, Vijay Kumar. (1993). Analysing Genre: Language Use in Professional Settings. Londres: Longman.

Biber, Douglas. (1988). Variation Across Speech and Writing. Cambridge: Cambridge University Press.

Biber, Douglas y Edward Finegan. (1989). Styles of stance in English: Lexical and grammatical marking of evidentiality and affect. Text, vol. 9(1), 93-124. http://doi:10.1515/text.1.1989.9.1.93.

Biber, Douglas, Stig Johansson, Geoffrey Leech, Susan Conrad \& Edward Finegan. (1999). Longman Grammar of Spoken and Written English. Londres: Longman.

Bolderman, Leonieke \& Stijn Reijnders. (2017). Have you found what you're looking for? Analysing tourist experiences of Wagner's Bayreuth, ABBA's Stockholm and U2's Dublin. Tourist Studies, vol. 17(2), 164-181. http://doi:10.1177/1468797616665757

Boye, Kasper \& Peter Harder. (2009). Evidentiality: Linguistic categories and grammaticalization. Functions of Language, vol. 16(1), 9-43. http://doi:10.1075/fol.16.1.03boy 
Brown, Lucien. (2011). Korean Honorifics and Politeness in Second Language Learning (Pragmatics \& Beyond New Series). Amsterdam: John Benjamins Publishing Company. http://doi:10.1075/pbns.206

Brown, Penelope \& Stephen Levinson. (1987). Politeness: Some Universals in Language Usage. Cambridge: Cambridge University Press.

Bybee, Joan L., Revere D. Perkins \& William Pagliuca. (1994). The evolution of grammar: tense, aspect, and modality in the languages of the world. Chicago: University of Chicago Press.

Caffi, Claudia. (1999). On mitigation. Journal of Pragmatics, vol. 31(7), 881-909. http://doi:10.1016/S0378-2166(98)00098-8

Caffi, Claudia. (2007). Mitigation (Studies in Pragmatics). Londres: Elsevier.

Carretero, Marta \& Juan Rafael Zamorano-Mansilla. (2015). Evidentiality as Conversational Implicature: Implications for Corpus Annotation. Procedia - Social and Behavioral Sciences, vol. 212, 146-150. http://doi:10.1016/i.sbspro.2015.11.312

Carrió-Pastor, María Luisa. (2012). A contrastive analysis of epistemic modality in scientific English. Revista de lenguas para fines específicos, vol. 18, 115-132. https://ojsspdc.ulpgc.es/ojs/index.php/LFE/article/view/39

Carrió-Pastor, María Luisa. (2013). A contrastive study of the variation of sentence connectors in academic English. Journal of English for Academic Purposes, vol. 12(3), 192-202. http://doi:10.1016/j.jeap.2013.04.002

Carrió-Pastor, María Luisa. (2014). Cross-cultural variation in the use of modal verbs in academic English. SKY Journal of Linguistics, vol. 27, 153-166.

Carrió-Pastor, María Luisa. (2016). Mitigation of claims in medical research papers: A comparative study of English and Spanish writers. Communication and Medicine, vol. 13(3), 249-261. http://doi:10.1558/cam.28424.

Carrió-Pastor, María Luisa. (2020a). Cross-cultural analysis of English modal constructions in engineering academic papers. Odisea: Revista de estudios ingleses, vol. 20, 75-90.

Carrió-Pastor, María Luisa. (2020b). Epistemic modals in academic English. En Pascal 
Hohaus \& Rainer Schulze (eds.), Re-Assessing Modalising Expressions: Categories, co-text, and context, 253-280. Amsterdam: John Benjamins.

Carrió-Pastor, María Luisa. (2020c). Conocer la lengua a través de los corpus: la herramienta METOOL, retos para el análisis de los marcadores discursivos. Pragmalingüística, vol. $28,255-274$.

Carrió-Pastor, María Luisa \& Rut Muñiz Calderón. (2015). A Contrastive Analysis of Metadiscourse Features in Business e-mails Written by Non-native Speakers of English. Procedia - Social and Behavioral Sciences, vol. 173, 214-221. http://doi:10.1016/i.sbspro.2015.02.055.

Carrió-Pastor, María Luisa \& Francesca Romero-Forteza. (2014). Second Language Writing: Use of the World Wide Web to Improve Specific Writing. Procedia - Social and Behavioral Sciences, vol. 116, 235-239. http://doi:10.1016/j.sbspro.2014.01.200.

Chafe, Wallace. (1986). Evidentiality in English conversation and academic writing. En William Chafe \& Johanna Nichols (eds.), Evidentiality: The linguistic coding of epistemology, 261-272. Norwood: Ablex.

Charlow, Nate \& Matthew Chrisman. (2016). Deontic Modality. Oxford: Oxford University Press.

Cheng, Winnie \& Le Cheng. (2014). Epistemic modality in court judgments: A corpusdriven comparison of civil cases in Hong Kong and Scotland. English for Specific Purposes, vol. 33, 15-26. http://doi:10.1016/i.esp.2013.07.006.

Chu, Christina, Mark Rosenfield, Joan K. Portello, Jaclyn A. Benzoni \& Juanita D. Collier. (2011). A comparison of symptoms after viewing text on a computer screen and hardcopy. Ophthalmic and Physiological Optics, vol. 31(1), 29-32. http://doi.wiley.com/10.1111/i.1475-1313.2010.00802.x.

Closs Traugott, Elizabeth. (2003). Approaching modality from the perspective of Relevance Theory. Language Sciences, vol. 25(6), 657-669. http://doi:10.1016/S0388-0001(03)00017-2.

Coates, Jennifer. (2003). The role of epistemic modality in women's talk. En Roberta Facchinetti, Manfred Krug \& Frank Palmer (Eds.), Modality in Contemporary English, 331-348. Berlin, Nueva York: Mouton de Gruyter. 


\section{https://doi.org/10.1515/9783110895339.331.}

Collins, Peter. (2009). Modals and Quasi-modals in English. Amsterdam, Nueva York: Rodopi.

Comrie, Bernard. (1985). Tense. Cambridge: Cambridge University Press. http://doi:10.1017/CBO9781139165815.

Cornillie, Bert. (2009). Evidentiality and epistemic modality: On the close relationship between two different categories. Functions of Language, vol. 16(1), 44-62. http://doi:10.1075/fol.16.1.04cor.

Cutting, Joan. (2007). Introduction to Vague language explored. En Joan Cutting (Ed.), Vague Language Explored, 3-17. Nueva York: Palgrave Macmillan.

Cuyckens, Hubert, Kristin Davidse \& Lieven Vandelanotte. (2010). Introduction. En Hubert Cuyckens, Kristin Davidse \& Lieven Vandelanotte (Eds.), Subjectification, Intersubjectification and Grammaticalization, 1-26. Berlin, New York: De Gruyter Mouton.

Czerwionka, Lori. (2012). Mitigation: The combined effects of imposition and certitude. Journal of Pragmatics vol 44(10), 1163-1182. http://dx.doi.org/10.1016/j.pragma.2012.05.002.

Denison, David. (1993). English Historical Syntax: Verbal Constructions (Longman Linguistics Library). Londres, Nueva York: Longman.

Depraetere, Ilse. (2017). On the pragmatics of modal verbs. Selected Papers on theoretical and applied linguistics, vol. 22, 14-26. https://doi.org/10.26262/istal.v22i0.5969.

Dikgang, Johane \& Edwin Muchapondwa. 2017. The determination of park fees in support of benefit sharing in Southern Africa. Tourism Economics, vol. 23(6), 1165-1183. https://doi:10.1177/1354816616655254.

Dixon, Robert Malcolm Ward. (2005). A Semantic Approach to English Grammar. Oxford: Oxford Universit Press.

Dolnicar, Sara \& Alexander Chapple. (2015). The readability of articles in tourism journals. Annals of Tourism Research, vol. 52, 161-166. http://doi:10.1016/j.annals.2015.03.003. 
Dumbrăveanu, Daniela, Duncan Light, Craig Young \& Anya Chapman. (2016). Exploring women's employment in tourism under state socialism: Experiences of tourism work in socialist Romania. Tourist Studies, vol. 16(2), 151-169. http://doi:10.1177/1468797615594747.

Efstathiadi, Lia. (2010). The use of epistemic markers as a means of hedging and boosting in the discourse of L1 and L2 speakers of Modern Greek: A corpus-based study in informal letter-writing. Themes in Science and Technology Education: Special issue on ICT in Language Learning. Computer-aided language analysis, teaching and learning: Approaches, perspectives and applications, vol. 3(1-2), 181-206. https://files.eric.ed.gov/fulltext/EJ1131486.pdf. [Acceso: 06/03/2020].

Eggins, Suzanne. (1994). An Introduction to Systemic Functional Linguistics. Londres: Pinter Publishers.

Facchinetti, Roberta, Manfred Krug \& Frank Palmer. (2003). Preface. En Roberta Facchinetti, Manfred Krug \& Frank Palmer (Eds.), Modality in Contemporary English. Topics in English Linguistics 44, v-xiii. Berlin, Nueva York: Mouton de Gruyter.

Fairclough, Norman. L. (2004). Analysing Discourse: Textual Analysis for Social Research. NuevaYork: Routledge.

Farsari, loanna. 2018. A structural approach to social representations of destination collaboration in Idre, Sweden. Annals of Tourism Research, vol. 71, 1-12. https://doi:10.1016/j.annals.2018.02.006.

Fintel, Kai von. (2006). Modality and Language. En D. M. Borchert (Ed.), Encyclopedia of Philosophy - Second Edition, 20-27. Detroit, MI: Macmillan.

Fitzgerald, Jon \& Arianne C. Reis. (2016). Island intersections: Music and tourism on Fernando de Noronha, Brazil. Tourist Studies, vol. 16(2), 170-191. http://doi:10.1177/1468797615594738.

Flores, Nydia. (2020). Linguistic mitigation in English and Spanish: How speakers attenuate expressions. Londres: Routledge.

Flowerdew, John. (2013). Discourse in English language education. Nueva York: Routledge.

Garner, Ross. (2017). Insecure positions, heteronomous autonomy and tourism-cultural 
capital: A Bourdieusian reading of tour guides on BBC Worldwide's Doctor Who Experience Walking Tour. Tourist Studies, vol. 17(4), 426-442. http://doi:10.1177/1468797616680851.

Ge, Tianshuang. (2015). The use of modal verbs to express hedging in student academic writing. Research in Corpus Linguistics, vol. 3, 37-46.

Gillaerts, Paul \& Freek Van de Velde. (2010). Interactional Metadiscourse in Research Article Abstracts. Journal of English for Academic Purposes, vol. 9(2), 128-139.

Gillen, Jamie. (2016). Urbanizing existential authenticity: Motorbike tourism in Ho Chi Minh City, Vietnam. Tourist Studies, vol. 16(3), 258-275. http://doi:10.1177/1468797615618035.

Giltrow, Janet. (2005). Modern Conscience: Modalities of Obligation in Research Genres. Text - Interdisciplinary Journal for the Study of Discourse, vol. 25(2), 171-199. http://doi:10.1515/text.2005.25.2.171.

González, Montserrat. (2015). From truth-attesting to intensification: The grammaticalization of Spanish la verdad and Catalan la veritat. Discourse Studies, vol. 17(2), 162-181. http://doi:10.1177/1461445614564519.

Goodwin, Charles. (2006). Retrospective and prospective orientation in the construction of argumentative moves. Text and Talk, vol. 26(4-5), 443-461. http://doi:10.1515/TEXT.2006.018.

Gothie, Sarah Conrad. (2016). Playing "Anne": Red braids, Green Gables, and literary tourists on Prince Edward Island. Tourist Studies, vol. 16(4), 405-421. http://doi:10.1177/1468797615618092.

Gotti, Maurizio \& Marina Dossena. (2001). Introduction. En Maurizio Gotti (Ed.), Modality in Specialized Texts: Selected Papers of the 1st CERLIS Conference, 9-18. Bern, Berlin, Nueva York: Peter Lang.

Greco, Paolo. (2018). Evidentiality and epistemic modality in witness testimony in the context of Italian criminal trials. Journal of Pragmatics, vol. 128, 128-136. https://doi.org/10.1016/i.pragma.2017.10.005.

Haan, Ferdinand de. (1997). The Interaction of Modality and Negation. A Typological Study. Nueva York: Routledge. 
Haan, Ferdinand de. (2008). Typological approaches to modality. En W. Frawley (Ed.), The Expression of Modality, pp. 27-70. Berlin, Nueva York: De Gruyter Mouton. https://doi.org/10.1515/9783110197570.27

Halliday, Michael Alexander K. (1978). Language as social semiotic: the social interpretation of language and meaning. Londres: Edward Arnold.

Halliday, Michael Alexander K., Angus Mclntosh \& Peter Strevens. (1964). The linguistic sciences and language teaching. Londres: Longman.

Halliday, Michael Alexander K. (1985a). Introduction to Functional Grammar. Londres: Edward Arnold.

Halliday, Michael Alexander K. (1985b). Spoken and Written Language. Geelong, Vic.: Deakin University Press.

Halliday, Michael Alexander K. \& Ruqaiya Hasan. (1985). Language, Text and Context. Geelong, Vic.: Deakin University Press.

Halliday, Michael Alexander K. \& Christian Matthias Ingemar Martin Matthiessen. (2013). Halliday's Introduction to Functional Grammar (LSE International Studies). Nueva York: Routledge (Taylor \& Francis Group). https://doi.org/10.4324/9780203431269.

Halliday, Michael Alexander K. \& Christian Matthias Ingemar Martin Matthiessen. (2014). An Introduction to Functional Grammar. Nueva York: Taylor \& Francis.

Haßler, Gerda. (2011). Epistemic modality and evidentiality and their definition on a deictic basis. En Martin G. Becker \& Eva-Maria Remberger (Eds.), Modality and Mood in Romance. Berlin, Nueva York: Walter de Gruyter \& Co. http://doi:10.1515/9783110234343.1.95.

Haugh, Michael, Dániel Z Kádár \& Sara Mills. (2013). Interpersonal pragmatics: Issues and debates. Journal of Pragmatics, vol. 58, 1-11. doi:10.1016/j.pragma.2013.09.009..

Hermann, Inge, Karin Peters \& Emy Van Trijp. (2017). Enrich yourself by helping others: A web content analysis of providers of gap year packages and activities in the Netherlands. Tourist Studies, vol. 17(1), 75-93. http://doi:10.1177/1468797616685649.

Hocking, Bree T. (2016). Gazed and subdued? Spectacle, spatial order and identity in the contested city. Tourist Studies, vol. 16(4), 367-385. 


\section{http://doi:10.1177/1468797615618124.}

Hogeweg, Lotte, Helen de Hoop \& Andrej Malchukov. (2009). The semantics of tense, aspect and modality in the languages of the world. En Lotte Hogeweg, Helen de Hoop \& Andrej L. Malchukov (Eds.) Cross-linguistic Semantics of Tense, Aspect, and Modality, 1-12. Amsterdam, Nueva York: John Benjamins Publishing Company. http://doi:10.1075/la.148.01hog.

Hoye, Leo Francis. (2008). Evidentiality in discourse: A pragmatic and empirical account. En Jesús Romero-Trillo (Ed.), Pragmatics and Corpus Linguistics: A Mutualistic Entente, 151-174. https://doi.org/10.1515/9783110199024.

Hoye, Leo Francis. (2014). Adverbs and modality in English. Londres: Routledge.

Hu, Guangwei \& Feng Cao. (2011). Hedging and boosting in abstracts of applied linguistics articles: A comparative study of English- and Chinese-medium journals. Journal of Pragmatics, vol. 43(11), 2795-2809. http://dx.doi.org/10.1016/i.pragma.2011.04.007.

Huddleston, Rodney. (1984). Introduction to the Grammar of English. Cambridge: Cambridge University Press. http://doi:10.1017/CBO9781139165785.

Huddleston, Rodney \& Geoffrey K. Pullum. (2002). The Cambridge Grammar of the English Language. Cambridge: Cambridge University Press.

Hunston, Susan and Geoff Thompson (eds). (2000). Evaluation in Text. Oxford: Oxford University Press.

Hyland, Ken. (1997). Scientific claims and community values: articulating an academic culture. Language \& Communication, vol. 17(1), 19-31.

Hyland, Ken. (1994). Hedging in academic writing and EAF textbooks. English for Specific Purposes, vol. 13(3), 239-256. http://doi:10.1016/0889-4906(94)90004-3.

Hyland, Ken. (1998). Hedging in Scientific Research Articles (Pragmatics \& Beyond New Series). Vol. 54. Amsterdam: John Benjamins Publishing Company. http://doi:10.1075/pbns.54.

Hyland, Ken. (2001). Bringing in the Reader: Addressee Features in Academic Articles. Written Communication, vol. 18(4), 549-574. 
Hyland, Ken. (2002). Authority and invisibility: Authorial identity in academic writing. Journal of pragmatics, vol. 34, 1091-1112.

Hyland, Ken. (2005a). Stance and engagement: A model of interaction in academic discourse. Discourse Studies, vol. 7(2), 173-192. http://doi:10.1177/1461445605050365.

Hyland, Ken. (2005b). Metadiscourse. Exploring Interaction in Writing. Londres, Nueva York: Continuum.

Hyland, Ken. (2010). Metadiscourse: Mapping Interactions in Academic Writing. Nordic Journal of English Studies, vol. 9(2), 125-143.

Imao, Yasu. (2020). CasualConc. https://sites.google.com/site/casualconc/download.

Jaime, Asunción \& Cristina Pérez-Guillot. (2015). A Comparison Analysis of Modal Auxiliary Verbs in Technical and General English. Procedia - Social and Behavioral Sciences, vol. 212, 292-297. http://doi:10.1016/j.sbspro.2015.11.375.

Kirkham, Sam. (2011). Personal style and epistemic stance in classroom discussion. Language and Literature, vol. 20(3), 201-217. http://doi:10.1177/0963947011413505.

Langacker, Ronald W. (1999). Grammar and Conceptualization. Nueva York: Mouton de Gruyter.

Langacker, Ronald W. (2009). Investigations in Cognitive Grammar. Berlin, Nueva York: Mouton de Gruyter.

Lazard, Gilbert. (2001). On the grammaticalization of evidentiality. Journal of Pragmatics, vol. 33, 359-367. http://doi:10.1016/S0378-2166(00)00008-4.

Leavitt, John, Wallace Chafe \& Johanna Nichols. (1991). Review of Evidentiality: The Linguistic Coding of Epistemology. Language, vol. 67(1), 133-141. http://doi:10.2307/415546.

Lin, Ling \& Stephen Evans. (2012). Structural patterns in empirical research articles: A cross-disciplinary study. English for Specific Purposes, vol. 31(3), 150-160. http://doi:10.1016/j.esp.2011.10.002.

Liston-Heyes, Catherine \& Carol Daley. (2017). Voluntourism, sensemaking and the 
leisure-volunteer duality. Tourist Studies, vol. 17(3), 283-305. http://doi:10.1177/1468797616665769.

Loi, Chek Kim, Jason Miin Hwa Lim \& Sue Wharton. (2016). Expressing an evaluative stance in English and Malay research article conclusions: International publications versus local publications. Journal of English for Academic Purposes,vol. 21, 1-16. http://dx.doi.org/10.1016/j.jeap.2015.08.004.

Lund, Katrín Anna, Kristín Loftsdóttir \& Michael Leonard. (2017). More than a stopover: Analysing the postcolonial image of Iceland as a gateway destination. Tourist Studies, vol. 17(2), 144-163. http://doi:10.1177/1468797616659951.

Lundberg, Christine, Vassilios Ziakas \& Nigel Morgan. (2018). Conceptualising on-screen tourism destination development. Tourist Studies, vol. 18(1), 83-104. http://doi:10.1177/1468797617708511.

Lungo Camiciotti, Gabriella Del. (2008). Two polite speech acts from a diachronic perspective: Aspects of the realisation of requesting and undertaking commitments in the nineteenth-century commercial community. En Aandreas H. Jucker \& Irma Taavitsainen (Eds.), Speech Acts in the History of English, 115-131. Amsterdam/ Nueva York: John Benjamins Publishing. http://doi:10.1075/pbns.176.07del.

Lyons, John. (1977). Semantics. Cambridge: Cambridge University Press.

Lyons, John. (1994). Subjecthood and subjectivity. En Marina Yaguello (Ed.), Subjecthood and Subjectivity: Proceedings of the Colloquium 'The Status of the Subject in Linguistic Theory,' 9-17. Paris: Ophrys.

Manhas, Parikshat Singh. 2016. Climate disparity versus risk management: destination demand analysis. Worldwide Hospitality and Tourism Themes, vol. 8(5), 560-568. http://doi:10.1108/WHATT-06-2016-0037.

Marín-Arrese, Juana I. (2009). Commitment and subjectivity in the discourse of a judicial inquiry. En Raphael Salkie, Pierre Busuttil \& Johan van der Auwera (Eds.), Modality in English. Theory and Description, 237-268. Berlin, Londres: Mouton de Gruyter. https://doi.org/10.1515/9783110213331.237.

Marín Arrese, Juana I. (2009). Effective vs. epistemic stance, and subjectivity/intersubjectivity in political discourse. A case study. En Anastasios 
Tsangalidis \& Roberta Facchinetti (Eds.), Studies on English modality. In honour of Frank R. Palmer, 23-52. Bern, Nueva York: Peter Lang.

Martín-Martín, Pedro. (2008). The Mitigation of Scientific Claims in Research Papers: A Comparitive Study. International Journal of English Studies, vol. 8(2), 133-152. https://doi:10.6018/ijes/8/2/49201.

Martin, James R. (1984). Language, genre and register. En Anne Burns \& Caroline Coffin (eds.), Children Writing: Reader, 21-29. Geelong, Vic.: Deakin University Press.

Martin, James R. \& Peter R. White. (2005). The Language of Evaluation. Londres: Palgrave Macmillan. http://doi:10.1057/9780230511910.

Martin, James R. (2000). Beyond exchange: APPRAISAL systems in English. En Susan Hunston \& Geoffrey Thompson (Eds.), Evaluation in text: authorial stance and the construction of discourse, 142-175. Oxford: Oxford University Press.

Mauranen, Anna. (2018). Second Language Acquisition, world Englishes, and English as a Lingua Franca (ELF). World Englishes, vol. 37(1), 106-119. http://doi:10.1111/weng.12306.

Mendieta-Peñalver, Luis Felipe, José F. Perles-Ribes, Ana B. Ramón-Rodríguez \& María J. Such-Devesa. (2018). Is hotel efficiency necessary for tourism destination competitiveness? An integrated approach. Tourism Economics, vol. 24(1), 3-26. http://doi:10.5367/te.2016.0555.

Moessner, Lilo. (2001). Genre, text type, style, register: A terminological maze? European Journal of English Studies, vol. 5(2), 131-138.

Mongkholjuck, Chinanard. (2008). A Genre Analysis of Tourist Attraction Leaflet Produced and Distributed in Thailand in 2004. Bangkok: Kasetsart University.

Morris, Stephanie \& Scholah Kazi. 2014. Emerging trends regarding accessible accommodation in Dubai luxury hotels. Worldwide Hospitality and Tourism Themes, vol. 6(4), 317-327. http://doi:10.1108/WHATT-01-2014-0004.

Mortelmans, Tanja, Boye, Kasper \& Auwera, Johan. (2009). Modals in the Germanic languages. En Björn Hansen \& Ferdinand de Haan (Eds.), Modals in the Languages of Europe, 11-70. Berlin, Nueva York: De Gruyter Mouton.

https://doi.org/10.1515/9783110219210.1.11 
Moskowich, Isabel \& Begoña Crespo. (2019). "Arguments That Could Possibly Be Urged": Modal Verbs and Tentativeness in the Coruña Corpus. Languages, vol. 4.3(57), 1-12. http://doi:10.3390/languages4030057.

Mur-Dueñas, Pilar. (2011). An Intercultural Analysis of Metadiscourse Features in Research Articles Written in English and in Spanish. Journal of Pragmatics, vol. 43(12), 3068-3079.

Mushin, Ilana. (2013). Making knowledge visible in discourse: Implications for the study of linguistic evidentiality. Discourse Studies, vol. 15(5), 627-645. http://doi:10.1177/1461445613501447.

Narrog, Heiko. (2005). On defining modality again. Language Sciences, vol. 27(2), 165192. http://doi:10.1016/j.langsci.2003.11.007.

Narrog, Heiko. (2012). Modality, Subjectivity, and Semantic Change. Oxford: Oxford University Press. http://doi:10.1093/acprof:oso/9780199694372.001.0001.

Nelson, Kim \& Amie Louise Matthews. (2018). Foreign presents or foreign presence? Resident perceptions of Australian and Chinese tourists in Niseko, Japan. Tourist Studies, vol. 18(2), 213-231. http://doi:10.1177/1468797617717466.

Nuyts, Jan. (2001). Epistemic modality, language, and conceptualization: A cognitivepragmatic perspective. Amsterdam, Nueva York: John Benjamins Publishing Company.

Nuyts, Jan. (2008). Modality: Overview and linguistic issues. The expression of modality. En W. Frawley (Ed.), The Expression of Modality, 1-26. Berlin, Nueva York: De Gruyter Mouton. https://doi.org/10.1515/9783110197570.1

Palmer, Frank R. (1986). Mood and modality. Cambridge: Cambridge University Press.

Palmer, Frank R. (1990). Modality and the English Modals. 2nd edn. Nueva York: Longman.

Palmer, Frank R. (2001). Mood and Modality. Cambridge: Cambridge University Press.

Palmer, Frank R. (2014). Modality and the English modals. Londres: Routledge.

Papafragou, Anna. (1998). Inference and word meaning: The case of modal auxiliaries. Lingua, vol. 105(1-2), 1-47. http://doi:10.1016/s0024-3841(97)00029-6. 
Park, Hyejin, Soobin Seo \& Jay Kandampully. (2016). Why post on social networking sites (SNS)? Examining motives for visiting and sharing pilgrimage experiences on SNS. Journal of Vacation Marketing, vol. 22(4), 307-319. http://doi:10.1177/1356766715615912.

Pic, Elsa \& Gregory Furmaniak. (2012). A study of epistemic modality in academic and popularised discourse: the case of possibility adverbs perhaps, maybe and possibly. Revista de lenguas para fines específicos, vol. 18, 13-44. https://ojsspdc.ulpgc.es/ojs/index.php/LFE/article/view/35.

Plungian, Vladimir A. (2001). The place of evidentiality within the universal grammatical space. Journal of Pragmatics, vol. 18(2), 245-273. http://doi:10.1016/S03782166(00)00006-0.

Portner, Paul. (2009). Modality. Oxford: Oxford University Press.

Reichenberger, Ina. (2017). Why the host community just isn't enough: Processes and impacts of backpacker social interactions. Tourist Studies, vol. 17(3), 263-282. http://doi:10.1177/1468797616665770.

Reitsamer, Bernd Frederik \& Alexandra Brunner-Sperdin. (2017). Tourist destination perception and well-being: What makes a destination attractive? Journal of Vacation Marketing, vol. 23(1), 55-72. http://doi:10.1177/1356766715615914.

Rickly, Jillian M. (2017a). "They all have a different vibe": A rhythmanalysis of climbing mobilities and the Red River Gorge as place. Tourist Studies, vol. 17(3), 223-244. http://doi:10.1177/1468797617717637.

Rickly, Jillian M. (2017b). "I'm a Red River local": Rock climbing mobilities and community hospitalities. Tourist Studies, vol. 17(1), 54-74. http://doi:10.1177/1468797616685648.

Rocci, Andrea. (2017). Modality in Argumentation (Argumentation Library). Vol. 29. Dordrecht: Springer Netherlands. http://doi:10.1007/978-94-024-1063-1. .

Sabila, Nurul Aini Akrima \& Eri Kurniawan. (2020). Move analysis of tourism research article abstracts in national and international journal articles. En Nuria Haristiani, M. Yulianeta, Yanty Wirza, Wawan Gunawan, Eri Kurniawan, Dante Darmawangsa \& Ari Arifin Danuwijaya (Eds.), Proceedings of the 4th International Conference on 
Language, Literature, Culture, and Education (ICOLLITE 2020), 514-520. Paris, France: Atlantis Press.

Saeed, John I. (2016). Semantics. 4th edn. Chichester: Wiley Blackwell.

Salager-Meyer, Françoise. (1992). A text-type and move analysis study of verb tense and modality distribution in medical English abstracts. English for Specific Purposes, vol. 11(2), 93-113.

Salkie, Raphael. (1996). Modality in English and French: A corpus-based approach. Language Sciences, vol. 18(1-2), 381-392.

Salvatierra, Javier \& Gabrielle Walters. (2015). The impact of human-induced environmental destruction on destination image perception and travel behaviour: The case of Australia's Great Barrier Reef. Journal of Vacation Marketing, vol. 23(1), 73-84. http://doi:10.1177/1356766715626966.

Schänzel, Heike A. \& Paul A. Lynch. (2016). Family perspectives on social hospitality dimensions while on holiday. Tourist Studies, vol. 16(2), 133-150. http://doi:10.1177/1468797615594742.

Searle, John R. (1969). Speech Acts: An Essay in the Philosophy of Language. Cambridge: Cambridge University Press.

Shiu, Jerry Yuwen. (2018). Individual rationality and differences in Taiwanese spa hotel choice. Tourism Economics, vol. 24(1), 27-40. http://doi:10.1177/1354816617718972.

Suau-jiménez, Francisca. (2016). What can the discursive construction of stance and engagement voices in traveler forums and tourism promotional websites bring to a cultural, cross-generic and disciplinary view of interpersonality ? Iberica, vol. 31, 199-220.

Suau-Jiménez, Francisca. (2012). El turista 2.0 como receptor de la promoción turística: estrategias lingüísticas e importancia de su estudio. PASOS. Revista de Turismo y Patrimonio Cultural, vol. 10(4), 143-153.

Suau-Jiménez, Francisca \& Rosana Dolón-Herrero. (2007). The importance of metadiscourse in the genre "Promotion of Touristic Services and Products": differences in English and Spanish. En Dita Gálová (Ed.), Languages for specific 
purposes: Searching for common solutions, 71-79. Newcastle upon Tyne: Cambridge Scholars Publishing.

Sulaiman, Mohamed Zain. (2014). Translating the Style of Tourism Promotional Discourse: A Cross Cultural Journey into Stylescapes. Procedia - Social and Behavioral Sciences,vol. 118, 503-510. http://dx.doi.org/10.1016/j.sbspro.2014.02.069.

Swales, John. (1990). Genre Analysis. Cambridge: Cambridge University Press.

Tagliamonte, Sali. (2013). Roots of English: Exploring the History of Dialects. Cambridge: Cambridge University Press.

Taylor, Eunice, Mariam Al Yousuf, Eyad Saleh Nassar, Mohamed Saleh \& Jiji Philip. (2015). The small business dilemma: Understanding and reacting to the unique requirements of Abu Dhabi small businesses in achieving food safety standards. Worldwide Hospitality and Tourism Themes, vol. 7(1), 50-62. https://doi:10.1108/WHATT-12-2014-0040.

Taylor, John R. \& Jeannette Littlemore. (2014). Introduction. En John R. Taylor \& Jeannette Littlemore (Eds.), The Bloomsbury Companion to Cognitive Linguistics, 125. Londres, Nueva York: Bloomsbury Publishing Plc.

Tchoukarine, Igor. (2016). A Place of Your Own on Tito's Adriatic: Club Med and Czechoslovak Trade Union Holiday Resorts in the 1960s. Tourist Studies, vol. 16(4), 386-404. http://doi:10.1177/1468797615618125.

Torabian, Pooneh \& Heather Mair. (2017). (Re)constructing the Canadian border: Antimobilities and tourism. Tourist Studies, vol. 17(1), 17-35. http://doi:10.1177/1468797616685645.

Tribble, Christopher. (2017). ELFA vs. Genre: A new paradigm war in EAP writing instruction? Journal of English for Academic Purposes, vol. 25, 30-44. http://doi:10.1016/j.jeap.2016.10.003.

Tzanelli, Rodanthi \& Maximiliano E. Korstanje. (2016). Tourism in the European economic crisis: Mediatised worldmaking and new tourist imaginaries in Greece. Tourist Studies, vol. 16(3), 296-314. http://doi:10.1177/1468797616648542.

Vanolo, Alberto \& Nadine Cattan. (2017). Selling cruises: Gender and mobility in promotional brochures. Tourist Studies, vol. 17(4), 406-425. 


\section{http://doi:10.1177/1468797616682615.}

Vassileva, Irena. (2001). Commitment and detachment in English and Bulgarian academic writing. English for Specific Purposes, vol. 20(1), 83-102. http://doi:10.1016/S08894906(99)00029-0.

Vendler, Zeno. (1967). Linguistics in Philosophy. Londres: Cornell University Press.

Viechnicki, Gail Brendel. (2002). Evidentiality in scientific discourse. Chicago: University of Chicago.

Walter, Pierre G. (2016). Travelers' experiences of authenticity in "hill tribe" tourism in Northern Thailand. Tourist Studies, vol. 16(2), 213-230. http://doi:10.1177/1468797615594744.

Weatherby, Theodora G. \& Elizabeth S. Vidon. (2018). Delegitimizing wilderness as the man cave: The role of social media in female wilderness empowerment. Tourist Studies, vol. 18(3), 332-352. http://doi:10.1177/1468797618771691.

Werlich, Egon (1976). Genre Analysis. Heidelberg: Quelle and Meyer.

Willett, Thomas. (1988). A Cross-Linguistic Survey of the Grammaticization of Evidentiality. Studies in Language, vol. 12(1), 51-97. http://doi:10.1075/sl.12.1.04wil.

Wright, Georg Henrik von. (1951). An essay in modal logic. Amsterdam: North-Holland.

Yui Ling Ip, Janice. (2008). Analyzing tourism discourse: A case study of a Hong Kong travel brochure. LOCOM Papers, vol. 1, 1-19.

Zerva, Konstantina. (2018). 'Chance Tourism': Lucky enough to have seen what you will never see. Tourist Studies, vol. 18(2), 232-254. http://doi:10.1177/1468797617723471.

Zlatev, Jordan, Timothy P. Racine, Chris Sinha \& Esa Itkonen. (2008). Intersubjectivity. What Makes us Human? En Jordan Zlatev, Timothy P. Racine, Chris Sinha \& Esa Itkonen (Eds.), The Shared Mind Perspectives on intersubjectivity, 1-14. Amsterdam, Philadephia: John Benjamins Publishing Company. 

Apéndice 



\section{Corpus: referencias}

Alnawas, Ibrahim \& Shadi Altarifi. 2016. Exploring the role of brand identification and brand love in generating higher levels of brand loyalty. Journal of Vacation Marketing 22(2). 111-128. http://doi:10.1177/1356766715604663.

Alnawas, Ibrahim \& Shadi Altarifi. 2015. Exploring the role of brand identification and brand love in generating higher levels of brand loyalty. Journal of Vacation Marketing 22(2). 111-128. http://doi:10.1177/1356766715604663.

Anantamongkolkul, Chidchanok, Ken Butcher \& Ying Wang. 2017. The four stages of onsite behavior for a long-stay relaxation holiday. Journal of Vacation Marketing 23(3). 217-232. http://doi:10.1177/1356766716647437.

Andersson, Tommy D., John Armbrecht \& Erik Lundberg. 2017. Linking event quality to economic impact: A study of quality, satisfaction, use value and expenditure at a music festival. Journal of Vacation Marketing 23(2). 114-132. http://doi:10.1177/1356766715615913.

Andersson, Tommy D., Donald Getz, Sanja Vujicic, Richard N.S. Robinson \& Alessio Cavicchi. 2016. Preferred travel experiences of foodies: An application of photo elicitation. Journal of Vacation Marketing 22(1). 55-67. http://doi:10.1177/1356766715589621. 
Aquino, Richard S., Heike A. Schänzel \& Kenneth F. Hyde. 2018. Unearthing the geotourism experience: Geotourist perspectives at Mount Pinatubo, Philippines. Tourist Studies 18(1). 41-62. http://doi:10.1177/1468797617717465.

Atadil, Hilmi A., Ercan Sirakaya-Turk \& Volkan Altintas. 2017. An analysis of destination image for emerging markets of Turkey. Journal of Vacation Marketing 23(1). 3754. http://doi:10.1177/1356766715616858.

Aukland, Knut. 2016. Retailing religion: Guided tours and guide narratives in Hindu pilgrimage. Tourist $\quad$ Studies 16(3). 237-257. http://doi:10.1177/1468797615618038.

Backer, Elisa \& Brian King. 2017. VFR traveller demographics: The social tourism dimension. Journal of Vacation Marketing 23(3). 191-204. http://doi:10.1177/1356766716665439.

Banica, Mirel. 2015. Coach Pilgrimage: Religion, pilgrimage, and tourism in contemporary Romania. Tourist Studies 16(1). 74-87. http://doi:10.1177/1468797616635372.

Barry, Kaya. 2017. Diagramming: A creative methodology for tourist studies. Tourist Studies 17(3). 328-346. http://doi:10.1177/1468797616680852.

Beritelli, Pietro \& Stephan Reinhold. 2018. Chance meetings, the destination paradox, and the social origins of travel: Predicting traveler's whereabouts? Tourist Studies 18(4). 417-441. http://doi:10.1177/1468797617748292.

Bezzola, Toya \& Peter Lugosi. 2018. Negotiating place through food and drink: Experiencing home and away. Tourist Studies 18(4). 486-506. http://doi:10.1177/1468797618791125.

Biraglia, Alessandro, Maximilian H.E.E. Gerrath \& Bryan Usrey. 2018. Examining How Companies' Support of Tourist Attractions Affects Visiting Intentions: The Mediating Role of Perceived Authenticity. Journal of Travel Research 57(6). 811823. http://doi:10.1177/0047287517718352. 
Bolderman, Leonieke \& Stijn Reijnders. 2017. Have you found what you're looking for? Analysing tourist experiences of Wagner's Bayreuth, ABBA's Stockholm and U2's Dublin. Tourist Studies 17(2). 164-181. http://doi:10.1177/1468797616665757.

Bone, Jane \& Kate Bone. 2018. Voluntourism as cartography of self: A Deleuzian analysis of a postgraduate visit to India. Tourist Studies 18(2). 177-193. http://doi:10.1177/1468797617723468.

Bratt, Jonathan. 2018. Return to the east: Tourism promotion as legitimation in Qiandongnan, China. Tourist Studies 18(1). 21-40. http://doi:10.1177/1468797617711575.

Bruttomesso, Elisa. 2018. Making sense of the square: Facing the touristification of public space through playful protest in Barcelona. Tourist Studies 18(4). 467-485. http://doi:10.1177/1468797618775219.

Buckley, Ralf. 2018. Tourism and Natural World Heritage: A Complicated Relationship. Journal of Travel Research 57(5). 563-578. http://doi:10.1177/0047287517713723.

Bui, Huong T. \& Hugh C. Wilkins. 2017. Young Asians' imagination of social distinction. Journal of Vacation Marketing 23(2). 99-113. d http://oi:10.1177/1356766716636927.

Canosa, Antonia, Brent D. Moyle, Char Lee Moyle \& Betty Weiler. 2018. Anthropology and sociology in tourism doctoral research. Tourist Studies 18(4). 375-398. http://doi:10.1177/1468797617737999.

Cashman, David. 2017. 'The most atypical experience of my life': The experience of popular music festivals on cruise ships. Tourist Studies 17(3). 245-262. http://doi:10.1177/1468797616665767.

Chapman, Anya \& Duncan Light. 2017. Working with the carnivalesque at the seaside: Transgression and misbehaviour in a tourism workplace. Tourist Studies 17(2). 182199. http://doi:10.1177/1468797616665768. 
Che-Ha, Norbani, Bang Nguyen, Wan Kalthom Yahya, T. C. Melewar \& Yeo Pei Chen. 2016. Country branding emerging from citizens' emotions and the perceptions of competitive advantage: The case of Malaysia. Journal of Vacation Marketing 22(1). 13-28. http://doi:10.1177/1356766715586454.

Chen, Chun Chu, Wei Jue Huang, Jie Gao \& James F. Petrick. 2018. Antecedents and Consequences of Work-Related Smartphone Use on Vacation: An Exploratory Study of Taiwanese Tourists. Journal of Travel Research 57(6). 743-756. http://doi:10.1177/0047287517714907.

Chen, De Jung. 2018. Couchsurfing: Performing the travel style through hospitality exchange. Tourist Studies 18(1). 105-122. http://doi:10.1177/1468797617710597.

Chen, Qian \& Rong Huang. 2016. Understanding the importance of food tourism to Chongqing, China. Journal of Vacation Marketing 22(1). 42-54. http://doi:10.1177/1356766715589427.

Cheng, Mingming. 2018. A cross-cultural comparison of East and Western academic literature on adventure tourism. Tourist Studies 18(4). 357-374. http://doi:10.1177/1468797617723472.

Cheok, Jason, Torgeir Aleti (né Watne) \& Anne Marie Hede. 2016. Stereotyping Predispositions, activations and applications in cross-cultural service interactions: Views from service providers in Malaysia. Journal of Vacation Marketing 22(2). 98110. http://doi:10.1177/1356766715604661.

Cheok, Jason, Torgeir Aleti (né Watne) \& Anne Marie Hede. 2015. Stereotyping Predispositions, activations and applications in cross-cultural service interactions: Views from service providers in Malaysia. Journal of Vacation Marketing 22(2). 98110. http://doi:10.1177/1356766715604661.

Chung, Namho, Hyunae Lee, Jin Young Kim \& Chulmo Koo. 2018. The Role of Augmented Reality for Experience-Influenced Environments: The Case of Cultural Heritage Tourism in Korea. Journal of Travel Research 57(5). 627-643. http://doi:10.1177/0047287517708255. 
Cooke, Lisa. 2017. Carving "turns" and unsettling the ground under our feet (and skis): A reading of Sun Peaks Resort as a settler colonial moral terrain. Tourist Studies 17(1). 36-53. http://doi:10.1177/1468797616685643.

Correia, Antonia, Metin Kozak \& Seongseop (Sam) Kim. 2018. Luxury shopping orientations of mainland Chinese tourists in Hong Kong: Their shopping destination. Tourism Economics 24(1). 92-108. http://doi:10.1177/1354816617725453.

Country, Bawaka, Sarah Wright, Kate Lloyd, Sandie Suchet-Pearson, Laklak Burarrwanga, Ritjilili Ganambarr, Merrkiyawuy Ganambarr, Banbapuy Ganambarr, Djawundil Maymuru \& Matalena Tofa. 2017. Meaningful tourist transformations with Country at Bawaka, North East Arnhem Land, northern Australia. Tourist Studies 17(4). 443467. http://doi:10.1177/1468797616682134.

Dikgang, Johane \& Edwin Muchapondwa. 2017. The determination of park fees in support of benefit sharing in Southern Africa. Tourism Economics 23(6). 1165-1183. http://doi:10.1177/1354816616655254.

Dumbrăveanu, Daniela, Duncan Light, Craig Young \& Anya Chapman. 2016. Exploring women's employment in tourism under state socialism: Experiences of tourism work in socialist Romania. Tourist Studies 16(2). 151-169. http://doi:10.1177/1468797615594747.

Fernández-Cavia, José, Elena Marchiori, Claire Haven-Tang \& Lorenzo Cantoni. 2017. Online communication in Spanish destination marketing organizations: The view of practitioners. Journal of Vacation Marketing 23(3). 264-273. http://doi:10.1177/1356766716640840.

Fillis, lan, Kim Lehman \& Morgan P. Miles. 2015. The museum of old and new art: Leveraging entrepreneurial marketing to create a unique arts and vacation venture. Journal of Vacation Marketing 23(1). 85-96. http://doi:10.1177/1356766716634153.

Fitzgerald, Jon \& Arianne C. Reis. 2016. Island intersections: Music and tourism on Fernando de Noronha, Brazil. Tourist Studies 16(2). 170-191. http://doi:10.1177/1468797615594738. 
Franklin, Adrian. 2018. Art tourism: A new field for tourist studies. Tourist Studies 18(4). 399-416. http://doi:10.1177/1468797618815025.

Fredman, Peter \& Daniel Wikström. 2018. Income elasticity of demand for tourism at Fulufjället National Park. Tourism Economics 24(1). 51-63. http://doi:10.1177/1354816617724012.

Galloway, Kate. 2018. Curating the aural cultures of the Battery: Soundwalking, auditory tourism and interactive locative media sound art. Tourist Studies 18(4). 442-466. http://doi:10.1177/1468797617723764.

Garcia, Luis Manuel. 2016. Techno-tourism and post-industrial neo-romanticism in Berlin's electronic dance music scenes. Tourist Studies 16(3). 276-295. http://doi:10.1177/1468797615618037.

Garner, Ross. 2017. Insecure positions, heteronomous autonomy and tourism-cultural capital: A Bourdieusian reading of tour guides on BBC Worldwide's Doctor Who Experience Walking Tour. Tourist Studies 17(4). 426-442. http://doi:10.1177/1468797616680851.

Garrigos-Simon, Fernando J., Roberto Llorente, Maria Morant \& Yeamduan Narangajavana. 2016. Pervasive information gathering and data mining for efficient business administration. Journal of Vacation Marketing 22(4). 295-306. http://doi:10.1177/1356766715617219.

Garrigos-Simon, Fernando J., Roberto Llorente, Maria Morant \& Yeamduan Narangajavana. 2016. Pervasive information gathering and data mining for efficient business administration. Journal of Vacation Marketing 22(4). 295-306. http://doi:10.1177/1356766715617219.

Gillen, Jamie. 2016. Urbanizing existential authenticity: Motorbike tourism in Ho Chi Minh City, Vietnam. Tourist Studies 16(3). 258-275. http://doi:10.1177/1468797615618035.

Gothie, Sarah Conrad. 2016. Playing "Anne": Red braids, Green Gables, and literary tourists on Prince Edward Island. Tourist Studies 16(4). 405-421. http://doi:10.1177/1468797615618092. 
Gratch, Ariel. 2018. The angel of living history: Theatricality and representations of the past. Tourist Studies 18(2). 125-141. http://doi:10.1177/1468797617722348.

Grimwood, Bryan S.R. \& Kellee Caton. 2017. Pausing at the intersections of tourism moralities and mobilities: Some neighborhood history and a traffic report. Tourist Studies 17(1). 3-16. http://doi:10.1177/1468797616685640.

Gyimóthy, Szilvia. 2018. Transformations in destination texture: Curry and Bollywood romance in the Swiss Alps. Tourist Studies 18(3). 292-314. http://doi:10.1177/1468797618771692.

Hales, Rob \& Kellee Caton. 2017. Proximity ethics, climate change and the flyer's dilemma: Ethical negotiations of the hypermobile traveller. Tourist Studies 17(1). 94-113. http://doi:10.1177/1468797616685650.

Hermann, Inge, Karin Peters \& Emy Van Trijp. 2017. Enrich yourself by helping others: A web content analysis of providers of gap year packages and activities in the Netherlands. Tourist Studies 17(1). 75-93. http://doi:10.1177/1468797616685649.

Hocking, Bree T. 2016. Gazed and subdued? Spectacle, spatial order and identity in the contested city. Tourist Studies 16(4). 367-385. http://doi:10.1177/1468797615618124.

Huang, Leo \& Michael Chang. 2018. Why do travel agencies choose to undergo IPOs in Taiwan? Tourism Economics 24(1). 79-91. http://doi:10.1177/1354816617725452.

Huang, Songshan (Sam), Amin Afsharifar \& Robert van der Veen. 2016. Examining the moderating role of prior knowledge in the relationship between destination experiences and tourist satisfaction. Journal of Vacation Marketing 22(4). 320-334. d http://oi:10.1177/1356766715618996.

Huang, Songshan (Sam), Amin Afsharifar \& Robert van der Veen. 2016. Examining the moderating role of prior knowledge in the relationship between destination experiences and tourist satisfaction. Journal of Vacation Marketing 22(4). 320-334. http://doi:10.1177/1356766715618996. 
Jarratt, David \& Richard Sharpley. 2017. Tourists at the seaside: Exploring the spiritual dimension. Tourist Studies 17(4). 349-368. http://doi:10.1177/1468797616687560.

Jethro, Duane. 2016. 'Freedom Park, A Heritage Destination': Tour-guiding and visitor experience at a post-apartheid heritage site. Tourist Studies 16(4). 446-461. http://doi:10.1177/1468797615618099.

Jiménez-Esquinas, Guadalupe \& Cristina Sánchez-Carretero. 2018. Who owns the name of a place? On place branding and logics in two villages in Galicia, Spain. Tourist Studies 18(1). 3-20. http://doi:10.1177/1468797617694728.

Jin, Cheng, Jianquan Cheng \& Jing Xu. 2018. Using User-Generated Content to Explore the Temporal Heterogeneity in Tourist Mobility. Journal of Travel Research 57(6). 779791. http://doi:10.1177/0047287517714906.

Johinke, Rebecca. 2018. Take a walk on the wild side: Punk music walking tours in New York City. Tourist Studies 18(3). 315-331. http://doi:10.1177/1468797618771694.

Jönsson, Erik. 2016. The nature of an upscale nature: Bro Hof Slott Golf Club and the political ecology of high-end golf. Tourist Studies 16(3). 315-336. http://doi:10.1177/1468797615618306.

Kang, Eun Jung, Timothy J. Lee \& Ji Sook Han. 2018. The Influence of Enduring Involvement on Tragedy-Related Tourism Experiences. Journal of Travel Research 57(5). 658-670. http://doi:10.1177/0047287517712477.

Kawashima, Tinka Delakorda. 2015. Travel agencies and priests as spiritual leaders: The merits of collaboration. Tourist Studies 16(1). 40-56. http://doi:10.1177/1468797615588430.

Kim, Hyangmi \& Joseph S. Chen. 2016. Destination image formation process: A holistic model. Journal of Vacation Marketing 22(2). 154-166. http://doi:10.1177/1356766715591870. 
Kim, Hyangmi \& Joseph S. Chen. 2015. Destination image formation process: A holistic model. Journal of Vacation Marketing 22(2). 154-166. http://doi:10.1177/1356766715591870.

Lee, Hee Jung \& Hugh Wilkins. 2017. Mass tourists and destination interaction avoidance. Journal of Vacation Marketing 23(1). 3-19. http://doi:10.1177/1356766715617218.

Lee, So Jung, Hak Jun Song, Choong Ki Lee \& James F. Petrick. 2018. An Integrated Model of Pop Culture Fans' Travel Decision-Making Processes. Journal of Travel Research 57(5). 687-701. http://doi:10.1177/0047287517708619.

Lee, Tsung Hung \& Fen Hauh Jan. 2018. Ecotourism Behavior of Nature-Based Tourists: An Integrative Framework. Journal of Travel Research 57(6). 792-810. http://doi:10.1177/0047287517717350.

Li, Jun (Justin), Ip Kin Anthony Wong \& Woo Gon Kim. 2017. Re-segmenting a gaming destination market: A fresh look at Mainland Chinese tourists in Macau. Journal of Vacation Marketing 23(3). 205-216. http://doi:10.1177/1356766716647438.

Liston-Heyes, Catherine \& Carol Daley. 2017. Voluntourism, sensemaking and the leisurevolunteer duality. Tourist Studies 17(3). 283-305. http://doi:10.1177/1468797616665769.

Litvin, Stephen W. \& Wayne W. Smith. 2016. A new perspective on the Plog psychographic system. Journal of Vacation Marketing 22(2). 89-97. http://doi:10.1177/1356766715580187.

Litvin, Stephen W. \& Wayne W. Smith. 2015. A new perspective on the Plog psychographic system. Journal of Vacation Marketing 22(2). 89-97. http://doi:10.1177/1356766715580187.

Lub, Xander D., René Rijnders, Laura Niño Caceres \& Jeroen Bosman. 2016. The future of hotels: The Lifestyle Hub. A design-thinking approach for developing future hospitality concepts. Journal of Vacation Marketing 22(3). 249-264. http://doi:10.1177/1356766715623829. 
Lub, Xander D., René Rijnders, Laura Niño Caceres \& Jeroen Bosman. 2016. The future of hotels: The Lifestyle Hub. A design-thinking approach for developing future hospitality concepts. Journal of Vacation Marketing 22(3). 249-264. http://doi:10.1177/1356766715623829.

Lund, Katrín Anna, Katla Kjartansdóttir \& Kristín Loftsdóttir. 2018. "Puffin love": Performing and creating Arctic landscapes in Iceland through souvenirs. Tourist Studies 18(2). 142-158. http://doi:10.1177/1468797617722353.

Lund, Katrín Anna, Kristín Loftsdóttir \& Michael Leonard. 2017. More than a stopover: Analysing the postcolonial image of Iceland as a gateway destination. Tourist Studies 17(2). 144-163. http://doi:10.1177/1468797616659951.

Lundberg, Christine, Vassilios Ziakas \& Nigel Morgan. 2018. Conceptualising on-screen tourism destination development. Tourist Studies 18(1). 83-104. http://doi:10.1177/1468797617708511.

MacCannell, Dean. 2016. The tourist and the local. Tourist Studies 16(4). 343-350. http://doi:10.1177/1468797615618120.

Manhas, Parikshat Singh. 2016. Climate disparity versus risk management: destination demand analysis. Worldwide Hospitality and Tourism Themes 8(5). 560-568. http://doi:10.1108/WHATT-06-2016-0037.

McWha, Madelene \& Sue Beeton. 2018. Engaging with popular media through tourism: A brief introduction. Tourist Studies 18(3). 257-260. http://doi:10.1177/1468797618782254.

Melissen, Frans, Elena Cavagnaro, Maartje Damen \& Anna Düweke. 2016. Is the hotel industry prepared to face the challenge of sustainable development? Journal of Vacation Marketing 22(3). 227-238. http://doi:10.1177/1356766715618997.

Melissen, Frans, Elena Cavagnaro, Maartje Damen \& Anna Düweke. 2016. Is the hotel industry prepared to face the challenge of sustainable development? Journal of Vacation Marketing 22(3). 227-238. http://doi:10.1177/1356766715618997.

Mendieta-Peñalver, Luis Felipe, José F. Perles-Ribes, Ana B. Ramón-Rodríguez \& María J. Such-Devesa. 2018. Is hotel efficiency necessary for tourism destination 
competitiveness? An integrated approach. Tourism Economics 24(1). 3-26. http://doi:10.5367/te.2016.0555.

Morosan, Cristian. 2018. Information Disclosure to Biometric E-gates: The Roles of Perceived Security, Benefits, and Emotions. Journal of Travel Research 57(5). 644657. http://doi:10.1177/0047287517711256.

Nelson, Kim \& Amie Louise Matthews. 2018. Foreign presents or foreign presence? Resident perceptions of Australian and Chinese tourists in Niseko, Japan. Tourist Studies 18(2). 213-231. http://doi:10.1177/1468797617717466.

Nilsson, Mats \& Mekonnen Tesfahuney. 2018. The post-secular tourist: Re-thinking pilgrimage tourism. Tourist Studies 18(2). 159-176. http://doi:10.1177/1468797617723467.

Oskam, Jeroen \& Tjeerd Zandberg. 2016. Who will sell your rooms? Hotel distribution scenarios. Journal of Vacation Marketing 22(3). 265-278. http://doi:10.1177/1356766715626965.

Oskam, Jeroen \& Tjeerd Zandberg. 2016. Who will sell your rooms? Hotel distribution scenarios. Journal of Vacation Marketing 22(3). 265-278. http://doi:10.1177/1356766715626965.

Pabel, Anja \& Bruce Prideaux. 2016. Social media use in pre-trip planning by tourists visiting a small regional leisure destination. Journal of Vacation Marketing 22(4). 335-348. http://doi:10.1177/1356766715618998.

Pabel, Anja \& Bruce Prideaux. 2016. Social media use in pre-trip planning by tourists visiting a small regional leisure destination. Journal of Vacation Marketing 22(4). 335-348. http://doi:10.1177/1356766715618998.

Park, Hyejin, Soobin Seo \& Jay Kandampully. 2016. Why post on social networking sites (SNS)? Examining motives for visiting and sharing pilgrimage experiences on SNS. Journal of Vacation Marketing 22(4). 307-319. http://doi:10.1177/1356766715615912. 
Park, Hyejin, Soobin Seo \& Jay Kandampully. 2016. Why post on social networking sites (SNS)? Examining motives for visiting and sharing pilgrimage experiences on SNS. Journal of Vacation Marketing 22(4). 307-319. http://doi:10.1177/1356766715615912.

Park, Sun Young \& Michelle Millar. 2016. The US traveler's familiarity with and perceived credibility of lodging ecolabels. Journal of Vacation Marketing 22(1). 3-12. http://doi:10.1177/1356766715585904.

Payne, James E. \& Luis A. Gil-Alana. 2018. Data measurement and the change in persistence of tourist arrivals to the United States in the aftermath of the September 11th terrorist attacks. Tourism Economics 24(1). 41-50. http://doi:10.1177/1354816617719161.

Pearce, Philip L. \& Jarujes Thanksooks. 2016. Towards the future of the domestic hotel in Thailand: A timeline approach. Journal of Vacation Marketing 22(3). 199-211. http://doi:10.1177/1356766715615915.

Pearce, Philip L. \& Jarujes Thanksooks. 2016. Towards the future of the domestic hotel in Thailand: A timeline approach. Journal of Vacation Marketing 22(3). 199-211. http://doi:10.1177/1356766715615915.

Pesonen, Juho Antti \& Anja Tuohino. 2017. Activity-based market segmentation of rural well-being tourists: Comparing online information search. Journal of Vacation Marketing 23(2). 145-158. http://doi:10.1177/1356766715610163.

Peter, Sangeeta \& Victor Anandkumar. 2015. Travel motivation-based typology of tourists who visit a shopping festival: An empirical study on the Dubai shopping festival. Journal of Vacation Marketing 22(2). 142-153. http://doi:10.1177/1356766715607587.

Pham, Tien Duc, Son Nghiem \& Larry Dwyer. 2018. The economic impacts of a changing visa fee for Chinese tourists to Australia. Tourism Economics 24(1). 109-126. http://doi:10.1177/1354816617726204. 
Prince, Solène. 2018. Dwelling in the tourist landscape: Embodiment and everyday life among the craft-artists of Bornholm. Tourist Studies 18(1). 63-82. http://doi:10.1177/1468797617710598.

Raisi, Hossein, Rodolfo Baggio, Llandis Barratt-Pugh \& Gregory Willson. 2018. Hyperlink Network Analysis of a Tourism Destination. Journal of Travel Research 57(5). 671686. http://doi:10.1177/0047287517708256.

Reichenberger, Ina. 2017. Why the host community just isn't enough: Processes and impacts of backpacker social interactions. Tourist Studies 17(3). 263-282. http://doi:10.1177/1468797616665770.

Reitsamer, Bernd Frederik \& Alexandra Brunner-Sperdin. 2017. Tourist destination perception and well-being: What makes a destination attractive? Journal of Vacation Marketing 23(1). 55-72. http://doi:10.1177/1356766715615914.

Richard, Brendan \& Shane Cleveland. 2016. The future of hotel chains: Branded marketplaces driven by the sharing economy. Journal of Vacation Marketing 22(3). 239-248. http://doi:10.1177/1356766715623827.

Richard, Brendan \& Shane Cleveland. 2016. The future of hotel chains: Branded marketplaces driven by the sharing economy. Journal of Vacation Marketing 22(3). 239-248. http://doi:10.1177/1356766715623827.

Rickly, Jillian M. 2017. "I'm a Red River local": Rock climbing mobilities and community hospitalities. Tourist Studies 17(1). 54-74. http://doi:10.1177/1468797616685648.

Rickly, Jillian M. 2017. "They all have a different vibe": A rhythmanalysis of climbing mobilities and the Red River Gorge as place. Tourist Studies 17(3). 223-244. http://doi:10.1177/1468797617717637.

Rodríguez, Andrea Martín \& John F. O’Connell. 2018. Can low-cost long-haul carriers replace Charter airlines in the long-haul market? A European perspective. Tourism Economics 24(1). 64-78. http://doi:10.1177/1354816617724017. 
Rojas-Méndez, José I. \& Michael J. Hine. 2017. Countries' positioning on personality traits: Analysis of 10 South American national tourism websites. Journal of Vacation Marketing 23(3). 233-247. http://doi:10.1177/1356766716649227.

Ryan, Louise. 2016. Re-branding Tasmania: MONA and the altering of local reputation and identity. Tourist Studies 16(4). 422-445. http://doi:10.1177/1468797615618097.

Sahli, Alia Besbes \& Patrick Legohérel. 2016. The tourism Web acceptance model: A study of intention to book tourism products online. Journal of Vacation Marketing 22(2). 179-194. http://doi:10.1177/1356766715607589.

Sahli, Alia Besbes \& Patrick Legohérel. 2015. The tourism Web acceptance model: A study of intention to book tourism products online. Journal of Vacation Marketing 22(2). 179-194. http://doi:10.1177/1356766715607589.

Salvatierra, Javier \& Gabrielle Walters. 2015. The impact of human-induced environmental destruction on destination image perception and travel behaviour: The case of Australia's Great Barrier Reef. Journal of Vacation Marketing 23(1). 7384. http://doi:10.1177/1356766715626966.

Sampaio, Sofia. 2017. Tourism, gender and consumer culture in late- and postauthoritarian Portugal. Tourist Studies 17(2). 200-217. http://doi:10.1177/1468797616665771.

Schäfer, Stefanie. 2016. From Geisha girls to the Atomic Bomb Dome: Dark tourism and the formation of Hiroshima memory. Tourist Studies 16(4). 351-366. http://doi:10.1177/1468797615618122.

Schänzel, Heike A. \& Paul A. Lynch. 2016. Family perspectives on social hospitality dimensions while on holiday. Tourist Studies 16(2). 133-150. http://doi:10.1177/1468797615594742.

Šerić, Maja, Irene Gil-Saura \& Josip Mikulić. 2017. Customer-based brand equity building: Empirical evidence from Croatian upscale hotels. Journal of Vacation Marketing 23(2). 133-144. http://doi:10.1177/1356766716634151. 
Shiu, Jerry Yuwen. 2018. Individual rationality and differences in Taiwanese spa hotel choice. Tourism Economics 24(1). 27-40. http://doi:10.1177/1354816617718972.

Skinner, Jonathan. 2015. Walking the Falls: Dark tourism and the significance of movement on the political tour of West Belfast. Tourist Studies 16(1). 23-39. http://doi:10.1177/1468797615588427.

So, Kevin Kam Fung, Laurie Wu, Lina Xiong \& Ceridwyn King. 2018. Brand Management in the Era of Social Media: Social Visibility of Consumption and Customer Brand Identification. Journal of Travel Research 57(6). 727-742. http://doi:10.1177/0047287517718354.

Solnet, David, Tom Baum, Richard N.S. Robinson \& Leonie Lockstone-Binney. 2016. What about the workers? Roles and skills for employees in hotels of the future. Journal of Vacation Marketing 22(3). 212-226. http://doi:10.1177/1356766715617403.

Solnet, David, Tom Baum, Richard N.S. Robinson \& Leonie Lockstone-Binney. 2016. What about the workers? Roles and skills for employees in hotels of the future. Journal of Vacation Marketing 22(3). 212-226. http://doi:10.1177/1356766715617403.

Song, Hanqun. 2016. Theatrical performance in the tourism industry: An importancesatisfaction analysis. Journal of Vacation Marketing 22(2). 129-141. http://doi:10.1177/1356766715604664.

Song, Hanqun. 2015. Theatrical performance in the tourism industry: An importancesatisfaction analysis. Journal of Vacation Marketing 22(2). 129-141. http://doi:10.1177/1356766715604664.

Song, Myungkeun, Breffni M. Noone \& Anna S. Mattila. 2018. A Tale of Two Cultures: Consumer Reactance and Willingness to Book Fenced Rates. Journal of Travel Research 57(6). 707-726. http://doi:10.1177/0047287517713722.

Straub, Leslie Ellen. 2015. Negotiation and experience: Space and place in religious pilgrimage. Tourist Studies 16(1). 88-104. http://doi:10.1177/1468797616635378.

Sun, Sunny, Rob Law \& Tony Tse. 2016. Exploring price fluctuations across different online travel agencies: A case study of room reservations in an upscale hotel in Hong Kong. 
Journal of Vacation Marketing 22(2). 167-178. http://doi:10.1177/1356766715592663.

Sun, Sunny, Rob Law \& Tony Tse. 2015. Exploring price fluctuations across different online travel agencies: A case study of room reservations in an upscale hotel in Hong Kong. Journal of Vacation Marketing 22(2). 167-178. http://doi:10.1177/1356766715592663.

Sun, Sunny, Karen Tsz Lun Tong \& Rob Law. 2017. Chinese hotel guest perception of international chain hotels under the same hotel brand in different travel destinations: The cases of intercontinental and Sheraton. Journal of Vacation Marketing 23(2). 172-188. http://doi:10.1177/1356766715614344.

Tasci, Asli D.A. \& Yong Jae Ko. 2017. Travel needs revisited. Journal of Vacation Marketing 23(1). 20-36. http://doi:10.1177/1356766715617499.

Tchoukarine, Igor. 2016. A Place of Your Own on Tito's Adriatic: Club Med and Czechoslovak Trade Union Holiday Resorts in the 1960s. Tourist Studies 16(4). 386404. http://doi:10.1177/1468797615618125.

Tegtmeyer, Lina L. 2016. Tourism aesthetics in ruinscapes: Bargaining cultural and monetary values of Detroit's negative image. Tourist Studies 16(4). 462-477. http://doi:10.1177/1468797615618100.

Torabian, Pooneh \& Heather Mair. 2017. (Re)constructing the Canadian border: Antimobilities and tourism. Tourist Studies 17(1). 17-35. http://doi:10.1177/1468797616685645.

Torres, Edwin N. \& Marissa Orlowski. 2017. Let's 'Meetup' at the theme park. Journal of Vacation Marketing 23(2). 159-171. http://doi:10.1177/1356766716634152.

Tuominen, Pasi P. \& Mário P. Ascenção. 2016. The hotel of tomorrow: A service design approach. Journal of Vacation Marketing 22(3). 279-292. http://doi:10.1177/1356766716637102. 
Tuominen, Pasi P. \& Mário P. Ascenção. 2016. The hotel of tomorrow: A service design approach. Journal of Vacation Marketing 22(3). 279-292. http://doi:10.1177/1356766716637102.

Tussyadiah, lis P., Timothy Hyungsoo Jung \& M. Claudia tom Dieck. 2018. Embodiment of Wearable Augmented Reality Technology in Tourism Experiences. Journal of Travel Research 57(5). 597-611. http://doi:10.1177/0047287517709090.

Tzanelli, Rodanthi \& Maximiliano E. Korstanje. 2016. Tourism in the European economic crisis: Mediatised worldmaking and new tourist imaginaries in Greece. Tourist Studies 16(3). 296-314. http://doi:10.1177/1468797616648542.

Valenta, Marko \& Zan Strabac. 2015. The dramaturgical nexus of ethno-religious, tourist and transnational frames of pilgrimages in post-conflict societies: The Bosnian and Herzegovinian experience. Tourist Studies 16(1). 57-73. http://doi:10.1177/1468797616635371.

Nuenen, Tom van. 2016. Here I am: Authenticity and self-branding on travel blogs. Tourist Studies 16(2). 192-212. http://doi:10.1177/1468797615594748.

Vanolo, Alberto \& Nadine Cattan. 2017. Selling cruises: Gender and mobility in promotional brochures. Tourist Studies 17(4). 406-425. http://doi:10.1177/1468797616682615.

Vorobjovas-Pinta, Oskaras \& Brady Robards. 2017. The shared oasis: An insider ethnographic account of a gay resort. Tourist Studies 17(4). 369-387. http://doi:10.1177/1468797616687561.

Walter, Pierre G. 2016. Travelers' experiences of authenticity in "hill tribe" tourism in Northern Thailand. Tourist Studies 16(2). 213-230. http://doi:10.1177/1468797615594744.

Wang, Li \& Pertti Alasuutari. 2017. Co-construction of the tourist experience in social networking sites: Two forms of authenticity intertwined. Tourist Studies 17(4). 388405. http://doi:10.1177/1468797616687559. 
Wang, Yuan, Xiang (Robert) Li \& Kun Lai. 2018. A Meeting of the Minds: Exploring the Core-Periphery Structure and Retrieval Paths of Destination Image Using Social Network Analysis. Journal of Travel Research 57(5). 612-626. http://doi:10.1177/0047287517706262.

Weatherby, Theodora G. \& Elizabeth S. Vidon. 2018. Delegitimizing wilderness as the man cave: The role of social media in female wilderness empowerment. Tourist Studies 18(3). 332-352. http://doi:10.1177/1468797618771691.

Weaver, David, Chuanzhong Tang, Fangfang Shi, Ming Feng Huang, Kevin Burns \& Ang Sheng. 2018. Dark Tourism, Emotions, and Postexperience Visitor Effects in a Sensitive Geopolitical Context: A Chinese Case Study. Journal of Travel Research 57(6). 824-838. http://doi:10.1177/0047287517720119.

White, Leanne. 2018. Qantas still calls Australia home: The spirit of Australia and the flying kangaroo. Tourist Studies 18(3). 261-274. http://doi:10.1177/1468797618785617.

Xue, Lan \& Deborah Kerstetter. 2018. Discourse and Power Relations in Community Tourism. Journal of Travel Research 57(6). 757-768. http://doi:10.1177/0047287517714908.

Yeoman, Ian, Jeroen Oskam \& Albert Postma. 2016. The future of hotels: Vacation marketing, service design and management. Journal of Vacation Marketing 22(3). 197-198. http://doi:10.1177/1356766716649720.

Yeoman, Ian, Jeroen Oskam \& Albert Postma. 2016. The future of hotels: Vacation marketing, service design and management. Journal of Vacation Marketing 22(3). 197-198. http://doi:10.1177/1356766716649720.

Yousaf, Salman \& Nida Samreen. 2016. Information agents and cultural differences as determinants of country's reputation and its subsequent effects on tourism prospects of a country in sustained crises: The case of Pakistan. Journal of Vacation Marketing 22(4). 365-384. http://doi:10.1177/1356766715623828.

Yousaf, Salman \& Nida Samreen. 2016. Information agents and cultural differences as determinants of country's reputation and its subsequent effects on tourism 
prospects of a country in sustained crises: The case of Pakistan. Journal of Vacation Marketing 22(4). 365-384. http://doi:10.1177/1356766715623828.

Yu, Xi, Gerardo Joel Anaya, Li Miao, Xinran Lehto \& Ip Kin Anthony Wong. 2018. The Impact of Smartphones on the Family Vacation Experience. Journal of Travel Research 57(5). 579-596. http://doi:10.1177/0047287517706263.

Zaidan, Esmat. 2017. Analysis of ICT usage patterns, benefits and barriers in tourism SMEs in the Middle Eastern countries: The case of Dubai in UAE. Journal of Vacation Marketing 23(3). 248-263. http://doi:10.1177/1356766716654515.

Zaidan, Esmat A. 2016. Tourism shopping and new urban entertainment: A case study of Dubai. Journal of Vacation Marketing 22(1). 29-41. http://doi:10.1177/1356766715589426.

Zavattaro, Staci M. \& Joshua J. Daspit. 2016. A grounded theoretical approach to understanding innovation in destination marketing organizations. Journal of Vacation Marketing 22(4). 349-364. http://doi:10.1177/1356766715623826.

Zerva, Konstantina. 2018. 'Chance Tourism': Lucky enough to have seen what you will never see. Tourist Studies 18(2). 232-254. http://doi:10.1177/1468797617723471.

Zhang, Jingru, Ying Gui, Bihu Wu, Alastair M. Morrison \& Cong Li. 2016. Is destination marketing organization microblogging in China delivering? An empirical analysis of information supply against consumer information needs. Journal of Vacation Marketing 22(1). 68-85. http://doi:10.1177/1356766715591869.

Zhang, Xiaoyu \& Chris Ryan. 2018. Investigating tourists' and local residents' perceptions of a Chinese film site. Tourist Studies 18(3). 275-291. http://doi:10.1177/1468797618771693. 